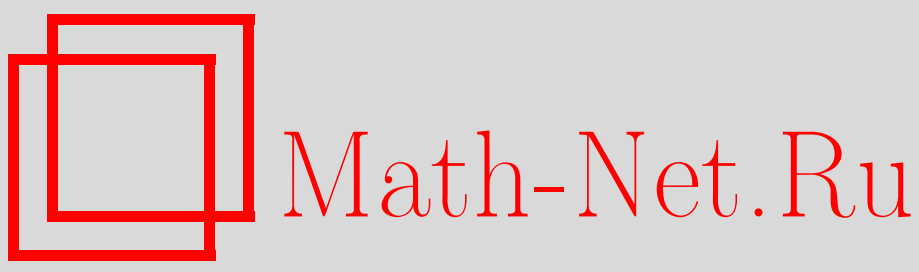

И. А. Чельцов, Двойное пространство с двойной прямой, Матем. сб., 2004, том 195, номер 10, 109-156

DOI: https://doi.org/10.4213/sm855

Использование Общероссийского математического портала Math-Net.Ru подразумевает, что вы прочитали и согласны с пользовательским соглашением

http: //www. mathnet.ru/rus/agreement

Параметры загрузки:

IP : 3.89 .185 .249

26 апреля 2023 г., $17: 08: 38$ 
УДК 512.7

\author{
И.А. Чельцов
}

\title{
Двойное пространство с двойной прямой
}

\begin{abstract}
В работе классифицируются бирациональные перестройки двойного накрытия $\mathbb{P}^{3}$ с ветвлением в поверхности степени шесть, имеющей двойную особенность вдоль прямой, в трехмерные многообразия Фано с каноническими особенностями, расслоения на эллиптические кривые и расслоения на поверхности кодаировой размерности нуль.

Библиография: 22 названия.
\end{abstract}

\section{§1. Введение}

Многообразия ${ }^{1}$, обладающие обильным антиканоническим дивизором, называются многообразиями Фано (см. [1]), а в двумерном случае-поверхностями дель Пецио. Важность многообразий Фано во многом обусловлена программой минимальных моделей, из которой следует, что с точки зрения бирациональной геометрии многообразия Фано являются строительными блоками многообразий, имеющих отрицательную кодаирову размерность. В размерности два и три все неособые многообразия Фано классифицированы, а их бирациональная геометрия хорошо изучена. В частности, все поверхности дель Пещо рациональны. Большинство неособых трехмерных многообразий Фано также рациональны. С другой стороны, существуют нерациональные трехмерные многообразия Фано, например неособая трехмерная кубика или квартика.

Куб антиканонического дивизора трехмерного многообразия Фано, обычно называемый степенъю многообразия Фано, является его основным бирегулярньм инвариантом, который во многом определяет бирациональную геометрию многообразия Фано. Например, из классификации следует, что все трехмерные неособые многообразия Фано степени не меньше 26 рациональны. И наоборот, при уменьшении степени бирациональная геометрия трехмерных многообразий Фано становится более жесткой. Известно, что степень неособого трехмерного многообразия Фано всегда четна и, в частности, не может быть менњше двух. Более того, сушествует всего одно неособое трехмерное многообразие Фано степени два - двойное накрытие $\mathbb{P}^{3}$ с ветвлением в неособой поверхности степени шесть. Бирациональная геометрия последнего многообразия хорошо изучена. В частности, показано, что двойное накрытие $\mathbb{P}^{3}$ с ветвлением в неособой секстике нерационально и не может быть бирационально перестроено в расслоение на поверхности с отрицательной кодаировой размерностью.

\footnotetext{
${ }^{1}$ Все многообразия считаются проективньми, нормальньми и определенными надполем $\mathbb{C}$.
} 
Хорошо известно, что в отличие от бирациональной теории алгебраических поверхностей в трехмерном случае необходимо рассматривать уже многообразия с особенностями, так как класса неособых многообразий не хватает для построения правильной бирациональной теории многомерных алгебраических многообразий. Таким образом, возникает естественньй вопрос изучения бирациональной геометрии особых трехмерных многообразий Фано. Однако множество примеров показывает, что последняя задача может быть очень сложна даже в простейших случаях. Следовательно, естественно попытаться понять досконально бирациональную геометрию двойного накрытия $\mathbb{P}^{3}$ с ветвлением в особой секстике, что поможет хотя бы прояснить ситуацию в общем случае.

Рассмотрим двойное накрытие $\gamma: V \rightarrow \mathbb{P}^{3}$ с ветвлением в секстике $S \subset \mathbb{P}^{3}$ такое, что поверхность $S$ неособа вне некоторой прямой $L \subset S$ и поверхность $S$ имеет особенность типа $x^{2}+y^{2}=0 \subset \mathbb{C}^{3}$ в общей точке прямой $L$. Многообразие $V$ неособо вне собственного прообраза $\widetilde{L} \subset V$ прямой $L$ и $V$ имеет особенность типа $\mathbb{A}_{1} \times \mathbb{C}$ в общей точке кривой $\widetilde{L}$. Как легко видеть, многообразие $V$ может быть задано уравнением

$$
u^{2}=x^{2} p_{4}(x, y, z, t)+y^{2} q_{4}(x, y, z, t)
$$

во взвешенном проективном пространстве $\mathbb{P}(1,1,1,3)$, где $p_{4}$ и $q_{4}$ - однородные многочлены степени четыре, $x, y, z$ и $t$ - однородные координаты веса один и $u$ - однородная координата веса три. Пучок плоскостей в $\mathbb{P}^{3}$, проходящих через прямую $L$, задает пучок поверхностей $\mathscr{P}$ на многообразии $V$ такой, что нормализация общей поверхности в пучке $\mathscr{P}$ является гладкой поверхностью дель Пеццо степени два. Рассмотрим ограничение $f: X \rightarrow V$ на многообразие $V$ раздутия гладкой кривой $\widetilde{L} \subset \mathbb{P}(1,1,1,3)$.

ЗАмечАниЕ 1.1. В дальнейшем будет предполагаться, что многообразие $X$ гладкое, а уравнения $p_{4}(0,0, z, t)=q_{4}(0,0, z, t)=0$ имеют ровно восемь различных однородных решений, которые задают восемь различных точек $O_{i} \in \widetilde{L}$ для $i=1, \ldots, 8$.

Несложно видеть, что многообразие $X$ может быть задано как двулистное накрытие трехмерного многообразия $\operatorname{Proj}\left(\mathscr{O}_{\mathbb{P}^{1}} \oplus \mathscr{O}_{\mathbb{P}^{1}} \oplus \mathscr{O}_{\mathbb{P}^{1}}(1)\right)$ с ветвлением в поверхности $R_{X} \sim 4 M+2 H$, где $M$ и $H$ - тавтологический пучок и слой проекции на $\mathbb{P}^{1}$ многообразия $\operatorname{Proj}\left(\mathscr{O}_{\mathbb{P}^{1}} \oplus \mathscr{O}_{\mathbb{P}^{1}} \oplus \mathscr{O}_{\mathbb{P}^{1}}(1)\right)$ соответственно. В частности, из неособости $X$ следует неособость поверхности $R_{X}$. С другой стороны, поверхность $R_{X}$ является обильным дивизором на многообразии $\operatorname{Proj}\left(\mathscr{O}_{\mathbb{P}^{1}} \oplus \mathscr{O}_{\mathbb{P}^{1}} \oplus \mathscr{O}_{\mathbb{P}^{1}}(1)\right)$. Последнее вместе с теоремой Лефшеца влекут $\operatorname{Pic}(X)=\mathbb{Z} \oplus \mathbb{Z}$. Таким образом, $V-$ многообразие $\Phi$ ано с $\mathbb{Q}$-факториальными каноническими особенностями и группой Пикара $\mathbb{Z}$, дивизор $K_{V}$ является дивизором Картье, $-K_{V}^{3}=2$, линейная система $\left|-K_{V}\right|$ свободна, $\varphi_{\left|-K_{V}\right|}=\gamma, K_{X}=f^{*}\left(K_{V}\right)$, пучок $\left|-K_{X}-E\right|$ задает расслоение $\tau: X \rightarrow \mathbb{P}^{1}$ на поверхности дель Пещю степени два, $f$ имеет восемь приводимых слоев $Z_{i}=Z_{i}^{0} \cup Z_{i}^{1}$ над точками $O_{i}$, где $E-f$-исключительный дивизор, кривые $Z_{i}^{0}$ и $Z_{i}^{1}$ неособы и пересекаются трансверсально в одной точке.

ЗАмечАниЕ 1.2 . Последовательное раздутие кривой $Z_{i}^{k}$ и стандартный антифлип в собственном прообразе кривой $Z_{i}^{1-k}$ задают бирациональную перестройку $V$ в многообразие с численно эффективным и объемным антиканоническим 
классом, которое определяет бирациональную перестройку $\rho_{i, j}: V \rightarrow V_{i, k}$, где $V_{i, k}$ - каноническое многообразие Фано с $-K_{V_{i, k}}^{3}=\frac{1}{2}($ см. $\S 4)$.

Основная цель настоящей работы состоит в доказательстве следующих результатов.

ТЕОРема 1.3. Пусть $\rho: V \rightarrow \mathbb{P}^{2}$ - доминантное рациональное отображение, чей общий слой является эллиптической кривой. Тогда существует рациональное отображение $\alpha: \mathbb{P}^{2} \rightarrow \mathbb{P}^{1}$ такое, что $\alpha \circ \rho=\tau \circ f^{-1}$.

Теорема 1.4. Многообразие Фано $V$ не мохет бъть бирационально перестроено ни в какое многообразие Фано с каноническими особенностями, кроме самого многообразия $V$ и шестнадиати многообразий Фано $V_{i, k}$ для $i=1, \ldots, 8 u k=0,1$.

Следует отметить, что утверждение теоремы 1.3 неможет быть улучшено во всех возможных случаях. Действительно, рассмотрим произвольное сечение $C$ расслоения $\tau$ и линейную подсистему $\mathscr{H}_{C} \subset\left|-K_{X}+\tau^{*}\left(\mathscr{O}_{\mathbb{P}^{1}}(n)\right)\right|$ для $n \gg 0$, состоящую из поверхностей, проходящих через кривую $C$. В этом случае несложно показать, что общий слой рационального отображения $\varphi \mathscr{H}_{C}: X--\rightarrow Z_{C}$ является эллиптической кривой, поверхность $Z_{C}$ рациональна, разрешения неопределенностей рационального отображения $\varphi \mathscr{H}_{C}$ задает бирациональную перестройку многообразия $X$ в эллиптическое расслоение с сечением и существует естественное отображение $\alpha_{C}: Z_{C} \rightarrow \mathbb{P}^{1}$ такое, что $\alpha_{C} \circ \varphi \mathscr{H}_{C}=\tau$. С другой стороны, в [1] была доказана

Теорема 1.5. Пусть $Y$ - проективное многообразие, $g: Y \rightarrow R$ - обладающий сечением сюрвективный морфизм на гладкую кривую $R, r_{1}, \ldots, r_{k} \in R-$ замкнутые точки такие, что слои $Y_{i}=g^{-1}\left(r_{i}\right)$ неособы и сепарабельно раиионально связны, и пусть $y_{i} \in Y_{i}$ - произвольные замкнутые точки. Тогда существует сечение $C \subset Y$ морфизма $g$ такое, что $y_{i} \in C, i=1, \ldots, k$.

Как показывает следующий результат (см. [2]), расслоение $\tau$ всегда имеет сечение.

ТЕОРема 1.6. Пусть $Y$ - гладкая собственная и геометрически неприводимая поверхность над $C_{1}$-полем $\mathbb{F}$ такая, что $Y$ рачиональна над алгебраическим замыканием поля $\mathbb{F}$. Тогда $Y$ имеет точку, определенную над полем $\mathbb{F}$.

Значит, множество сечений расслоения $\tau: X \rightarrow \mathbb{P}^{1}$ очень велико. Более того, используя технику настояшей работы, можно показать, что для двух достаточно общих сечений $C_{1}$ и $C_{2}$ расслоения $\tau$ рациональные отображения $\varphi \mathscr{H}_{C_{1}}$ и $\varphi \mathscr{H}_{C_{2}}$ задают неэквивалентные ${ }^{2}$ эллиптические расслоения. Таким образом, утверждение теоремы 1.3 не может быть улучшено, однако оно может быть дополнено посредством следующего результата работы [3], поскольку структура групп бирациональных автоморфизмов поверхностей дель Пеццо степени два была описана в работах [4], [5].

\footnotetext{
${ }^{2}$ Расслоения $\tau: U \rightarrow Z$ и $\bar{\tau}: \bar{U} \rightarrow \bar{Z}$ считаются әквивалентными в случае существования двух бирациональных отображений $\alpha: U \rightarrow \bar{U}$ и $\beta: Z \rightarrow \bar{Z}$ таких, что $\bar{\tau} \circ \alpha=\beta \circ \tau$, а $\alpha$ индуцирует изоморфизм общих слоев расслоений $\tau$ и $\bar{\tau}$.
} 
Теорема 1.7. Пусть $Y$ - гладкая поверхность дель Пецио степени два, определенная над полем $\mathbb{F}$, выполнено условие $\operatorname{Pic}(Y) \cong \mathbb{Z}, \rho: Y \rightarrow U-$ бирациональное отображение, $a \omega: U \rightarrow R$ - эллиптическое расслоение. Тогда существует бирациональный автоморфизм $\sigma$ поверхности $Y$ и бирациональный морфизм $\alpha: W \rightarrow Y$ такие, что $K_{W}^{2}=0$, дивизор $-K_{W}$ численно эффективен, для некоторого $n \in \mathbb{N}$ линейная система $\left|-n K_{W}\right|$ свободна, морфизм $\varphi_{\left|-n K_{W}\right|}$ является әллиптическим расслоением, а расслоение $\varphi_{\left|-n K_{W}\right|} \circ \rho^{-1} \circ \sigma \circ \rho$ әквивалентно расслоению $\omega$.

Бирациональные перестройки алгебраических многообразий в расслоения на эллиптические кривые использовались в работах [6], [7] для доказательства потенциальной плотности ${ }^{3}$ рациональных точек на гладких трехмерных многообразиях Фано, где был получен следующий результат.

Теорема 1.8. Рациональные точки потенциально плотны на всех гладких трехмерных многообразиях Фано, за исключением, быть может, семейства гладких двойных накрытий $\mathbb{P}^{3}$, разветвленных в гладкой поверхности степени шесть.

Возможное исключение в утверждении теоремы 1.8 обусловлено следуюшим результатом работы [3].

ТЕОРема 1.9. Пусть $Y$ - двойное накрытие $\mathbb{P}^{3}$, разветвленное в гладкой секстике. Тогда $Y$ не может быть бирационально перестроено в расслоения на әллиптические кривье, многообразие $Y$ бирачионально не изоморфно никакому трехмерному многообразию Фано с каноническими особенностями, кроме самого себя, все бирациональнье перестройки многообразия $Y$ в расслоения на поверхности кодаировой размерности нуль индуцированы пучками в линейной системе $\left|-K_{Y}\right|$.

Отметим, что из классификации гладких трехмерных многообразий Фано и теоремы 1.9 легко следует тот факт, что двойное накрытие $\mathbb{P}^{3}$, разветвленное в гладкой секстике, является единственным гладким многообразием Фано, которое не может быть бирационально перестроено в эллиптическое расслоение. Много примеров многомерных рационально связных многообразий, которые не могут быть бирационально перестроены в эллиптические расслоения, приведено в работах [3] и [8]-[10]. Следующий результат получен в работе [11].

Теорема 1.10. Пусть $\theta: Y \rightarrow \mathbb{P}^{3}$ - двойное накрытие с ветвлением в поверхности степени шесть $S_{Y} \subset \mathbb{P}^{3}$ такое, что $S_{Y}$ неособа всюду, за исключением одной точки $O \in S_{Y}$, в которой $S_{Y}$ имеет особенность типа $\mathbb{A}_{1}$. Тогда $Y$ бирачионально не изоморфно никакому многообразию Фано с каноническими особенностями, кроме самого себя, все бирачиональнье перестройки $Y$ в расслоения на поверхности кодаировой размерности нуль заданы пучками

\footnotetext{
${ }^{3}$ Рациональные точки многообразия $X$, определенного над числовьм полем $\mathbb{F}$, потенциально плотны, если существует такое конечное расширение полей $\mathbb{K} / \mathbb{F}$, что множество $\mathbb{K}$-рациональных точек многообразия $X(\mathbb{K})$ плотно в топологии Зариского.
} 
в $\left|-K_{Y}\right|$, многообразие $Y$ бирационально изоморфно единственному әллиптическому расслоению, которое индуцировано проекцией из точки О.

Многообразие $V$ изучалось в работе [12], где был получен следующий результат.

ТеОрема 1.11. Многообразие V бирационально не изоморфно никакому расслоению на рациональные поверхности, которое не эквивалентно расслоению $\tau: X \rightarrow \mathbb{P}^{1}$, последовательность групл $1 \rightarrow \Gamma \rightarrow \operatorname{Bir}(V) \rightarrow \mathbb{G} \rightarrow 1$ точна, әде $\Gamma$ - свободное произведение инволючий Бертини общего слоя расслоения $\tau$, который рассматривается как гладкая поверхность дель Пецио степени два c группой Пикара $\mathbb{Z}$, определенная над полем $\mathbb{C}(x)$, а группа $\mathbb{G}$ является группой послойных бирациональных автоморфизмов расслоения $\tau$, индуцирующих изоморфизм общего слоя расслоения $\tau$.

В частности, $V$ нерационально. Следующий результат был получен в работе [13].

ТЕОрема 1.12. Предположим, что кажддй слой $F$ расслоения $\tau$ неособ вдоль каждой неприводимой кривой $C \subset F$ такой, что кривая $\gamma \circ f(C)$ является прямой и отображение $\left.\gamma \circ f\right|_{C}$ является изоморфизмом. Тогда трехмерное многообразие $V$ бирационально не изоморфно никакому трехмерному многообразию Фано с терминальными $\mathbb{Q}$-факториальными особенностями и группой Пикара $\mathbb{Z}$, последовательность групп $1 \rightarrow \Gamma \rightarrow \operatorname{Bir}(V) \rightarrow \operatorname{Aut}(V) \rightarrow 1$ точна, где Г - свободное произведение инволющий Бертини общего слоя расслоения $\tau$, который рассматривается как гладкая поверхность дель Пецио степени два с әруппой Пикара $\mathbb{Z}$, определенная над $\mathbb{C}(x)$.

Следующий результат был получен в работе [14].

Tеорема 1.13. Пусть $\tau_{1}: X_{1} \rightarrow \mathbb{P}^{1}$ и $\tau_{2}: X_{2} \rightarrow \mathbb{P}^{1}$ - расслоения на поверхности дель Пеццо степени два, $\alpha: X_{1}-\rightarrow X_{2}$ и $\beta: \mathbb{P}^{1} \rightarrow \mathbb{P}^{1}$ - бирациональные отображения такие, что $\beta \circ \tau_{1}=\tau_{2} \circ \alpha$, бирациональное отображсение $\alpha$ индуиирует изоморфизм общих слоев расслоений $\tau_{1} u \tau_{2}$, оба многообразия $X_{1}$ и $X_{2}$ неособы. Тогда бирациональное отображение $\alpha$ является изоморфизмом.

СледСТВИЕ 1.14. Последовательность групп $1 \rightarrow \Gamma \rightarrow \operatorname{Bir}(V) \rightarrow \operatorname{Aut}(V) \rightarrow 1$ точна, әде группа Г - свободное произведение инволюиий Бертини общего слоя расслоения $\tau$, который рассматривается как гладкая поверхность дель Пецио степени два с группой Пикара $\mathbb{Z}$, определенная над полем $\mathbb{C}(x)$.

В настоящей работе следствие 1.14 будет независимо получено в $\S 6$, а также будет доказана

ТЕОРЕМА 1.15. Предположим, что исключительная поверхность $E \subset X$ является неособой, а прямая $L$ является единственной прямой на секстике $S$, проходящей через точки $\gamma\left(O_{i}\right) \in \mathbb{P}^{3}$. Пусть $\rho: V \rightarrow \mathbb{P}^{1}$ - рациональное отображсние, чей общий слой является неприводимой поверхностью кодаировой размерности нуль. Тогда существует бирациональный автоморфизм б

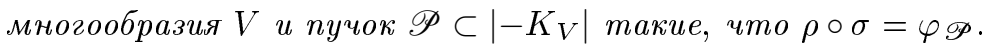


В доказательствах теорем 1.3, 1.4 и 1.15 будет использован так называемый метод супер-максимальной особенности из работы [15].

ЗАмечание 1.16. Линейная система $\left|-(k+1) K_{X}-k E\right|$ свободна для $k \gg 0$ и задает двойное накрытие многообразия $\operatorname{Proj}\left(\mathscr{O}_{\mathbb{P}^{1}} \oplus \mathscr{O}_{\mathbb{P}^{1}} \oplus \mathscr{O}_{\mathbb{P}^{1}}(1)\right)$, разветвленное в поверхности $R_{X} \sim 4 M+2 H$, где $M$ и $H$ - тавтологический пучок и слой проекции на $\mathbb{P}^{1}$ многообразия $\operatorname{Proj}\left(\mathscr{O}_{\mathbb{P}^{1}} \oplus \mathscr{O}_{\mathbb{P}^{1}} \oplus \mathscr{O}_{\mathbb{P}^{1}}(1)\right)$ соответственно.

ПримеР 1.17. Гиперповерхность $u^{2}=x^{2}\left(x^{4}+z^{4}+t^{4}\right)+y^{2}\left(y^{4}+z^{4}+2 t^{4}\right)$ в $\mathbb{P}(1,1,1,3)$ удовлетворяет всем условиям теорем $1.3,1.4$ и 1.15 , где $x, y, z$ и $t-$ однородные координаты веса один, а $u$-однородная координата веса три.

Автор признателен А.А. Борисову, Л. Воцлаву, М.М. Гриненко, В.А. Исковских, А. Корти, М. Мелле, Дж. Парку, Ю.Г. Прохорову, А.В. Пухликову и В.В. Шокурову за плодотворные беседы. Автор выражает большую благодарность рецензенту за ряд полезных замечаний.

\section{§2. Подвижные лог-пары}

В настоящем параграфе будут рассмотрены свойства подвижных лог-пар, которые были введены в работе [16].

ОПРЕДЕЛЕНИЕ 2.1. Под подвижной лог-парой $\left(X, M_{X}\right)$ подразумевается многообразие $X$ вместе с подвижной границей $M_{X}$, где $M_{X}=\sum_{i=1}^{n} a_{i} \mathscr{M}_{i}$ - формальная конечная линейная комбинация линейных систем $\mathscr{M}_{i}$ на $X$ без неподвижных компонент такая, что $a_{i} \in \mathbb{Q} \geqslant 0$.

Подвижная лог-пара может быть рассмотрена как обычная лог-пара путем замены каждой линейной системы либо на ее общий элемент, либо на взвешенную сумму ее общих элементов. В частности, для данной подвижной лог-пары $\left(X, M_{X}\right)$ подвижная граница $M_{X}$ может быть рассмотрена как эффективный дивизор, а $K_{X}+M_{X}$ будет называться лог-каноническим дивизором подвижной лог-пары $\left(X, M_{X}\right)$. В оставшейся части этого параграфа лог-канонические дивизоры всех рассматриваемых лог-пар будут считаться дивизорами $\mathbb{Q}$-Картье.

ЗАмечАниЕ 2.2. Для подвижной лог-пары $\left(X, M_{X}\right)$ самопересечение $M_{X}^{2}$ может быть рассмотрено как корректно определенный эффективный цикл коразмерности два на многообразии $X$, если многообразие $X$ имеет $\mathbb{Q}$-факториальные особенности.

В отличие от обычных лог-пар прямой образ подвижной границы при бирациональном отображении естественно и корректно определен.

ОПРЕДЕЛЕНИЕ 2.3. Подвижные лог-пары $\left(X, M_{X}\right)$ и $\left(Y, M_{Y}\right)$ назьваются $\sigma u$ рационально эквивалентными, если существует бирациональное отображение $\rho: X \rightarrow Y$ такое, что $M_{Y}=\rho\left(M_{X}\right)$.

Дискрепантности, терминальность, каноничность, лог-терминальность и логканоничность могут быть определены для подвижных лог-пар аналогично тому, как они определяются для обычных лог-пар (см. [17]). 
ЗАмечАниЕ 2.4. Применение лог-программы минимальњых моделей к каноничным и терминальным подвижньм лог-парам сохраняет их каноничность и терминальность соответственно.

Каждая подвижная лог-пара бирационально эквивалентна подвижной лог-паре с каноническими особенностями, и особенности подвижной лог-пары совпадают с особенностями многообразия вне объединения базисных множеств компонент подвижной границы.

ОПРЕДЕЛЕНИЕ 2.5. Собственное неприводимое подмногообразие $Y \subset X$ называется чентром канонических особенностей подвижсной лог-пары $\left(X, M_{X}\right)$, если сушествуют бирациональный морфизм $f: W \rightarrow X$ и $f$-исключительный дивизор $E_{1} \subset W$ такие, что

$$
K_{W}+f^{-1}\left(M_{X}\right) \sim_{\mathbb{Q}} f^{*}\left(K_{X}+M_{X}\right)+\sum_{i=1}^{k} a\left(X, M_{X}, E_{i}\right) E_{i},
$$

вьполнено $a\left(X, M_{X}, E_{1}\right) \leqslant 0$ и $f\left(E_{1}\right)=Y$, где $a\left(X, M_{X}, E_{i}\right) \in \mathbb{Q}$, а дивизор $E_{i}$ является $f$-исключительным.

ОПРЕДЕЛЕниЕ 2.6. Через $\mathbb{C} \mathbb{S}\left(X, M_{X}\right)$ будем обозначать множество всех центров канонических особенностей подвижной лог-пары $\left(X, M_{X}\right)$, а через $\operatorname{CS}\left(X, M_{X}\right)$ будем обозначать объединение всех центров канонических особенностей подвижной лог-пары $\left(X, M_{X}\right)$, рассматриваемое как собственное подмножество многообразия $X$.

В частности, подвижная лог-пара $\left(X, M_{X}\right)$ терминальна $\Longleftrightarrow \mathbb{C} S\left(X, M_{X}\right)=\varnothing$.

ОПРЕДЕЛЕНИЕ 2.7. Собственное неприводимое подмногообразие $Y \subset X$ называется собственным иентром канонических особенностей подвижной лог$n a p ы ~\left(X, M_{X}\right)$, если существуют такой бирациональный морфизм $f: W \rightarrow X$ и такой $f$-исключительный дивизор $E_{1} \subset W$, что

$$
K_{W}+f^{-1}\left(M_{X}\right) \sim_{\mathbb{Q}} f^{*}\left(K_{X}+M_{X}\right)+\sum_{i=1}^{k} a\left(X, M_{X}, E_{i}\right) E_{i},
$$

вьполнено $a\left(X, M_{X}, E_{1}\right) \leqslant 0$ и $f\left(E_{1}\right)=Y$, подмногообразие $Y \subset X$ содержится в базисном множестве по крайней мере одной компоненты подвижной границы $M_{X}$, где $a\left(X, M_{X}, E_{i}\right) \in \mathbb{Q}$, а дивизор $E_{i}$ является $f$-исключительным.

ОПРЕДЕЛЕНИЕ 2.8. Через $\overline{\mathbb{C S}}\left(X, M_{X}\right)$ будем обозначать множество всех собственных центров канонических особенностей подвижной лог-пары $\left(X, M_{X}\right)$, а через $\overline{\mathrm{CS}}\left(X, M_{X}\right)$ будем обозначать объединение всех собственных центров канонических особенностей лог-пары $\left(X, M_{X}\right)$, рассматриваемое как подмножество многообразия $X$.

ЗАмечание 2.9. Справедливо соотношение

$$
\mathbb{C S}\left(X, M_{X}\right)=\overline{\mathbb{C S}}\left(X, M_{X}\right) \cup \mathbb{C S}(X, \varnothing),
$$

в частности, в случае, когда многообразие $X$ имеет лиш терминальные особенности, $\mathbb{C S}\left(X, M_{X}\right)=\overline{\mathbb{C S}}\left(X, M_{X}\right)$. 
ОПРЕДЕЛЕНИЕ 2.10. Особенности $\left(X, M_{X}\right)$ полутерминальнь, если

$$
\overline{\mathbb{C S}}\left(X, M_{X}\right)=\varnothing \text {. }
$$

ЗАмЕчАнИЕ 2.11. Если особенности лог-пары $\left(X, M_{X}\right)$ полутерминальны, то для достаточно малого $\varepsilon \in \mathbb{Q}_{>1}$ особенности лог-пары $\left(X, \varepsilon M_{X}\right)$ также полутерминальны.

ОПРЕДЕЛЕНИЕ 2.12. Число

$$
\varkappa\left(X, M_{X}\right)= \begin{cases}\operatorname{dim}\left(\varphi_{\left|n m\left(K_{W}+M_{W}\right)\right|}(W)\right) & \text { для таких } n \gg 0, \\ & \text { что }\left|n\left(K_{W}+M_{W}\right)\right| \neq \varnothing ; \\ -\infty, & \text { если }\left|n m\left(K_{W}+M_{W}\right)\right|=\varnothing \\ & \text { для всех натуральных чисел } n,\end{cases}
$$

называется размерностью Кодаиры подвижной лог-пары $\left(X, M_{X}\right)$, где подвижная лог-пара $\left(W, M_{W}\right)$ бирационально эквивалентна лог-паре $\left(X, M_{X}\right)$ и имеет канонические особенности, а $m \in \mathbb{N}$ такое, что дивизор $m\left(K_{W}+M_{W}\right)$ является дивизором Картье.

Лемма 2.13. Размерность Кодаиры подвижной лог-пары корректно определена и не зависит от выбора бирационально эквивалентной подвижной лог-пары с каноническими особенностями.

ДоКАЗАТЕЛЬСтво. Предположим, что две подвижные лог-пары $\left(X, M_{X}\right)$ и $\left(Y, M_{Y}\right)$ имеют канонические особенности и $M_{X}=\rho\left(M_{Y}\right)$ для некоторого бирационального отображения $\rho: Y \rightarrow X$. Рассмотрим натуральные числа $m_{X}$ и $m_{Y}$ такие, что оба дивизора $m\left(K_{X}+M_{X}\right)$ и $m\left(K_{Y}+M_{Y}\right)$ являются дивизорами Картье. Для доказательства необходимого утверждения достаточно показать, что либо обе линейные системы $\left|n m_{X}\left(K_{X}+M_{X}\right)\right|$ и $\left|n m_{Y}\left(K_{Y}+M_{Y}\right)\right|$ пусты для всех натуральных $n$, либо

$$
\varphi_{\left|n m_{X}\left(K_{X}+M_{X}\right)\right|}(X)=\varphi_{\left|n m_{Y}\left(K_{Y}+M_{Y}\right)\right|}(Y)
$$

для достаточно больших натуральных чисел $n$. Рассмотрим бирациональные морфизмы $g: W \rightarrow X$ и $f: W \rightarrow Y$ такие, что $W$ неособо и $\rho=g \circ f^{-1}$. Тогда

$$
K_{W}+M_{W} \sim_{\mathbb{Q}} g^{*}\left(K_{X}+M_{X}\right)+\Sigma_{X} \sim_{\mathbb{Q}} f^{*}\left(K_{Y}+M_{Y}\right)+\Sigma_{Y},
$$

где $M_{W}=g^{-1}\left(M_{X}\right)$, а $\Sigma_{X}$ и $\Sigma_{Y}$ - исключительные дивизоры бирациональных морфизмов $g$ и $f$ соответственно. Каноничность лог-пар $\left(X, M_{X}\right)$ и $\left(Y, M_{Y}\right)$ влечет эффективность дивизоров $\Sigma_{X}$ и $\Sigma_{Y}$. Для достаточно большого и достаточно делимого натурального числа $k$ из эффективности дивизоров $\Sigma_{X}$ и $\Sigma_{Y}$ следует, что размерность линейных систем $\left|k\left(K_{W}+M_{W}\right)\right|,\left|g^{*}\left(k\left(K_{X}+M_{X}\right)\right)\right|$ и $\left|f^{*}\left(k\left(K_{Y}+M_{Y}\right)\right)\right|$ одинакова и

$$
\varphi_{\left|k\left(K_{W}+M_{W}\right)\right|}=\varphi_{\left|g^{*}\left(k\left(K_{X}+M_{X}\right)\right)\right|}=\varphi_{\left|f^{*}\left(k\left(K_{Y}+M_{Y}\right)\right)\right|}
$$

в случае, когда они непусты, откуда следует требуемое утверждение.

По определению размерность Кодаиры подвижной лог-пары является бирациональным инвариантом и неубывающей функцией от коэффициентов границы. 
ОПРЕДЕЛЕНИЕ 2.14. Подвижная лог-пара $\left(V, M_{V}\right)$ называется канонической моделью подвижной лог-пары $\left(X, M_{X}\right)$, если существует отображение $\psi: X \rightarrow V$ такое, что $\psi$ бирационально, $M_{V}=\psi\left(M_{X}\right)$, лог-канонический дивизор $K_{V}+M_{V}$ обилен, а особенности лог-пары $\left(V, M_{V}\right)$ каноничны.

ТЕорема 2.15. Каноническая модель единственна, если существует.

ДокАЗАТЕльСтво. Предположим, что подвижные лог-пары $\left(X, M_{X}\right)$ и $\left(V, M_{V}\right)$ являются каноническими моделями и $M_{X}=\rho\left(M_{V}\right)$ для некоторого бирационального отображения $\rho: V \rightarrow X$. Пусть $g: W \rightarrow X$ и $f: W \rightarrow V-$ бирациональные отображения такие, что $\rho=g \circ f^{-1}$. Тогда выполнено соотношение

$$
K_{W}+M_{W} \sim_{\mathbb{Q}} g^{*}\left(K_{X}+M_{X}\right)+\Sigma_{X} \sim_{\mathbb{Q}} f^{*}\left(K_{V}+M_{V}\right)+\Sigma_{V}
$$

где $M_{W}=g^{-1}\left(M_{X}\right)=f^{-1}\left(M_{V}\right)$ и дивизоры $\Sigma_{X}$ и $\Sigma_{V}$ являются исключительными дивизорами бирациональных морфизмов $g$ и $f$ соответственно. Из каноничности особенностей лог-пар $\left(X, M_{X}\right)$ и $\left(V, M_{V}\right)$ следует, что оба дивизора $\Sigma_{X}$ и $\Sigma_{V}$ эффективны. Рассмотрим достаточно большое и делимое натуральное число $n$ такое, что все три дивизора $n\left(K_{W}+M_{W}\right), n\left(K_{X}+M_{X}\right)$ и $n\left(K_{V}+M_{V}\right)$ одновременно являются дивизорами Картье. Из эффективности дивизоров $\Sigma_{X}$ и $\Sigma_{V}$ следует, что

$$
\varphi_{\left|n\left(K_{W}+M_{W}\right)\right|}=\varphi_{\left|g^{*}\left(n\left(K_{X}+M_{X}\right)\right)\right|}=\varphi_{\left|f^{*}\left(n\left(K_{V}+M_{V}\right)\right)\right|}
$$

и $\rho$ является изоморфизмом, поскольку дивизоры $K_{X}+M_{X}$ и $K_{V}+M_{V}$ обильны.

Отметим, что из сушествования канонической модели подвижной лог-пары следует, что ее размерность Кодаиры равна размерности многообразия.

\section{§3. Предварительные результаты}

Как уже упоминалось в предыдушем параграфе, подвижные границы могут рассматриваться как эффективные дивизоры, а подвижные лог-пары могут рассматриваться как обычные лог-пары. Таким образом, можно рассматривать и лог-пары, имеющие как подвижные, так и неподвижные компоненты. В данном параграфе не будут налагаться никакие ограничения на коэффициенты границ, в частности, границы могут не быть эффективными, если это не оговорено особо, однако будет подразумеваться, что все лог-канонические дивизоры являются $\mathbb{Q}$-Картье дивизорами.

ОПРЕДЕЛЕНИЕ 3.1. Для лог-пары $\left(X, B_{X}\right)$ и бирационального морфизма $f: V \rightarrow X$ лог-пара $\left(V, B^{V}\right)$ называется лог-поднятием лог-парь $\left(X, B_{X}\right)$, если вьполнены соотношения

$$
B^{V}=f^{-1}\left(B_{X}\right)-\sum_{i=1}^{n} a\left(X, B_{X}, E_{i}\right) E_{i}
$$

и

$$
K_{V}+B^{V} \sim_{\mathbb{Q}} f^{*}\left(K_{X}+B_{X}\right)
$$

такие, что $a\left(X, B_{X}, E_{i}\right) \in \mathbb{Q}$ и дивизор $E_{i}$ является $f$-исключительным. 
ОПРЕДЕЛЕНИЕ 3.2. Собственное неприводимое подмногообразие $Y \subset X$ называется иентром лог-канонических особенностей лог-пары $\left(X, B_{X}\right)$, если сушествуют бирациональный морфизм $f: W \rightarrow X$ и дивизор $E \subset W$ такие, что дивизор $E$ содержится в носителе эффективной части дивизора $\left\lfloor B^{Y}\right\rfloor$.

ОПРЕДЕЛЕНИЕ 3.3. Через $\mathbb{L} \mathbb{C} S\left(X, B_{X}\right)$ будем обозначать множество всех центров лог-канонических особенностей лог-пары $\left(X, B_{X}\right)$, а через $\operatorname{LCS}\left(X, B_{X}\right)$ будем обозначать объединение всех центров лог-канонических особенностей лог-пары $\left(X, B_{X}\right)$, рассматриваемое как собственное подмножество многообразия $X$.

Рассмотрим лог-пару $\left(X, B_{X}\right)$, где $B_{X}=\sum_{i=1}^{k} a_{i} B_{i}$, дивизор $B_{i}$ эффективен и неприводим и $a_{i} \in \mathbb{Q}$. Возьмем бирациональный морфизм $f: Y \rightarrow X$ такой, что $Y$ гладкое, а объединение всех дивизоров $f^{-1}\left(B_{i}\right)$ и всех $f$-исключительных дивизоров образует дивизор с простьми нормальными пересечениями. Морфизм $f$ называется лог-разрешением лог-nары $\left(X, B_{X}\right)$, и выполнено

$$
K_{Y}+B^{Y} \sim_{\mathbb{Q}} f^{*}\left(K_{X}+B_{X}\right)
$$

для лог-поднятия $\left(Y, B^{Y}\right)$ лог-пары $\left(X, B_{X}\right)$.

ОПРЕДЕЛЕНИЕ 3.4. Подсхемой лоъ-канонических особенностей лог-парь $\left(X, B_{X}\right)$ называется подсхема, ассоциированная с пучком идеалов $\mathscr{I}\left(X, B_{X}\right)=$ $f_{*}\left(\left\lceil-B^{Y}\right\rceil\right)$, которая будет обозначаться $\mathscr{L}\left(X, B_{X}\right)$.

Носитель подсхемы $\mathscr{L}\left(X, B_{X}\right)$ совпадает с множеством $\operatorname{LCS}\left(X, B_{X}\right) \subset X$. Следующий результат является теоремой В.В. Шокурова об обращении в нуль.

Теорема 3.5. Пусть $\left(X, B_{X}\right)$ - лог-пара, граница $B_{X}$ әффективна, а $H$ численно эффективный и обвемный дивизор на $X$ такой, что $D=K_{X}+$ $B_{X}+H$ является дивизором Картье. Тогда $H^{i}\left(X, \mathscr{I}\left(X, B_{X}\right) \otimes D\right)=0$ для вcex $i>0$.

ДоказАтЕЛьство. По относительной теореме Каваматы-Фивега об обращении в нуль $R^{i} f_{*}\left(f^{*}\left(K_{X}+B_{X}+H\right)+\left\lceil-B^{W}\right\rceil\right)=0$ для всех $i>0$ (см. [17]). Из вырождения соответствуюшей спектральной последовательности и

$$
R^{0} f_{*}\left(f^{*}\left(K_{X}+B_{X}+H\right)+\left\lceil-B^{W}\right\rceil\right)=\mathscr{I}\left(X, B_{X}\right) \otimes D
$$

следует, что для всех $i \geqslant 0$

$$
H^{i}\left(X, \mathscr{I}\left(X, B_{X}\right) \otimes D\right)=H^{i}\left(W, f^{*}\left(K_{X}+B_{X}+H\right)+\left\lceil-B^{W}\right\rceil\right),
$$

но для $i>0$

$$
H^{i}\left(W, f^{*}\left(K_{X}+B_{X}+H\right)+\left\lceil-B^{W}\right\rceil\right)=0
$$

по теореме Каваматы-Фивега об обращении в нуль.

Для дивизора Картье $D$ на $X$ рассмотрим точную последовательность

$$
0 \rightarrow \mathscr{I}\left(X, B_{X}\right) \otimes D \rightarrow \mathscr{O}_{X}(D) \rightarrow \mathscr{O}_{\mathscr{L}\left(X, B_{X}\right)}(D) \rightarrow 0
$$

из теоремы 3.5 вытекают следуюшие две теоремы о связности В. В. Шокурова. 
Теорема 3.6. Пусть $\left(X, B_{X}\right)$ - лог-пара, $B_{X}$ эффективна, $-\left(K_{X}+B_{X}\right)$ численно әффективен и обгемен. Тогда $\operatorname{LCS}\left(X, B_{X}\right)$ связно.

Tеорема 3.7. Пусть $\left(X, B_{X}\right)$ - лог-пара, $B_{X}$ әффективна, $-\left(K_{X}+B_{X}\right)$ численно әффективен и обвемен относительно некоторого морфизма $g: X \rightarrow Z$ со связными слоями. Тогда $\operatorname{LCS}\left(X, B_{X}\right)$ связно в окрестности каждого слоя морфизма $g$.

Следующий результат является теоремой 17.4 работы [18].

ТеОрема 3.8. Пусть $g: X \rightarrow Z-м о р ф и з \mathcal{M}, D_{X}=\sum_{i \in I} d_{i} D_{i}-$ дивизор на многообразии $X, h: V \rightarrow X$ - разрешение особенностей $X$ такие, что $g_{*}\left(\mathscr{O}_{X}\right)=\mathscr{O}_{Z}$, $и я и з о р-\left(K_{X}+D_{X}\right)$ численно әффективен и обгемен относительно морфизма $g$, коразмерность каждого подмногообразия $g\left(D_{i}\right) \subset Z$ больше либо равна 2 в случае $d_{i}<0$, обвединение всех дивизоров $h^{-1}\left(D_{i}\right)$ и всех -исключительных дивизоров образует дивизор с простыми нормальными пересечениями. Тогда множество $\bigcup_{a_{E} \leqslant-1} E$ связно в окрестности каждого слоя морфизма $g \circ h$, где рациональные числа $a_{E}$ заданы посредством $\mathbb{Q}$-рациональной эквивалентности $K_{V} \sim_{\mathbb{Q}} f^{*}\left(K_{X}+D_{X}\right)+\sum_{E \subset V} a_{E} E$. Тогда

ДокАЗАТЕЛЬство. Положим $f=g \circ h, A=\sum_{a_{E}>-1} E$ и $B=\sum_{a_{E} \leqslant-1} E$.

$$
\lceil A\rceil-\lfloor B\rfloor \sim_{\mathbb{Q}} K_{V}-h^{*}\left(K_{X}+D_{X}\right)+\{-A\}+\{B\}
$$

и $R^{1} f_{*} \mathscr{O}_{V}(\lceil A\rceil-\lfloor B\rfloor)=0$ по относительной теореме Каваматы-Фивега об обрашении в нуль (см. [17]). Таким образом, отображение $f_{*} \mathscr{O}_{V}(\lceil A\rceil) \rightarrow f_{*} \mathscr{O}_{\lfloor B\rfloor}(\lceil A\rceil)$ сюръективно, но каждая неприводимая компонента дивизора $\lceil A\rceil$ либо $h$-исключительна, либо является собственным прообразом некоторого дивизора $D_{j}$ с $d_{j}<0$. Следовательно, дивизор $h_{*}(\lceil A\rceil)$ является $g$-исключительным и

$$
f_{*} \mathscr{O}_{V}(\lceil A\rceil)=\mathscr{O}_{Z}
$$

Значит, отображение $\mathscr{O}_{Z} \rightarrow f_{*} \mathscr{O}_{\lfloor B\rfloor}(\lceil A\rceil)$ сюръективно, откуда следует связность $\lfloor B\rfloor$ в окрестности слоев морфизма $f$, поскольку $\lceil A\rceil$ эффективен и не имеет общих компонент с $\lfloor B\rfloor$.

В предыдущем параграфе в определениях 2.5 и 2.6 были определены центр канонических особенностей подвижной лог-пары и нескольких связанных с ним объектов, в которых подвижность границы нигде реально не использовалась. Однако в данном случае такое излишшее предположение, как подвижность, все-таки оправдано тем, что введенные понятия используются в основном для подвижных лог-пар и возникают в некоторых естественных конструкциях, связанных в основном с подвижньми лог-парами. Тем не менее иногда эти понятия удобно использовать и для обыкновенных лог-пар, как правило, для индуктивной связи с их лог-аналогами. Причем, как уже отмечалось, такое использование вполне корректно. 
Tеорема 3.9. Пусть $\left(X, B_{X}\right)$ - лог-пара, $B_{X}$ әффективна, $Z \in \mathbb{C}\left(X, B_{X}\right)$, дивизор $H$ является әффективным и неприводимым дивизором Картве на $X$ таким, что $Z \subset H$, дивизор $H$ не является компонентой границы $B_{X}$, подмногообразие $H$ гладко в общей точке многообразия $Z$. Тогда

$$
Z \in \mathbb{L} \mathbb{C S}\left(H,\left.B_{X}\right|_{H}\right)
$$

ДоказАТЕЛЬСТво. Пусть $f: W \rightarrow X$-лог-разрешение лог-пары $\left(X, B_{X}+H\right)$, положим $\widehat{H}=f^{-1}(H)$. Тогда выполнено соотношение

$$
K_{W}+\widehat{H} \sim_{\mathbb{Q}} f^{*}\left(K_{X}+B_{X}+H\right)+\sum_{E \neq \widehat{H}} a\left(X, B_{X}+H, E\right) E
$$

и по предположению $\{Z, H\} \subset \operatorname{LCS}\left(X, B_{X}+H\right)$. Из применения теоремы 3.8 к лог-поднятию лог-пары $\left(X, B_{X}+H\right)$ на $W$ следует, что $\widehat{H} \cap E \neq \varnothing$ для некоторого $f$-исключительного дивизора $E$ на $W$ с $f(E)=Z$ и $a\left(X, B_{X}, E\right) \leqslant-1$. Из эквивалентности

$$
\left.\left.K_{\widehat{H}} \sim\left(K_{W}+\widehat{H}\right)\right|_{\widehat{H}} \sim_{\mathbb{Q}} f\right|_{\widehat{H}} ^{*}\left(K_{H}+\left.B_{X}\right|_{H}\right)+\left.\sum_{E \neq \hat{H}} a\left(X, B_{X}+H, E\right) E\right|_{\widehat{H}}
$$

следует необходимое утверждение.

Следуюший результат является теоремой 3.1 работы [19], его доказательство из работы [19] будет приведено практически без изменений, поскольку на сегодняшний день оно является самым простым способом доказательства очень важного трехмерного следствия, которое будет приведено ниже.

Теорема 3.10. Пусть $H$ - поверхность, $O$ - гладкая точка на $H, M_{H}-$ әффективная подвижная граница на поверхности $H, a_{1}$ и $a_{2}$ - неотрицательные рачиональные числа, $\Delta_{1}$ и $\Delta_{2}$ - неприводимье и приведенные кривые на $H$, которье пересекаются нормально в точке $O$. Тогда из $O \in$ $\mathbb{L} \mathbb{C S}\left(H,\left(1-a_{1}\right) \Delta_{1}+\left(1-a_{2}\right) \Delta_{2}+M_{H}\right)$ cледует

$$
\operatorname{mult}_{O}\left(M_{H}^{2}\right) \geqslant\left\{\begin{array}{lll}
4 a_{1} a_{2} & \text { в случае } a_{1} \leqslant 1 \text { или } a_{2} \leqslant 1 \\
4\left(a_{1}+a_{2}-1\right) & \text { в случае } a_{1}>1 \text { и } a_{2}>1,
\end{array}\right.
$$

и неравенство строгое, если $\left(H,\left(1-a_{1}\right) \Delta_{1}+\left(1-a_{2}\right) \Delta_{2}+M_{H}\right)$ не лог-канонична в $O$.

ДоказАтельство. Пусть $D=\left(1-a_{1}\right) \Delta_{1}+\left(1-a_{2}\right) \Delta_{2}+M_{H}$. Рассмотрим бирациональный морфизм $f: S \rightarrow H$ такой, что поверхность $S$ гладкая и вьполнено

$$
K_{S}+f^{-1}(D) \sim_{\mathbb{Q}} f^{*}\left(K_{H}+D\right)+\sum_{i=1}^{k} a\left(H, D, E_{i}\right) E_{i}
$$

где $E_{i}-f$-исключительная кривая, $a\left(H, D, E_{i}\right) \in \mathbb{Q}$ и $a\left(H, D, E_{1}\right) \leqslant-1$. Тогда бирациональный морфизм $f$ является композицией $k$ раздутий гладких точек. 
Предположим, что необходимое утверждение уже доказано во всех случаях, когда либо $a_{1} \leqslant 1$, либо $a_{2} \leqslant 1$. Следовательно, можно считать, что $a_{1}>1$ и $a_{2}>1$. Определим рациональные числа $a\left(H, E_{i}\right), m\left(H, M_{H}, E_{i}\right)$ и $m\left(H, \Delta_{j}, E_{i}\right)$ посредством соотношений $\sum_{i=1}^{k} a\left(H, E_{i}\right) E_{i} \sim_{\mathbb{Q}} K_{S}-f^{*}\left(K_{H}\right), \sum_{i=1}^{k} m\left(H, M_{H}, E_{i}\right) E_{i} \sim_{\mathbb{Q}}$ $f^{-1}\left(M_{H}\right)-f^{*}\left(M_{H}\right)$ и $\sum_{i=1}^{k} m\left(H, \Delta_{j}, E_{i}\right) E_{i} \sim_{\mathbb{Q}} f^{-1}\left(\Delta_{j}\right)-f^{*}\left(\Delta_{j}\right)$. Тогда

$$
\begin{aligned}
a\left(H, D, E_{i}\right)= & a\left(H, E_{i}\right)-m\left(H, M_{H}, E_{i}\right)+m\left(H, \Delta_{1}, E_{i}\right)\left(a_{1}-1\right) \\
& +m\left(H, \Delta_{2}, E_{i}\right)\left(a_{2}-1\right)
\end{aligned}
$$

и можно считать, что $m\left(H, \Delta_{1}, E_{1}\right) \geqslant m\left(H, \Delta_{2}, E_{1}\right)$. Таким образом, выполнено

$$
-1 \geqslant a\left(H, D, E_{1}\right) \geqslant a\left(H, E_{i}\right)-m\left(H, M_{H}, E_{i}\right)+m\left(H, \Delta_{2}, E_{i}\right)\left(a_{1}+a_{2}-2\right)
$$

и $O \in \mathbb{L} \mathbb{C} S\left(H,\left(2-a_{1}-a_{2}\right) \Delta_{2}+M_{H}\right)$. Значит, $\operatorname{mult}_{O}\left(M_{H}^{2}\right) \geqslant 4\left(a_{1}+a_{2}-1\right)$, так как по предположению утверждение теоремы вьполнено для $\left(H,\left(2-a_{1}-a_{2}\right) \Delta_{2}+M_{H}\right)$.

Можно считать, что $a_{1} \leqslant 1$. Пусть $h: T \rightarrow H$ - раздутие $O$, а $E-h$-исключительная кривая. Тогда $f=g \circ h$ для некоторого бирационального морфизма $g: S \rightarrow T$, состоящего из композиции $k-1$ раздутия гладких точек. В этом случае

$$
K_{T}+\left(1-a_{1}\right) \bar{\Delta}_{1}+\left(1-a_{2}\right) \bar{\Delta}_{2}+\left(1-a_{1}-a_{2}+m\right) E+M_{T} \sim_{\mathbb{Q}} h^{*}\left(K_{H}+D\right)
$$

где $\bar{\Delta}_{j}=h^{-1}\left(\Delta_{j}\right), m=\operatorname{mult}_{O}\left(M_{H}\right)$ и $M_{T}=h^{-1}\left(M_{H}\right)$.

Если $k=1$, то $S=T, E_{1}=E$ и $a\left(H, D, E_{1}\right)=a_{1}+a_{2}-m-1 \leqslant-1$. Таким образом,

$$
\operatorname{mult}_{O}\left(M_{H}^{2}\right) \geqslant m^{2} \geqslant\left(a_{1}+a_{2}\right)^{2} \geqslant 4 a_{1} a_{2}
$$

и необходимое утверждение доказано. Значит, можно считать, что выполнено неравенство $k>1$ и $P=g\left(E_{1}\right)$ - точка на кривой $E$.

По построению $P \in \mathbb{L} \mathbb{C S}\left(T,\left(1-a_{1}\right) \bar{\Delta}_{1}+\left(1-a_{2}\right) \bar{\Delta}_{2}+\left(1-a_{1}-a_{2}+m\right) E+M_{T}\right)$ и возможны три случая: $P \in E \cap \bar{\Delta}_{1}, P \in E \cap \bar{\Delta}_{2}$ и $P \notin \bar{\Delta}_{1} \cup \bar{\Delta}_{2}$. Можно считать, что утверждение теоремы выполнено для лог-пары $\left(T,\left(1-a_{1}\right) \bar{\Delta}_{1}+\left(1-a_{1}-\right.\right.$ $\left.\left.a_{2}+m\right) E+M_{T}\right)$ в случае, когда $P \in E \cap \bar{\Delta}_{1}$; для лог-пары $\left(T,\left(1-a_{2}\right) \bar{\Delta}_{2}+\right.$ $\left.\left(1-a_{1}-a_{2}+m\right) E+M_{T}\right)$ в случае, когда $P \in E \cap \bar{\Delta}_{2} ;$ для лог-пары $\left(T,\left(1-a_{1}-\right.\right.$ $\left.\left.a_{2}+m\right) E+M_{T}\right)$ в случае, когда $P \notin \bar{\Delta}_{1} \cup \bar{\Delta}_{2}$, поскольку все условия теоремы вьполнены в каждом из возможных случаев и бирациональный морфизм $g$ состоит из $k-1$ раздутия гладких точек. Более того, выполнено неравенство $\operatorname{mult}_{O}\left(M_{H}^{2}\right) \geqslant$ $m^{2}+\operatorname{mult}_{P}\left(M_{T}^{2}\right)$.

Рассмотрим случай $P \in E \cap \bar{\Delta}_{1}$. По индукции

$$
\operatorname{mult}_{O}\left(M_{H}^{2}\right) \geqslant m^{2}+4 a_{1}\left(a_{1}+a_{2}-m\right)=\left(2 a_{1}-m\right)^{2}+4 a_{1} a_{2} \geqslant 4 a_{1} 4 a_{2}
$$

Рассмотрим случай $P \in E \cap \bar{\Delta}_{2}$. При $a_{2} \leqslant 1$ или $a_{1}+a_{2}-m \leqslant 1$ необходимоенеравенство выводится как и в предыдушем случае. Таким образом, можно считать, что $a_{2}<1$ и $a_{1}+a_{2}-m<1$. Тогда

$$
\operatorname{mult}_{O}\left(M_{H}^{2}\right) \geqslant m^{2}+4\left(a_{1}+2 a_{2}-m-1\right)>4 a_{2} \geqslant 4 a_{1} 4 a_{2}
$$


Рассмотрим случай $P \notin \bar{\Delta}_{1} \cup \bar{\Delta}_{2}$. По индукции

$$
\operatorname{mult}_{O}\left(M_{H}^{2}\right) \geqslant m^{2}+4\left(a_{1}+a_{2}-m\right)>m^{2}+4 a_{1}\left(a_{1}+a_{2}-m\right) \geqslant 4 a_{1} 4 a_{2},
$$

что завершает доказательство требуемого утверждения.

Основные применения используют следующий частный случай теоремы 3.10 .

Лемма 3.11. Пусть $H$ - поверхность, $O$ - гладкая точка на $H, M_{H}-$ эффективная подвижная граница на $H$ и $O \in \mathbb{L} \mathbb{C S}\left(H, M_{H}\right)$. Tогда mult $O\left(M_{H}^{2}\right) \geqslant 4$ и выполнение равенства влечет mult $_{O}\left(M_{H}\right)=2$.

Следующий результат является следствием 7.3 работы [20].

Теорема 3.12. Пусть $X$ - трехмерное многообразие, $M_{X}$ - эффективная подвижная граница на многообразии $X$, а $O$ - гладкая точка на многообразии $X$ такая, что $O \in \mathbb{C S}\left(X, M_{X}\right)$. Тогда $\operatorname{mult}_{O}\left(M_{X}^{2}\right) \geqslant 4$ и выполнение равенства влечет mult $_{O}\left(M_{X}\right)=2$.

ДокАЗАТЕльСтво. Пусть $H$ - общее гиперплоское сечение многообразия $X$, проходящее через точку $O$. Тогда $O$ является центром лог-канонических особенностей лог-пары $\left(H,\left.M_{X}\right|_{H}\right)$ по теореме 3.9 . С другой стороны,

$$
\operatorname{mult}_{O}\left(M_{X}\right)=\operatorname{mult}_{O}\left(\left.M_{X}\right|_{H}\right), \quad \operatorname{mult}_{O}\left(M_{X}^{2}\right)=\operatorname{mult}_{O}\left(\left(\left.M_{X}\right|_{H}\right)^{2}\right)
$$

и необходимое утверждение следует из леммы 3.11 .

На самом деле утверждение теоремы 3.12 может быть естественно объяснено в намного более геометрическом контексте.

Лемма 3.13. Пусть $O$ - точка на гладком трехмерном многообразии $X$ такая, что $O \in \mathbb{C}\left(X, M_{X}\right)$, где $M_{X}$ - әффективная подвижсная граница на $X$, а особенности подвижной лог-пары $\left(X, M_{X}\right)$ каноничны. Тогда существует бирациональное отображение $f: V \rightarrow X$ такое, что $V$ имеет лишь терминальнье $\mathbb{Q}$-факториальнье особенности, бирачиональный морфизм $f$ стягивает ровно один исключительный дивизор $E$ в точку $O$ и $K_{V}+M_{V} \sim_{\mathbb{Q}} f^{*}\left(K_{X}+M_{X}\right)$, әде $M_{V}=f^{-1}\left(M_{X}\right)$.

ДоКАЗАТЕЛЬСТво. Количество различных дивизориальных нормирований $\nu$ поля рациональных функций многообразия $X$, чей центр на многообразии $X$ является точкой $O$ и чья дискрепанта $a\left(X, M_{X}, \nu\right)$ неположительна, конечно ввиду каноничности подвижной лог-пары $\left(X, M_{X}\right)$. Таким образом, можно рассмотреть бирациональный морфизм $g: W \rightarrow X$ такой, что трехмерное многообразие $W$ неособо, морфизм $g$ стягивает $k$ исключительных дивизоров

$$
K_{W}+M_{W} \sim_{\mathbb{Q}} g^{*}\left(K_{X}+M_{X}\right)+\sum_{i=1}^{k} a_{i} E_{i}
$$

лог-пара $\left(W, M_{W}\right)$ имеет канонические особенности, а множество $\mathbb{C} S\left(W, M_{W}\right)$ не содержит подмногообразий, содержашихся в $\bigcup_{i=1}^{k} E_{i}$, где $M_{W}=g^{-1}\left(M_{X}\right)$, 
$g\left(E_{i}\right)=O$ и числа $a_{i}$ рациональны. Применяя относительную лог-программу минимальных моделей к подвижной лог-паре $\left(W, M_{W}\right)$ над $X$, можно считать, что многообразие $W$ имеет лиш терминальные $\mathbb{Q}$-ракториальные особенности и $K_{W}+M_{W} \sim_{\mathbb{Q}} g^{*}\left(K_{X}+M_{X}\right)$, поскольку лог-пара $\left(X, M_{X}\right)$ имеет лишш канонические особенности. Применение относительной программы минимальных моделей к многообразию $W$ над $X$ доказывает требуемое утверждение.

Следуюший хорошо известный результат был первоначально сформулирован как гипотеза в работе [21], а затем доказан в работе [22].

Теорема 3.14. Пусть $X$ - гладкое трехмерное многообразие, $O$ - точ-

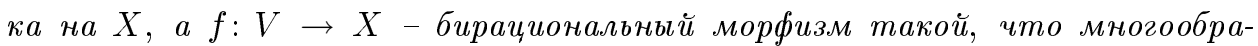
зие $V$ имеет лишь терминальные $\mathbb{Q}$-факториальные особенности, а морфизм $f$ стягивает ровно один исключительный дивизор $E$ и стягивает его в точку О. Тогда морфизм $f$ является взвешенным раздутием точки $О$ с весами $(1, K, N)$ в подходящих локальных координатах на многообразии $X$, где натуральные числа $K$ и $N$ взаимно простьи.

Более того, теорема 3.12 была доказана в [21] в предположении выполнения утверждения теоремы 3.14 следующим способом, которьй проясняет геометрическую природу неравенства в утверждении теоремы 3.12 .

УТВЕРЖДЕНИЕ 3.15. Пусть $X$ - гладкое трехмерное многообразие, $О$ точка на $X, M_{X}$ - әффективная подвижсная граница на $X$ такие, что $O \in \mathbb{C} S\left(X, M_{X}\right)$ и вылолнено соотношение $K_{V}+M_{V} \sim_{\mathbb{Q}} f^{*}\left(K_{X}+M_{X}\right)$, әде $f: V \rightarrow X$ - взвешенное раздутие точки $O$ с весами $(1, K, N)$ в соответствующих локальных координатах на гладком трехмерном многообразии $X$, где натуральные числа $L$ и взаимно просты $и M_{V}=f^{-1}\left(M_{X}\right)$. Тогда выполнено следующее:

$$
\operatorname{mult}_{O}\left(M_{X}^{2}\right) \geqslant \frac{(K+N)^{2}}{K N}=4+\frac{(K-N)^{2}}{K N} \geqslant 4
$$

а равенство $K=N$ возмохсно только в случае, когда морфизм $f$ является объкновенным раздутием точки $O$ и mult $_{O}\left(M_{X}\right)=2$.

ДокАЗАТЕЛЬСтво. Для исключительного дивизора $E$ морфизма $f$ и некоторого $m \in \mathbb{Q}_{>0} K_{V} \sim_{\mathbb{Q}} f^{*}\left(K_{X}\right)+(N+K) E$ и $M_{V} \sim_{\mathbb{Q}} f^{*}\left(M_{X}\right)+m E$. Таким образом, выполнено равенство $m=K+N$ и неравенство $\operatorname{mult}_{O}\left(M_{X}^{2}\right) \geqslant m^{2} E^{3}=$ $(K+N)^{2} /(K N)$.

\section{§4. Конструкция бирационального отображения $\rho_{i, k}$}

В обозначениях $\S 1$ пусть $g: W \rightarrow X$ - раздутие неособой кривой $Z_{1}^{0}, G$ - исключительньй дивизор морфизма $g$, а $\bar{Z}_{1}^{1}$ - собственный прообраз неособой рациональной кривой $Z_{1}^{1}$ на многообразии $W$. Тогда $K_{W} \cdot \bar{Z}_{1}^{1}=1$.

ЛЕмма 4.1. Справедливо следующее: $\mathscr{N}_{\bar{Z}_{1}^{1} / W} \cong \mathscr{O}_{\bar{Z}_{1}^{1}}(-1) \oplus \mathscr{O}_{\bar{Z}_{1}^{1}}(-2)$. 
ДокАЗАТЕЛЬСТВо. Пусть $\mathscr{N}_{\bar{Z}_{1}^{1} / W} \cong \mathscr{O}_{\bar{Z}_{1}^{1}}(m) \oplus \mathscr{O}_{\bar{Z}_{1}^{1}}(n)$ для $m \geqslant n$, а $H$ - достаточно общая поверхность в антиканонической линейной системе $\left|-K_{W}\right|$. Поверхность $H$ проходит через кривую $\bar{Z}_{1}^{1}$, поскольку $-K_{W} \cdot \bar{Z}_{1}^{1}=-1$. Отметим, что $\bar{Z}_{1}^{1}$ - единственная базисная кривая линейной системы $\left|-K_{W}\right|$. В частности, поверхность $H$ неособа вне кривой $\bar{Z}_{1}^{1}$ по теореме Бертини. С другой стороны, из обшности выбора поверхности $H$ и уравнения многообразия $V$ следует, что поверхность $f \circ g(H)$ неособа вне точки $O_{1}$, а в точке $O_{1}$ она имеет дювалевскую особенность типа $\mathbb{A}_{2}$. Из явного уравнения многообразия $X$ в окрестности кривых $Z_{1}^{0}$ и $Z_{1}^{1}$ следует неособость поверхности $g(H)$ вдоль кривых $Z_{1}^{0}$ и $Z_{1}^{1}$. В частности, ограничение бирационального морфизма $f$ на поверхность $g(H)$ стягивает две $(-2)$-кривые $Z_{1}^{0}$ и $Z_{1}^{1}$ в точку $O_{1}$. Неособость поверхности $g(H)$ влечет неособость $H$ вдоль $\bar{Z}_{1}^{1}$. Итак, $H$-гладкая поверхность $\mathrm{K} 3, m+n=-K_{W} \cdot \bar{Z}_{1}^{1}-2=-3$ и точная последовательность

$$
\left.0 \rightarrow \mathscr{N}_{\bar{Z}_{1}^{1} / H} \rightarrow \mathscr{N}_{\bar{Z}_{1}^{1} / W} \rightarrow \mathscr{N}_{H / W}\right|_{\bar{Z}_{1}^{1}} \rightarrow 0
$$

влечет $n \geqslant-2$. Таким образом, $n=-2$ и $m=-1$.

Пусть $h: U \rightarrow W$ - раздутие гладкой кривой $\bar{Z}_{1}^{1}, F$ - исключительный дивизор бирационального морфизма $h$, а $G_{U}-$ собственный прообраз дивизора $G$ на многообразии $U$. Тогда $F \cong \mathbb{F}_{1}, K_{U} \sim(g \circ h)^{*}\left(K_{X}\right)+F+G_{U}$, антиканоническая линейная система $\left|-K_{U}\right|$ свободна и морфизм $\varphi_{\left|-K_{U}\right|}: U \rightarrow \mathbb{P}^{2}$ является расслоением на эллиптические кривые с квазисечениями $F$ и $G_{U}$.

ЗАмечАние 4.2. Морфизм $\gamma \circ f \circ g \circ h: W \rightarrow \mathbb{P}^{3}$ отображает слои эллиптического расслоения $\varphi_{\left|-K_{U}\right|}$ на прямые в $\mathbb{P}^{3}$, проходящие через точку $O_{1}$, и расслоение $\varphi_{\left|-K_{U}\right|}$ индуцировано проекцией из точки $O_{1}$.

Пусть $C \subset U$ - исключительное сечение поверхности $F \cong \mathbb{F}_{1}$. Тогда $C$ стягивается в точку морфизмом $\varphi_{\left|-K_{U}\right|}: U \rightarrow \mathbb{P}^{2}$.

ЛЕмма 4.3. Справедливо следующее: $\mathscr{N}_{C / U} \cong \mathscr{O}_{C}(-1) \oplus \mathscr{O}_{C}(-1)$.

ДокАЗАТЕЛЬСТво. Пусть $\mathscr{N}_{C / U} \cong \mathscr{O}_{C}(m) \oplus \mathscr{O}_{C}(n)$ для $m \geqslant n$. Тогда выполнено равенство $m+n=-K_{U} \cdot C-2$, а точная последовательность

$$
\left.0 \rightarrow \mathscr{N}_{C / F} \rightarrow \mathscr{N}_{C / U} \rightarrow \mathscr{N}_{F / U}\right|_{C} \rightarrow 0
$$

влечет $n \geqslant-1$. Значит, $m=n=-1$.

Пусть $\widehat{p}: \widehat{U} \rightarrow U-$ раздутие гладкой кривой $C, R$ - исключительный дивизор морфизма $\widehat{p}$, а $\check{p}: \widehat{U} \rightarrow \breve{U}$ - такое сдутие поверхности $R \cong \mathbb{P}^{1} \times \mathbb{P}^{1}$ на кривую $\breve{C}$, что бирациональное отображение $\check{p} \circ \widehat{p}^{-1}$ не является бирегулярным. В этом случае бирациональное отображение $\check{p} \circ \widehat{p}^{-1}$ является флопом в кривой $C$, антиканоническая линейная система $\left|-K_{\check{U}}\right|$ свободна и $\varphi_{\left|-K_{\check{U}}\right|} \circ \check{p}=\varphi_{\left|-K_{U}\right|} \circ \widehat{p}$.

ЗАмечание 4.4 . Собственный прообраз $\breve{F} \subset \check{U}$ поверхности $F$ изоморфен $\mathbb{P}^{2}$ с нормальным расслоением $\mathscr{O}_{\mathbb{P}^{2}}(-2)$.

Поверхность $\check{F}$ может быть стянута $\breve{h}: \check{U} \rightarrow \breve{W}$ в особую точку $P \cong \frac{1}{2}(1,1,1)$. 
Лемма 4.5. Многообразие $\breve{W}$ проективно.

ДокАЗАтЕльСтво. Антифлип $\check{h} \circ \check{p} \circ \widehat{p}^{-1} \circ h^{-1}$ является лог-фолипом относительно лог-терминальной лог-пары $\left(W, \varepsilon\left|-K_{W}\right|\right)$ для достаточно малого рационального $\varepsilon>1$, откуда следует необходимое утверждение.

Лемма 4.6. Базисное множество линейной системь $\left|-K_{\check{W}}\right|$ состоит только из точки $P$, и выполнено равенство $-K_{\breve{W}}^{3}=\frac{1}{2}$.

ДокАЗАТЕЛЬСТво. Антиканоническая линейная система $\left|-K_{\breve{W}}\right|$ является собственным прообразом линейной системы $\left|-K_{\breve{U}}\right|$, откуда следует, что базисное множество линейной системы $\left|-K_{\breve{W}}\right|$ состоит из точки $P$. Равенства $K_{\breve{U}}^{3}=0$ и $\breve{F}^{3}=4$ и соотношение $K_{\breve{U}} \sim_{\mathbb{Q}} \breve{h}^{*}\left(K_{\breve{W}}\right)+\frac{1}{2} \check{F}$ влекут $-K_{\breve{W}}^{3}=\frac{1}{2}$.

Таким образом, дивизор $-K_{\breve{W}}$ является численно эффективным и объемным, линейная система $\left|-n K_{\breve{W}}\right|$ свободна для $n \gg 0$, морфизм $\varphi_{\left|-n K_{\breve{W}}\right|}: \check{W} \rightarrow V_{1,0}$ бирационален, а многообразие $V_{1,0}$ нормально. Построенное отображение

$$
\rho_{1,0}=\varphi_{\left|-n K_{\breve{W}}\right|} \circ \check{h} \circ \check{p} \circ \widehat{p}^{-1} \circ h^{-1} \circ g^{-1} \circ f^{-1}: V \rightarrow V_{1,0}
$$

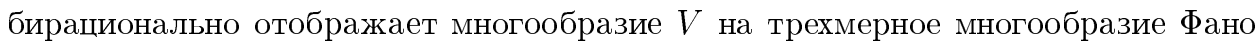
с каноническими особенностями $V_{1,0}$ такое, что выполнено равенство $-K_{V_{1,0}}^{3}=\frac{1}{2}$.

ЗАмечАниЕ 4.7. Особенности $V_{1,0}$ терминальны и не $\mathbb{Q}$-факториальны.

Конструкция отображений $\rho_{i, k}: V \rightarrow V_{i, k}$ идентична приведенной конструкции.

\section{§5. Доказательство теоремы 1.3}

В обозначениях и предположениях $\S 1$ предположим сушествование бирационального отображения $\beta: V \rightarrow Y$ и расслоения со связньми слоями $\pi: Y \rightarrow \mathbb{P}^{2}$, чей обший слой является гладкой эллиптической кривой. Допустим также, что не существует такого рационального отображения $\alpha: \mathbb{P}^{2} \rightarrow \mathbb{P}^{1}$, что $\alpha \circ \rho=\tau \circ f^{-1}$. В оставшейся части данного параграфа будет показано, что сделанные предположения приводят к противоречию. Положим $\rho=\pi \circ \beta$ и рассмотрим линейную систему $\left|\pi^{*}(D)\right|$ для очень обильного дивизора $D$ на $\mathbb{P}^{2}$. Пусть $\mathscr{D}_{V}=\beta^{-1}\left(\left|\pi^{*}(D)\right|\right)$. Тогда $\mathscr{D}_{V} \subset\left|-n K_{V}\right|$ для некоторого натурального числа $n$. Рассмотрим подвижную границу $\frac{1}{n} \mathscr{D}_{V}$.

Лемма 5.1. Справедливо следующее: $\overline{\mathbb{C S}}\left(V, \frac{1}{n} \mathscr{D}_{V}\right) \neq \varnothing$.

ДокАЗАтЕльство. Допустим, что лог-пара $\left(V, \frac{1}{n} \mathscr{D}_{V}\right)$ полутерминальна. Тогда для некоторого $\varepsilon \in \mathbb{Q}_{>1 / n}$ лог-пара $\left(V, \varepsilon \mathscr{D}_{V}\right)$ будет канонической моделью. Следовательно, вьполнено равенство $\varkappa\left(V, \varepsilon \mathscr{D}_{V}\right)=3$, но по построению линейной системы $\mathscr{D}_{V}$ для любого рационального $\varepsilon$ выполнено неравенство $\varkappa\left(V, \varepsilon \mathscr{D}_{V}\right) \leqslant 2$.

Таким образом, множество $\overline{\mathbb{C S}}\left(V, \frac{1}{n} \mathscr{D}_{V}\right)$ содержит точку или кривую на $V$.

ЗАмечАниЕ 5.2. По построению линейная система $\mathscr{D}_{V}$ не содержится в слоях никакого доминантного рационального отображения $\chi: V \rightarrow \mathbb{P}^{1}$. 
Лемма 5.3. Множество $\mathbb{C S}\left(V, \frac{1}{n} \mathscr{D}_{V}\right)$ не содержит гладких точек многообразия $V$.

ДокАЗАТЕЛЬСтво. Предположим, что $\mathbb{C} S\left(V, \frac{1}{n} \mathscr{D}_{V}\right)$ содержит точку $O$, которая не содержится в кривой $\widetilde{L}$. Рассмотрим общую поверхность $H_{O} \in\left|-K_{V}\right|$, проходящую через точку $O$. Тогда $2 n^{2}=H_{O} \cdot \mathscr{D}_{V}^{2} \geqslant n^{2} \operatorname{mult}_{O}\left(\mathscr{D}_{V}^{2}\right) \geqslant 4 n^{2}$ по теореме 3.12 .

Лемма 5.4. Пусть $\mathbb{C S}\left(V, \frac{1}{n} \mathscr{D}_{V}\right)$ содержит кривую $C \neq \widetilde{L}$. Тогда $-K_{V} \cdot C=1$.

ДокАЗАТЕЛЬСТво. Выполнение неравенства $\operatorname{mult}_{C}\left(\mathscr{D}_{V}\right) \geqslant n$ эквивалентно тому, что кривая $C$ содержится в множестве $\mathbb{C} S\left(V, \frac{1}{n} \mathscr{D}_{V}\right)$. Следовательно, для достаточно общего дивизора $H$ в свободной линейной системе $\left|-K_{V}\right|$ неравенства

$$
2 n^{2}=H \cdot \mathscr{D}_{V}^{2} \geqslant \operatorname{mult}_{C}\left(\mathscr{D}_{V}^{2}\right) H \cdot C \geqslant \operatorname{mult}_{C}^{2}\left(\mathscr{D}_{V}\right) H \cdot C \geqslant n^{2} H \cdot C
$$

влекут неравенство $-K_{V} \cdot C \leqslant 2$. Предположим, что $-K_{V} \cdot C=2$. Тогда выполнены равенства mult $C\left(\mathscr{D}_{V}^{2}\right)=\operatorname{mult}_{C}^{2}\left(\mathscr{D}_{V}\right)=n^{2}$ и носитель одномерного цикла $\mathscr{D}_{V}^{2}$ состоит в точности из кривой $C$. По построению $\varphi_{\mathscr{D}_{V}}(V)=\mathbb{P}^{2}$ и сушествует точка $P$ на многообразии $V$ такая, что $P \notin C$, а линейная подсистема $\mathscr{D}_{P} \subset \mathscr{D}_{V}$, состоящая из поверхностей в линейной системе $\mathscr{D}_{V}$, проходящих через точку $P$, не имеет неподвижных компонент. Следовательно, в теоретико-множественном смысле $P \in \mathscr{D}_{P}^{2} \subset \mathscr{D}_{V}^{2}=C$, что противоречит сделанному предположению $P \notin C$.

Лемма 5.5. Пусть $\mathbb{C S}\left(V, \frac{1}{n} \mathscr{D}_{V}\right)$ содержит кривую $C$. Тогда $C \cap \widetilde{L} \neq \varnothing$.

ДокАЗАТЕльСтво. Предположим, что $C \cap \widetilde{L}=\varnothing$. Тогда $\gamma(C) \subset \mathbb{P}^{3}-$ прямая, а ограничение $\left.\gamma\right|_{C}$ является изоморфизмом по лемме 5.4.

Допустим, что $\gamma(C) \not \subset S$. Рассмотрим линейную систему $\mathscr{H}_{C} \subset\left|-K_{V}\right|$, состояшую из поверхностей, проходящих через кривую $C$. Возьмем достаточно общую поверхность $H_{C}$ в линейной системе $\mathscr{H}_{C}$ и гладкую рациональную кривую $\widetilde{C}$ на многообразии $V$ такую, что $\widetilde{C} \neq C$ и $\gamma(\widetilde{C})=\gamma(C)$. По построению поверхность $H_{C}$ является гладкой поверхностью К3, которая содержит кривые $C$ и $\widetilde{C}$ и на ней вьполнены соотношения $C^{2}=\widetilde{C}^{2}=-2$ и $C \cdot \widetilde{C}=3$. Базисное множество линейной системы $\mathscr{H}_{C}$ состоит из $C \cup \widetilde{C}$, откуда

$$
\left.\mathscr{D}_{V}\right|_{H_{C}}=\operatorname{mult}_{C}\left(\mathscr{D}_{V}\right) C+\operatorname{mult}_{\widetilde{C}}\left(\mathscr{D}_{V}\right) \widetilde{C}+R_{H_{C}},
$$

где $R_{H_{C}}$ - подвижная граница на поверхности $H_{C}$. Таким образом, равенства

$$
n=\left.\mathscr{D}_{V}\right|_{H_{C}} \cdot \widetilde{C}=3 \operatorname{mult}_{C}\left(\mathscr{D}_{V}\right)-2 \operatorname{mult}_{\widetilde{C}}\left(\mathscr{D}_{V}\right)+R_{H_{C}} \cdot \widetilde{C}
$$

влекут mult $_{\widetilde{C}}\left(\mathscr{D}_{V}\right)=\operatorname{mult}_{C}\left(\mathscr{D}_{V}\right)=n$ и $R_{H_{C}}=\varnothing$. В частности, $\mathbb{C} S\left(V, \frac{1}{n} \mathscr{D}_{V}\right)$ содержит также и кривую $\widetilde{C}$. С другой стороны, из $R_{H_{C}}=\varnothing$ следует, что $\mathscr{D}_{V}$ содержится в слоях рационального отображения $\varphi_{\mathscr{H}_{C}}$, что невозможно.

Предположим теперь, что $\gamma(C) \subset S$. Раздуем $g: W \rightarrow V$ гладкую кривую $C$, обозначим через $G$ исключительньй дивизор раздутия $g$ и положим $\mathscr{D}_{W}=$ $g^{-1}\left(\mathscr{D}_{V}\right)$. Как легко видеть, антиканоническая линейная система $\left|-K_{W}\right|$ является 
пучком, а ее базисное множество состоит из гладкой рациональной кривой $\bar{C}$, которая является сечением линейчатой поверхности $\left.g\right|_{G}: G \rightarrow C$. Рассмотрим общую поверхность $H_{\bar{C}}$ в линейной системе $\left|-K_{W}\right|$. Поверхность $H_{\bar{C}}$ является неособой поверхностью К3 и

$$
\left.\mathscr{D}_{W}\right|_{H_{\bar{C}}}=\operatorname{mult}_{\bar{C}}\left(\mathscr{D}_{W}\right) \bar{C}+R_{H_{\bar{C}}}
$$

где $R_{H_{\bar{C}}}$ - подвижная граница на $H_{\bar{C}}$. На $H_{\bar{C}}$ вьполнены соотношения $\bar{C}^{2}=-2$ и

$$
\left.\mathscr{D}_{W}\right|_{H_{\bar{C}}} \sim_{\mathbb{Q}} n \bar{C}+\left.\left(n-\operatorname{mult}_{C}\left(\mathscr{D}_{V}\right)\right) G\right|_{H_{\bar{C}}},
$$

которые влекут mult ${ }_{\bar{C}}\left(\mathscr{D}_{W}\right)=\operatorname{mult}_{C}\left(\mathscr{D}_{V}\right)=n$ и $R_{H_{\bar{C}}}=\varnothing$. Теперь, как и в предыдущем случае, из пустоты $R_{H}$ следует, что линейная система $\mathscr{D}_{W}$ содержится в слоях отображения $\varphi_{\left|-K_{W}\right|}: W \rightarrow \mathbb{P}^{1}$, что невозможно по построению $\mathscr{D}_{V}$.

Лемма 5.6. Пусть $\widetilde{L} \notin \overline{\mathbb{C S}}\left(V, \frac{1}{n} \mathscr{D}_{V}\right)$. Тогда $\overline{\mathbb{C S}}\left(V, \frac{1}{n} \mathscr{D}_{V}\right)$ не содержит кривblx.

ДокАЗАТЕЛЬство. Кривая $\widetilde{L}$ не содержится в базисном множестве подвижной линейной системы $\mathscr{D}_{V}$, поскольку в противном случае множество $\overline{\mathbb{C}} \mathbb{S}\left(V, \frac{1}{n} \mathscr{D}_{V}\right)$ содержало бы кривую $\widetilde{L}$. В частности, собственный прообраз $\mathscr{D}_{X}$ линейной системы $\mathscr{D}_{V}$ на многообразии $X$ является подсистемой линейной системы $\left|-n K_{X}\right|$. Для доказательства необходимого утверждения достаточно показать, что множество $\mathbb{C} \mathbb{S}\left(X, \frac{1}{n} \mathscr{D}_{X}\right)$ не содержит кривых на многообразии $X$, которые не содержатся в исключительном дивизоре $E$. Предположим противное, а именно, что множество $\mathbb{C} \mathbb{S}\left(X, \frac{1}{n} \mathscr{D}_{X}\right)$ содержит кривую $C \subset X$, которая не лежит на поверхности $E$. Тогда из лемм 5.4 и 5.5 следует, что кривая $\gamma \circ f(C) \subset \mathbb{P}^{3}$ является прямой, ограничение $\left.\gamma \circ f\right|_{C}$ является изоморфизмом и $C \cap E \neq \varnothing$.

Предположим, что $\gamma \circ f(C) \not \subset S$. Рассмотрим линейную подсистему $\mathscr{H}_{C} \subset$ $\left|-K_{X}\right|$ поверхностей, проходящих через кривую $C$. Пусть $H_{C}$ - достаточно общая поверхность в линейной системе $\mathscr{H}_{C}, Z$ - слой $f$ над точкой $f(C) \cap \widetilde{L}$, а $\widetilde{C}$-гладкая кривая на многообразии $X$ такая, что $\widetilde{C} \neq C$ и $\gamma \circ f(\widetilde{C})=\gamma \circ f(C)$. Тогда $H_{C}-$ гладкая поверхность К3, содержашая кривые $C \cup \widetilde{C} \cup Z$, где $Z=Z_{i}^{0} \cup Z_{i}^{1}$ в случае $f(C) \cap \widetilde{L}=O_{i}$ и $Z$ - неприводимая кривая в противном случае. На поверхности $H_{C}$ вьполнены соотношения $Z^{2}=C^{2}=\widetilde{C}^{2}=-2, Z \cdot C=Z \cdot \widetilde{C}=1$ и $C \cdot \widetilde{C}=2$,

$$
\left.\mathscr{D}_{X}\right|_{H_{C}}=\operatorname{mult}_{C}\left(\mathscr{D}_{X}\right) C+\operatorname{mult}_{\widetilde{C}}\left(\mathscr{D}_{X}\right) \widetilde{C}+\Delta_{Z}+R_{H_{C}}
$$

поскольку базисное множество пучка $\mathscr{H}_{C}$ состоит из $Z \cup C \cup \widetilde{C}$, где $\Delta_{Z}-$ эффективный дивизор с носителем $Z$, а $R_{H_{C}}$ - подвижная граница на $H_{C}$. Более того, на поверхности $H_{C}$ выполнено соотношение

$$
R_{H_{C}} \sim_{\mathbb{Q}}\left(n-\operatorname{mult}_{C}\left(\mathscr{D}_{X}\right)\right) C+\left(n-\operatorname{mult}_{\widetilde{C}}\left(\mathscr{D}_{X}\right)\right) \widetilde{C}+n Z-\Delta_{Z}
$$

и форма пересечения кривых в $Z \cup \widetilde{C}$ отрицательно определена. Значит, $R_{H_{C}}=\varnothing$ и выполнены равенства mult $\widetilde{C}\left(\mathscr{D}_{X}\right)=\operatorname{mult}_{\widetilde{Z}}\left(\mathscr{D}_{X}\right)=n$. С другой стороны, из 
пустоты гранищы $R_{H_{C}}$ следует, что линейная система $\mathscr{D}_{X}$ содержится в слоях рационального отображения $\varphi \mathscr{H}_{C}$, что невозможно по построению линейной системы $\mathscr{D}_{V}$.

Предположим теперь, что $\gamma \circ f(C) \subset S$. Пусть $g: W \rightarrow X$ - раздутие гладкой кривой $C, G$ - исключительный дивизор раздутия $g$ и $\mathscr{D}_{W}=g^{-1}\left(\mathscr{D}_{X}\right)$. Тогда линейная система $\left|-K_{W}\right|$ является пучком, базисное множество пучка $\left|-K_{W}\right|$ состоит из кривых $\bar{Z}$ и $\bar{C}$, где $\bar{C}$ - сечение линейчатой поверхности $\left.g\right|_{G}: G \rightarrow C$, а $\bar{Z}$ - собственный прообраз на многообразии $W$ слоя бирационального морфизма $f$ над точкой $f(C) \cap \widetilde{L}$, состоящего из двух трансверсально пересекающихся в одной точке неособых рациональных кривых в случае, когда $f(C) \cap \widetilde{L}=O_{i}$, и из неособой рациональной кривой в противном случае. Рассмотрим общую поверхность $H_{\bar{C}} \in\left|-K_{W}\right|$. Она неособа и

$$
\left.\mathscr{D}_{W}\right|_{H_{\bar{C}}}=\operatorname{mult}_{\bar{C}}\left(\mathscr{D}_{W}\right) \bar{C}+\Delta_{\bar{Z}}+R_{H_{\bar{C}}}
$$

где $\Delta_{\bar{Z}}$ - эффективный дивизор с носителем $\bar{Z}$, а $R_{H_{\bar{C}}}$ - некоторая подвижная граница на поверхности $H_{\bar{C}}$. Более того, на поверхности $H_{\bar{C}}$ выполнено соотношение

$$
\left.\mathscr{D}_{W}\right|_{H_{\bar{C}}} \sim_{\mathbb{Q}} n \bar{Z}+n \bar{C}+\left.\left(n-\operatorname{mult}_{C}\left(\mathscr{D}_{X}\right)\right) G\right|_{H_{\bar{C}}}
$$

и во всех возможных случаях форма пересечения кривых в $\bar{C} \cup \bar{Z}$ отрицательно определена, откуда сразу следует, что подвижная граница $R_{H}$ пуста и вьполнены равенства mult $\bar{Z}_{\bar{Z}}\left(\mathscr{D}_{W}\right)=\operatorname{mult}_{\bar{C}}\left(\mathscr{D}_{W}\right)=\operatorname{mult}_{C}\left(\mathscr{D}_{X}\right)=n$. В частности, из пустоты подвижной границы $R_{H_{\bar{C}}}$ следует, что линейная система $\mathscr{D}_{W}$ содержится в слоях отображения $\varphi_{\left|-K_{W}\right|}: W \rightarrow \mathbb{P}^{1}$, что невозможно по построению линейной системы $\mathscr{D}_{V}$.

ЛЕмма 5.7. Предположим, ито $\overline{\mathbb{C S}}\left(V, \frac{1}{n} \mathscr{D}_{V}\right)$ не содержит кривой $\widetilde{L}$. Тогда множество $\overline{\mathbb{C S}}\left(V, \frac{1}{n} \mathscr{D}_{V}\right)$ не содержит точек на многообразии $V$, отличных от $O_{i}$.

ДоКАЗАТЕЛЬСТВо. Пусть $O$ - точка на многообразии $V$, отличная от точек $O_{i}$, которая содержится в $\overline{\mathbb{C S}}\left(V, \frac{1}{n} \mathscr{D}_{V}\right)$. Тогда $O \in \widetilde{L}$ по лемме 5.3 , но $\widetilde{L}$ не содержится в базисном множестве линейной системы $\mathscr{D}_{V}$, поскольку $\widetilde{L} \notin \overline{\mathbb{C S}}\left(V, \frac{1}{n} \mathscr{D}_{V}\right)$. В частности, собственный прообраз $\mathscr{D}_{X}$ линейной системы $\mathscr{D}_{V}$ на многообразии $X$ является линейной подсистемой линейной системы $\left|-n K_{X}\right|$, а $\left(X, \frac{1}{n} \mathscr{D}_{X}\right)$ является лог-поднятием лог-пары $\left(V, \frac{1}{n} \mathscr{D}_{V}\right)$. Значит, существует неприводимый слой $Z$ морфизма $\left.f\right|_{E}: E \rightarrow \widetilde{L}$ такой, что $\mathbb{C} \mathbb{S}\left(X, \frac{1}{n} \mathscr{D} X\right)$ содержит или сам слой, или точку на нем.

Предположим, что $\mathbb{C} S\left(X, \frac{1}{n} \mathscr{D}_{X}\right)$ содержит кривую $Z$. Пусть $g: W \rightarrow X-$ раздутие кривой $Z, \mathscr{D}_{W}-$ собственный прообраз $\mathscr{D}_{X}$ на $W$, а $G$ - исключительный дивизор бирационального морфизма $g$. Тогда

$$
\mathscr{D}_{W} \sim g^{*}\left(-n K_{X}\right)-\operatorname{mult}_{Z}\left(\mathscr{D}_{X}\right) G
$$

и mult $_{Z}\left(\mathscr{D}_{X}\right) \geqslant n$. С другой стороны, линейная система $\left|-K_{W}\right|$ свободна и задает расслоение $\varphi_{\left|-K_{W}\right|}: W \rightarrow \mathbb{P}^{2}$ на эллиптические кривые. Рассмотрим достаточно 
обший слой $C$ эллиптического расслоения $\varphi_{\left|-K_{W}\right|}$. Легко видеть, что выполнено равенство $\mathscr{D}_{W} \cdot C=2 n-2$ mult $_{Z}\left(\mathscr{D}_{X}\right)$. В частности, mult ${ }_{Z}\left(\mathscr{D}_{X}\right)=n$ и линейная система $\mathscr{D}_{W}$ содержится в слоях эллиптического расслоения $\varphi_{\left|-K_{W}\right|}$, что само по себе еще не является противоречием, однако показывает, что расслоения $\varphi_{\left|-K_{W}\right|}$ и $\pi$ эквивалентны. По построению сушествует проекция из точки $\alpha: \mathbb{P}^{2} \rightarrow \mathbb{P}^{1}$ такая, что выполнено соотношение $\alpha \circ \varphi_{\left|-K_{W}\right|} \circ(f \circ g)^{-1}=\tau \circ f^{-1}$, но это противоречит сделанному предположению.

Предположим теперь, что множество $\mathbb{C} S\left(X, \frac{1}{n} \mathscr{D}_{X}\right)$ содержит точку $P \in Z$. В этом случае положим $\mathscr{D}_{X}^{2}=\operatorname{mult}_{Z}\left(\mathscr{D}_{X}^{2}\right) Z+C_{X}$, где носитель одномерного эффективного цикла $C_{X}$ не содержит неприводимой кривой $Z$. Тогда

$$
\operatorname{mult}_{P}\left(\mathscr{D}_{X}^{2}\right)=\operatorname{mult}_{Z}\left(\mathscr{D}_{X}^{2}\right)+\operatorname{mult}_{P}\left(C_{X}\right) \geqslant 4 n^{2}
$$

по теореме 3.12. С другой стороны, $Z \cdot F=2$ и $\mathscr{D}_{X}^{2} \cdot F=2 n^{2}$, где $F-$ слой расслоения $\tau$ на поверхности дель Пецщо степени два. В частности, $\operatorname{mult}_{Z}\left(\mathscr{D}_{X}^{2}\right) \leqslant n^{2}$ и выполнено неравенство $\operatorname{mult}_{P}\left(C_{X}\right) \geqslant 3 n^{2}$. Возьмем обшую поверхность $H \in\left|-K_{X}\right|$, не содержашую кривой $Z$. Тогда поверхность $H$ не содержит компонент цикла $C_{X}$ и

$$
2 n^{2}=H \cdot C_{X} \geqslant \operatorname{mult}_{P}\left(C_{X}\right) \geqslant 3 n^{2},
$$

что доказывает требуемое утверждение.

Пусть $\mathscr{D}_{X}$ - собственный прообраз линейной системы $\mathscr{D}_{V}$ на гладком трехмерном многообразии $X$, а $F$ - слой расслоения $\tau: X \rightarrow \mathbb{P}^{1}$ на поверхности дель Пеццо. Тогда

$$
K_{X} \sim f^{*}\left(K_{V}\right), \quad F \sim-K_{X}-E, \quad \mathscr{D}_{X} \sim f^{*}\left(-n K_{V}\right)-m E,
$$

где $m=\operatorname{mult}_{\widetilde{L}}\left(\mathscr{D}_{V}\right)$.

ЛЕмма 5.8. Справедливо неравенство $m<n$.

ДоКАЗАТЕЛЬСТво. Из неравенства $m \geqslant n$ следует $\varphi_{\mathscr{D}_{X}}=\tau$, что невозможно.

Пусть $\mu=1 /(n-m)$ и $r=m /(n-m)$. Тогда $K_{X}+\mu \mathscr{D}_{X} \sim_{\mathbb{Q}} r F$.

ЗАмечАниЕ 5.9. Справедливо следуюшее:

$$
r>0 \Longleftrightarrow m>0 \Longleftrightarrow \widetilde{L} \in \overline{\mathbb{C S}}\left(V, \frac{1}{n} \mathscr{D}_{V}\right)
$$

Лемма 5.10. Справедливо соотношение $\mathbb{C} \mathbb{S}\left(X, \mu \mathscr{D}_{X}\right) \neq \varnothing$.

ДокАЗАТЕЛЬСтво. Предположим, что лог-пара $\left(X, \mu_{X}\right)$ имеет терминальные особенности. Тогда в случае $r>0$ лог-пара $\left(X, \varepsilon \mathscr{D}_{X}\right)$ является канонической моделью для некоторого $\varepsilon \in \mathbb{Q}_{>\mu}$ и, в частности, $\varkappa\left(X, \varepsilon \mathscr{D}_{X}\right)=3$, но $\varkappa\left(X, \varepsilon \mathscr{D}_{X}\right) \leqslant 2$ по построению линейной системы $\mathscr{D}_{V}$. В случае $r=0$ из сделанного предположения следует, что подвижная лог-пара $\left(V, \frac{1}{n} \mathscr{D}_{V}\right)$ полутерминальна, что невозможно по лемме 5.1 .

Следующий результат хорошо известен (см. [15]). 
ЛЕмма 5.11. Пусть $C$ - кривая в $\mathbb{C} S\left(X, \mu \mathscr{D}_{X}\right)$, которая содержится в слоях расслоения $\tau$ на поверхности дель Пещщо. Тогда лог-пара $\left(X, \mu_{X}\right)$ имеет лищь канонические особенности в общей точке кривой $C$.

ДокАЗАТЕЛьство. Пусть $F$ - слой расслоения $\tau$, содержащий $C$, а $L_{F}-$ достаточно общая кривая в слое $F$ такая, что образ $\gamma \circ f\left(L_{F}\right)$ является прямой в $\mathbb{P}^{3}$, содержашейся в плоскости $\gamma \circ f(F) \supset L$. Тогда

$$
2(n-m)=\mathscr{D}_{X} \cdot L_{F} \geqslant \operatorname{mult}_{C}\left(\mathscr{D}_{X}\right)\left(L_{F} \cdot C\right)_{F} \geqslant(n-m)\left(L_{F} \cdot C\right)_{F},
$$

откуда сразу следует, что либо образ $\gamma \circ f(C)$ кривой $C$ также является прямой и морфизм $\left.\gamma \circ f\right|_{C}: C \rightarrow \gamma \circ f(C)$ бирегулярен, либо $\operatorname{mult}_{C}\left(\mathscr{D}_{X}\right) \leqslant n-m$ и, в частности, лог-пара $\left(X, \mu \mathscr{D}_{X}\right)$ канонична в общей точке кривой $C$. Следовательно, можно считать, что кривая $\gamma \circ f(C)$ является прямой в $\mathbb{P}^{3}$ и $\left.\gamma \circ f\right|_{C}$ - изоморфизм.

Предположим, что слой $F$ особ в точке $O \in C$. Пусть $L_{O}-$ достаточно общая кривая на слое $F$ такая, что ее образ $\gamma \circ f\left(L_{O}\right)$ является прямой в $\mathbb{P}^{3}$, проходящей через точку $\gamma \circ f(O)$ и содержашейся в плоскости $\gamma \circ f(F)$, которая проходит через прямую $L$. Тогда кривая $L_{O}$ особа в точке $O$ и

$$
2(n-m)=\mathscr{D}_{X} \cdot L_{O} \geqslant \operatorname{mult}_{C}\left(\mathscr{D}_{X}\right) \operatorname{mult}_{O}\left(L_{O}\right) \geqslant 2(n-m),
$$

откуда следует, что mult $_{C}\left(\mathscr{D}_{X}\right)=n-m$ и лог-пара $\left(X, \mu \mathscr{D}_{X}\right)$ имеет лиш канонические особенности в общей точке кривой $C$.

Предположим, что поверхность $F$ неособа вдоль кривой $C$. Пусть $\widetilde{C}$ - гладкая рациональная кривая на поверхности $F$ такая, что $\widetilde{C} \neq C$ и $\gamma \circ f(\widetilde{C})=\gamma \circ f(C)$. Тогда

$$
\left.\mathscr{D}_{X}\right|_{F}=m_{C} C+m_{\widetilde{C}} \widetilde{C}+R_{F}
$$

для таких натуральных чисел $m_{C} \geqslant \operatorname{mult}_{C}\left(\mathscr{D}_{X}\right)$ и $m_{\widetilde{C}} \geqslant \operatorname{mult}_{\widetilde{C}}\left(\mathscr{D}_{X}\right)$, что $R_{F}-$ эффективный дивизор на $F$, чей носитель не содержит $C$ и $\widetilde{C}$. На $F$ выполнены соотношение

$$
\left.\mathscr{D}_{X}\right|_{F} \sim(n-m) C+(n-m) \widetilde{C}
$$

равенства $C^{2}=\widetilde{C}^{2}=-1$ и неравенства $R_{F} \cdot C \geqslant 0$ и $R_{F} \cdot \widetilde{C} \geqslant 0$, откуда следует, что выполнены неравенства $m_{C} \leqslant n-m$ и $m_{\widetilde{C}} \leqslant n-m$ и лог-пара $\left(X, \mu \mathscr{D}_{X}\right)$ имеет канонические особенности в общей точке кривой $C$.

Следующий результат хорошо известен и следует из результатов работ [4], [5].

Лемма 5.12. Существует такая композииия $\sigma$ инволющий Бертини общего слоя расслоения $\tau$, что лог-пара $\left(X, \mu_{\sigma} \sigma\left(\mathscr{D}_{X}\right)\right)$ имеет канонические особенности в общих точках кривых на $X$, где $\mu_{\sigma} \in \mathbb{Q}_{>0}$ задано соотношением $K_{X}+\mu_{\sigma} \sigma\left(\mathscr{D}_{X}\right) \sim_{\mathbb{Q}} r_{\sigma} F$ для некоторого неотрицательного рационального числа $r_{\sigma}$. 
ДокАЗАТЕЛЬСТво. Предположим, что $\operatorname{mult}_{C}\left(\mathscr{D}_{X}\right)>n-m$ для кривой $C$ на многообразии $X$. Кривая $C$ не содержится в слоях расслоения $\tau$ по лемме 5.11. Рассмотрим достаточно обший слой $F$ расслоения $\tau$. Тогда

$$
2(n-m)^{2}=\mathscr{D}_{X}^{2} \cdot F \geqslant \operatorname{mult}_{C}\left(\mathscr{D}_{X}^{2}\right) C \cdot F \geqslant \operatorname{mult}_{C}^{2}\left(\mathscr{D}_{X}\right) C \cdot F>(n-m)^{2} C \cdot F,
$$

откуда следует, что $C \cdot F=1$. Таким образом, можно временно считать, что $X$ - гладкая поверхность дель Пецшо степени два с группой Пикара $\mathbb{Z}$, которая определена над полем $\mathbb{F}=\mathbb{C}(x)$, кривую $C$ считать $\mathbb{F}$-точкой поверхности $X$, а $\mathscr{D}_{X}$ считать линейной системой кривых на поверхности $X$, не имеющей неподвижных кривых, причем выполнены соотношение $\mathscr{D}_{X} \subset\left|(m-n) K_{X}\right|$ и неравенство $\operatorname{mult}_{C}\left(\mathscr{D}_{X}\right)>n-m$.

Линейная система $\left|-K_{X}\right|$ не содержит особой в точке $C$ кривой $Z$, поскольку

$$
2(n-m)=Z \cdot \mathscr{D}_{X}>\operatorname{mult}_{C}(Z)(n-m),
$$

и, в частности, $\mathbb{F}$-точка $C$ не может содержаться в дивизоре ветвления двойного накрытия $\varphi_{\left|-K_{X}\right|}: X \rightarrow \mathbb{P}^{2}$. Пусть $g: W \rightarrow X$ - раздутие точки $C$, а $E$ - исключительная кривая раздутия $g$. Хорошо известно, что отображение $\varphi_{\left|-2 K_{W}\right|}$ является морфизмом и двойным накрытием. Рассмотрим инволюцию $\chi$ поверхности $W$, которая переставляет слои двойного накрытия $\varphi_{\left|-2 K_{W}\right|}$. Элементарные вычисления показывают, что

$$
\chi^{*}\left(g^{*}\left(-K_{X}\right)\right) \sim 3 g^{*}\left(-K_{X}\right)-4 E, \quad \chi^{*}(E) \sim 2 g^{*}\left(-K_{X}\right)-3 E,
$$

но в то же время хорошо известно, что бирегулярная инволюция $\chi$ индуцирует бирациональную и небирегулярную инволюцию $\psi_{C}$ поверхности $X$, которую принято называть инволюцией Бертини.

Рассмотрим $\mu_{\psi_{C}} \in \mathbb{Q}>0$ такое, что $K_{X}+\mu_{\psi_{C}} \psi_{C}\left(\mathscr{D}_{X}\right) \sim_{\mathbb{Q}} 0$. В этом случае

$$
\mu_{\psi_{C}}=\frac{\mu}{3-2 \operatorname{mult}_{C}\left(\mathscr{D}_{X}\right) \mu}
$$

откуда следует, что $\mu_{\psi_{C}}>\mu=1 /(n-m)$. Теперь можно повторить описанную вьше конструкцию инволюции Бертини, но уже для лог-пары $\left(X, \mu_{\psi_{C}} \psi_{C}\left(\mathscr{D}_{X}\right)\right)$ в случае, если ее особенности не являются каноническими. Таким образом, композиция $\sigma$ конечного числа инволюций Бертини поверхности дель Пецщо $X$ степени два задает подвижную лог-пару $\left(X, \mu_{\sigma} \sigma\left(\mathscr{D}_{X}\right)\right)$, где $K_{X}+\mu_{\sigma} \sigma\left(\mathscr{D}_{X}\right) \sim_{\mathbb{Q}} 0$ и $1 / \mu_{\sigma} \in \mathbb{N}$. Следовательно, рациональное число $\mu_{\sigma}$ можно считать минимальным среди возможных, откуда следует, что особенности лог-пары $\left(X, \mu_{\sigma} \sigma\left(\mathscr{D}_{X}\right)\right)$ канонические.

Теперь вновь рассмотрим $X$ как гладкое трехмерное многообразие. Предыдущая конструкция дает композицию $\sigma$ конечного числа инволюций Бертини общего слоя расслоения $\tau$ такую, что лог-пара $\left(X, \mu_{\sigma} \sigma\left(\mathscr{D}_{X}\right)\right)$ имеет лишш канонические особенности в общих точках кривых, не содержащихся в слоях расслоения $\tau$, где 
$\mu_{\sigma} \in \mathbb{Q}_{>0}$ такое, что $K_{X}+\mu_{\sigma} \sigma\left(\mathscr{D}_{X}\right) \sim_{\mathbb{Q}} r_{\sigma} F$ для некоторого рационального числа $r_{\sigma}$. Следовательно, можно применить леммы $5.1-5.6$ и $5.10,5.11$ к линейной системе без неподвижных компонент $f \circ \sigma\left(\mathscr{D}_{X}\right)$ вместо линейной системы $\mathscr{D}_{V}$, откуда будет следовать, что $r_{\sigma} \geqslant 0$ и особенности лог-пары $\left(X, \mu_{\sigma} \sigma\left(\mathscr{D}_{X}\right)\right)$ канонические в общих точках всех кривых на многообразии $X$.

Бирациональный автоморфизм $\sigma$ коммутирует с морфизмом $\tau$, следовательно, можно заменить бирациональное отображение $\beta$ на $\beta \circ \sigma^{-1}$ и считать, что особенности лог-пары $\left(X, \mu \mathscr{D}_{X}\right)$ каноничны в общих точках всех кривых на $X$.

Лемма 5.13. Справедливо неравенство $r>0$.

ДоказАтЕльство. Пусть $r=0$. Тогда $\widetilde{L} \notin \overline{\mathbb{C S}}\left(V, \frac{1}{n} \mathscr{D}_{V}\right)$ и $\overline{\mathbb{C S}}\left(V, \frac{1}{n} \mathscr{D}_{V}\right)$ содержит одну из точек $O_{i}$ по лемме 5.7. Можно считать, что $i=1$. Значит, $\mathbb{C S}\left(X, \frac{1}{n} \mathscr{D}_{X}\right)$ содержит либо точку $P \in Z_{1}$, либо компоненту кривой $Z_{1}=Z_{1}^{0} \cup Z_{1}^{1}$, где $Z_{1}$ - приводимый слой бирационального морфизма $f$ над точкой $O_{1}$. Рассмотрим две достаточно общие поверхности $D_{1}$ и $D_{2}$ в линейной системе $\mathscr{D}_{X}$. Тогда

$n^{2}\left(Z_{1}+2 C\right) \equiv n^{2} K_{X}^{2} \equiv D_{1} \cdot D_{2}=\operatorname{mult}_{Z_{1}^{0}}\left(\mathscr{D}_{X}^{2}\right) Z_{1}^{0}+\operatorname{mult}_{Z_{1}^{1}}\left(\mathscr{D}_{X}^{2}\right) Z_{1}^{1}+C_{X}+R_{X}$,

где $C$-кривая в слоях расслоения $\tau$ c $-K_{X} \cdot C=1, C_{X}$ - эффективный одномерный цикл на многообразии $X$, содержашийся в слоях расслоения $\tau$, а $R_{X}$ - эффективный одномерный цикл на $X$, чьи компоненты не содержатся в слоях расслоения $\tau$.

Допустим, что $\mathbb{C} S\left(X, \frac{1}{n} \mathscr{D}_{X}\right)$ содержит некоторую точку $P \in Z_{1}$. Тогда выполнено неравенство mult $_{P}\left(D_{1} \cdot D_{2}\right) \geqslant 4 n^{2}$ по теореме $3.12, Z_{1}^{0} \cdot F=Z_{1}^{1} \cdot F=1$ и

$$
2 n^{2}=D_{1} \cdot D_{2} \cdot F=\operatorname{mult}_{Z_{1}^{0}}\left(\mathscr{D}_{X}^{2}\right)+\operatorname{mult}_{Z_{1}^{1}}\left(\mathscr{D}_{X}^{2}\right)+R_{X} \cdot F,
$$

где $F$ - слой расслоения $\tau$. В частности, mult $Z_{1}^{0}\left(\mathscr{D}_{X}^{2}\right)+\operatorname{mult}_{Z_{1}^{1}}\left(\mathscr{D}_{X}^{2}\right) \leqslant 2 n^{2}$ и вьполнение равенства влечет $R_{X}=\varnothing$. Возьмем обшую поверхность $H_{Z_{1}}$ в $\left|-K_{X}\right|$, которая проходит через $Z_{1}$. В силу выбора можно считать, что $H_{Z_{1}}$ не содержит неприводимых компонент циклов $C_{X}$ и $R_{X}$. Таким образом,

$$
\begin{aligned}
H_{Z_{1}} \cdot\left(C_{X}+R_{X}\right) & \geqslant \operatorname{mult}_{P}\left(C_{X}\right)+\operatorname{mult}_{P}\left(R_{X}\right) \\
& \geqslant 4 n^{2}-\operatorname{mult}_{Z_{1}^{0}}\left(\mathscr{D}_{X}^{2}\right) \delta_{P}^{0}+\operatorname{mult}_{Z_{1}^{1}}\left(\mathscr{D}_{X}^{2}\right) \delta_{P}^{1}
\end{aligned}
$$

где $\delta_{P}^{i}=\operatorname{mult}_{P}\left(Z_{1}^{i}\right)$. Однако $H_{Z_{1}} \cdot\left(C_{X}+R_{X}\right)=2 n^{2}$. Следовательно, цикл $R_{X}$ пуст, выполнено равенство $\operatorname{mult}_{P}\left(C_{X}\right)=H_{Z_{1}} \cdot C_{X}=2 n^{2}$ и либо точка $P$ является точкой пересечения кривых $Z_{1}^{0}$ и $Z_{1}^{1}$ и выполнено равенство

$$
\text { mult }_{Z_{1}^{0}}\left(\mathscr{D}_{X}^{2}\right)+\operatorname{mult}_{Z_{1}^{1}}\left(\mathscr{D}_{X}^{2}\right)=2 n^{2}
$$

либо $P \in Z_{1}^{k}$, mult $Z_{1}^{k}\left(\mathscr{D}_{X}^{2}\right)=2 n^{2}$, mult $_{Z_{1}^{1-k}}\left(\mathscr{D}_{X}^{2}\right)=0$. Числа mult $Z_{1}^{0}\left(\mathscr{D}_{X}^{2}\right)$ и mult $_{Z_{1}^{1}}\left(\mathscr{D}_{X}^{2}\right)$ положительны, поскольку $D_{1} \cdot Z_{1}^{0}=D_{1} \cdot Z_{1}^{1}=0$, откуда следует, что $P=Z_{1}^{0} \cap Z_{1}^{1}$ и

$$
\text { mult }_{Z_{1}^{0}}\left(\mathscr{D}_{X}^{2}\right)+\operatorname{mult}_{Z_{1}^{1}}\left(\mathscr{D}_{X}^{2}\right)=2 n^{2}
$$


а из равенств mult $P\left(C_{X}\right)=H_{Z_{1}} \cdot C_{X}=2 n^{2}$ следует, что носитель цикла $C_{X}$ должен содержатся в слое $F_{P}$ расслоения $\tau$, который проходит через $P$. С другой стороны, по построению $\varphi_{\mathscr{D}_{X}}(X)=\mathbb{P}^{2}$, откуда следует сушествование точки $O \in X$ такой, что точка $O$ не содержится в объединении $F_{P} \cup E$, а линейная подсистема $\mathscr{D}_{O} \subset \mathscr{D}_{X}$, состоящая из поверхностей в линейной системе $\mathscr{D}_{X}$, проходящих через точку $O$, не имеет неподвижных компонент. Таким образом, в теоретикомножественном смысле

$$
O \in \mathscr{D}_{O}^{2} \subset \mathscr{D}_{V}^{2} \subset F_{P} \cup E
$$

что противоречит выбору точки $O$.

Предположим сначала, что реализуется простой случай и $\mathbb{C} S\left(X, \frac{1}{n} \mathscr{D} X\right)$ содержит обе кривые $Z_{1}^{0}$ и $Z_{1}^{1}$. Пусть $g: W \rightarrow X$ - раздутие кривой $Z_{1}^{0}, h: U \rightarrow W$ - раздутие собственного прообраза кривой $Z_{1}^{1}$, а $\mathscr{D}_{U}-$ собственный прообраз подвижной линейной системы $\mathscr{D}_{X}$ на многообразии $U$. Тогда линейная система $\left|-K_{U}\right|$ свободна, а индуцированный морфизм $\varphi_{\left|-K_{U}\right|}: U \rightarrow \mathbb{P}^{2}$ является расслоением на эллиптические кривые. Рассмотрим достаточно общий слой $C$ расслоения $\varphi_{\left|-K_{U}\right|}$. В этом случае

$$
\mathscr{D}_{U} \cdot C=2 n-\text { mult }_{Z_{1}^{0}}\left(\mathscr{D}_{X}\right)-\text { mult }_{Z_{1}^{1}}\left(\mathscr{D}_{X}\right)
$$

откуда сразу следует, что линейная система $\mathscr{D}_{U}$ содержится в слоях эллиптического расслоения $\varphi_{\left|-K_{U}\right|}$, которое эквивалентно исходному расслоению на эллиптические кривые $\pi$. Более того, из построения следует сушествование такого рационального отображения $\alpha: \mathbb{P}^{2} \rightarrow \mathbb{P}^{1}$, что $\alpha \circ \varphi_{\left|-K_{U}\right|} \circ(f \circ h \circ g)^{-1}=\tau \circ f^{-1}$, что противоречит одному из первоначальных предположений.

Теперь предположим, что множество $\mathbb{C} S\left(X, \frac{1}{n} \mathscr{D}_{X}\right)$ содержит только одну из неприводимых кривых $Z_{1}^{0}$ и $Z_{1}^{1}$. Без ограничения общности можно считать, что $\mathbb{C} \mathbb{S}\left(X, \frac{1}{n} \mathscr{D}_{X}\right)$ содержит кривую $Z_{1}^{0}$ и не содержит кривую $Z_{1}^{1}$. Пусть $g: W \rightarrow X-$ раздутие гладкой кривой $Z_{1}^{0}, G$ - исключительньй дивизор раздутия $g$, а $\mathscr{D}_{W}-$ собственный прообраз линейной системы $\mathscr{D}_{X}$ на многообразии $W$. Тогда лог-пара $\left(W, \frac{1}{n} \mathscr{D}_{W}\right)$ является лог-поднятием лог-пары $\left(X, \frac{1}{n} \mathscr{D}_{X}\right)$. В частности, особенности лог-пары $\left(W, \frac{1}{n} \mathscr{D}_{W}\right)$ также каноничны. Более того, в случае, когда особенности лог-пары $\left(W, \frac{1}{n} \mathscr{D}_{W}\right)$ не терминальны, сушествует кривая $C_{W} \subset W$ такая, что $C_{W} \in \mathbb{C} S\left(W, \frac{1}{n} \mathscr{D}_{W}\right)$ и $C_{W}$ не стягивается в точку морфизмом $g$, поскольку $\mathbb{C} \mathbb{S}\left(V, \frac{1}{n} \mathscr{D}_{V}\right)$ не содержит гладких точек многообразия $V$ по лемме 5.3 , множество $\mathbb{C} \mathbb{S}\left(X, \frac{1}{n} \mathscr{D}_{X}\right)$ не содержит точек в неприводимых слоях бирационального морфизма $f$ по лемме 5.7 и, как уже было показано ранее в доказательстве настоящего утверждения, $\mathbb{C} S\left(X, \frac{1}{n} \mathscr{D}_{X}\right)$ не содержит точек в приводимых слоях $Z_{i}$ морфизма $f$. Более того, $\mathbb{C} S\left(X, \frac{1}{n} \mathscr{D}_{X}\right)$ не может содержать кривых, отличных от кривых $Z_{i}^{k}$, по леммам 5.6, 5.7 и по предположению $C_{W}$ не может быть собственным прообразом кривой $Z_{1}^{1}$. Следовательно, либо кривая $C_{W}$ доминирует кривую $Z_{1}^{0}$, либо $C_{W}=Z_{j \neq 1}^{k}$. С другой стороны, $\left.D_{1}\right|_{H_{Z_{1}}} \cdot Z_{1}^{1}=0$, где $H_{Z_{1}}$ - обшая поверхность в $\left|-K_{X}\right|$, содержащая $Z_{1}$, откуда следует mult $Z_{1}^{1}\left(\mathscr{D}_{X}^{2}\right)>0$. Значит,

$$
2 n^{2}=D_{1} \cdot D_{2} \cdot F \geqslant \operatorname{mult}_{C_{W}}^{2}\left(\mathscr{D}_{W}\right)+\operatorname{mult}_{Z_{1}^{0}}^{2}\left(\mathscr{D}_{X}\right)+\operatorname{mult}_{Z_{1}^{1}}^{2}\left(\mathscr{D}_{X}\right)>2 n^{2}
$$


что невозможно. Таким образом, лог-пара $\left(W, \frac{1}{n} \mathscr{D}_{W}\right)$ имеет лишш терминальные особенности. В обозначениях $\S 4$ рассмотрим антифлип $\check{h} \circ \check{p} \circ \widehat{p}^{-1} \circ h^{-1}$ : $W \rightarrow \breve{W}$ в гладкой кривой $Z_{1}^{1}$ и обозначим через $\mathscr{D}_{\breve{W}}$ собственный прообраз $\mathscr{D}_{W}$ на $\check{W}$. В этом случае особенности лог-пары $\left(\breve{W}, \frac{1}{n} \mathscr{D}_{\breve{W}}\right)$ терминальны, поскольку $\left(K_{W}+\frac{1}{n} \mathscr{D}_{W}\right) \cdot Z_{1}^{1}=0$ и антифлип в $Z_{1}^{1}$ является лог-флопом относительно $\left(W, \frac{1}{n} \mathscr{D}_{W}\right)$. Следовательно, для достаточно малого $\varepsilon \in \mathbb{Q}_{>1 / n}$ особенности лог-пары $\left(\mathscr{W}, \varepsilon \mathscr{D}_{\breve{W}}\right)$ терминальны и

$$
K_{\breve{W}}+\varepsilon \mathscr{D}_{\breve{W}} \sim_{\mathbb{Q}}\left(\frac{1}{n}-\varepsilon\right) K_{\breve{W}},
$$

где дивизор $-K_{\breve{W}}$ численно эффективен и объемен по лемме 4.6. Более того, линейная система $\left|-n K_{\breve{W}}\right|$ задает бирациональный морфизм $\varphi_{\mid-n K_{\breve{W}}}: \check{W} \rightarrow V_{1,0}$ для некоторого $n \gg 0$, где $V_{1,0}$ - трехмерное особое многообразие Фано с $-K_{V_{1,0}}^{3}=\frac{1}{2}$, имеюшее только канонические особенности. Бирациональный морфизм $\varphi_{\mid-n K_{\breve{W}}} \mid$ стягивает только те кривые на многообразии $\breve{W}$, которые имеют тривиальное пересечение с дивизором $-K_{\check{W}}$. В частности, каждая кривая, стягиваемая морфизмом $\varphi_{\left|-n K_{\breve{W}}\right|}$, имеет тривиальное пересечение с дивизором $K_{\breve{W}}+\varepsilon \mathscr{D}_{\breve{W}}$. Значит, лог-пара $\left(\tilde{W}, \varepsilon \mathscr{D}_{\breve{W}}\right)$ является лог-поднятием лог-пары $\left(V_{1,0}, \varepsilon \mathscr{D}_{V_{1,0}}\right)$, где $\mathscr{D}_{V_{1,0}}-$ собственный прообраз линейной системы $\mathscr{D}_{\breve{W}}$ на $V_{1,0}$. Следовательно, особенности лог-пары $\left(V_{1,0}, \varepsilon \mathscr{D}_{V_{1,0}}\right)$ канонические и выполнено равенство $\varkappa\left(V_{1,0}, \varepsilon \mathscr{D}_{V_{1,0}}\right)=3$, но $\varkappa\left(V_{1,0}, \varepsilon \mathscr{D}_{V_{1,0}}\right) \leqslant 2$ по построению линейной системы $\mathscr{D}_{V}$.

Возьмем бирациональный морфизм $h: U \rightarrow X$ такой, что многообразие $U$ неособо, а отображение $\beta \circ f \circ h$ регулярно. Рассмотрим соотношение

$$
K_{U}+\mu \mathscr{D}_{U} \sim_{\mathbb{Q}} h^{*}(r F)+\sum_{i=1}^{k} a_{i} E_{i},
$$

где $\mathscr{D}_{U}-$ собственный прообраз линейной системы $\mathscr{D}_{X}$ на $U, E_{i}$ - исключительньй дивизор бирационального морфизма $h$ и $a_{i} \in \mathbb{Q}$. Пусть $\mathscr{J} \subset \mathbb{P}^{1}-$ конечное или пустое подмножество точек такое, что образ на многообразии $X$ хотя бы одного исключительного дивизора $E_{i}$, для которого вьполнено неравенство $a_{i}<0$, является точкой в слое расслоения $\tau$ над точкой множества $\mathscr{J}$. Для $\lambda \in \mathscr{J}$ положим

$$
h^{*}\left(F_{\lambda}\right) \sim h^{-1}\left(F_{\lambda}\right)+\sum_{j=1}^{k_{\lambda}} b_{j} E_{j},
$$

где $F_{\lambda}$ - слой расслоения $\tau$ над точкой $\lambda$ и $b_{i} \in \mathbb{N}$. Наконец, положим $\mathscr{I}=$ $\bigcup_{\lambda \in \mathscr{J}} \mathscr{I}_{\lambda}$, а для $\lambda \in \mathscr{J}$ определим множество $\mathscr{I}_{\lambda} \subset\{1, \ldots, k\}$ следующим образом: $i \in \mathscr{I}_{\lambda}$ в том и только том случае, если $h\left(E_{i}\right)$ является точкой в слое $F_{\lambda}$ и $a_{i}<0$.

Следующий результат - сушествование супермаксимальной особенности в $[15]$.

УТВЕРЖДЕНИЕ 5.14. Справедливо следующее:

$$
r+\sum_{\lambda \in \mathscr{J}} \min \left\{\frac{a_{i}}{b_{i}} \mid h\left(E_{i}\right) \in F_{\lambda} \quad u \quad a_{i}<0\right\} \leqslant 0 .
$$


ДокАЗАТЕльство. Предположим, что утверждение, которое требуется доказать, неверно. В этом случае существуют положительные рациональные числа $\varepsilon$ и $c_{\lambda}$ такие, что $r=\varepsilon+\sum_{\lambda \in \mathscr{J}} c_{\lambda}$ и $c_{\lambda}+\min \left\{a_{i} / b_{i} \mid h\left(E_{i}\right) \in F_{\lambda}\right.$ и $\left.a_{i}<0\right\}>0$. В частности,

$$
K_{U}+\mu \mathscr{D}_{U} \sim_{\mathbb{Q}} h^{*}(\varepsilon F)+\sum_{\lambda \in \mathscr{J}}\left(h^{*}\left(c_{\lambda} F_{\lambda}\right)+\sum_{i \in \mathscr{I}_{\lambda}} a_{i} E_{i}\right)+\sum_{i \notin \mathscr{I}} a_{i} E_{i},
$$

но для всех $\lambda \in \mathscr{J}$ дивизор $h^{*}\left(c_{\lambda} F_{\lambda}\right)+\sum_{i \in \mathscr{I}_{\lambda}} a_{i} E_{i}$ эффективен по выбору положительного рационального числа $c_{\lambda}$, а дивизор $\sum_{i \notin \mathscr{I}} a_{i} E_{i}$ эффективен, поскольку по предположению лог-пара $\left(X, \mu \mathscr{D}_{X}\right)$ имеет канонические особенности в общих точках кривых на многообразии $X$. Возьмем достаточно общую кривую $C$, лежашую в слоях морфизма $\rho \circ f \circ h$. Выполнено равенство $K_{U} \cdot C=0$, поскольку кривая $C$ эллиптическая, а из построения линейной системы $\mathscr{D}_{V}$ следует $\mathscr{D}_{U} \cdot C=0$. Таким образом,

$\left(K_{U}+\mu \mathscr{D}_{U}\right) \cdot C=h^{*}(\varepsilon F) \cdot C+\sum_{\lambda \in \mathscr{J}}\left(h^{*}\left(c_{\lambda} F_{\lambda}\right)+\sum_{i \in \mathscr{I}_{\lambda}} a_{i} E_{i}\right) \cdot C+\sum_{i \notin \mathscr{I}} a_{i} E_{i} \cdot C=0$

и, в частности, $h^{*}(\varepsilon F) \cdot C=0$ ввиду общности кривой $C$. Следовательно, сушествует рациональное отображение $\alpha: \mathbb{P}^{2} \rightarrow \mathbb{P}^{1}$ такое, что $\alpha \circ \rho=\tau \circ f^{-1}$, что противоречит сделанному предположению.

СлЕДСТВИЕ 5.15. Особенности лог-пары $\left(X, \mu \mathscr{D}_{X}\right)$ не являются каноническими.

ЗАмЕчАНИЕ 5.16. Геометрический смысл утверждения 5.14 незамысловат: для любых положительных рациональных чисел $\left\{c_{\lambda}\right\}_{\lambda \in \mathscr{J}}$ с условием $r=\sum_{\lambda \in \mathscr{J}} c_{\lambda}$ множество центров канонических особенностей $\mathbb{C} S\left(X, \mu \mathscr{D}_{X}-\sum_{\lambda \in \mathscr{J}} c_{\lambda} F_{\lambda}\right)$ непусTO.

Рассмотрим неприводимую гладкую кривую $Z$, которая является общим слоем морфизма $\left.f\right|_{E}: E \rightarrow \widetilde{L}$, и неприводимую гладкую кривую $C$, лежащую в общем слое расслоения $\tau$ на поверхности дель Пещо такую, что $-K_{X} \cdot C=1$.

ЗАмечание 5.17. Справедливо следующее: $\overline{\mathbb{N E}}(X)=\mathbb{R}_{\geqslant 0} Z \oplus \mathbb{R}_{\geqslant 0} C, F \cdot Z=$ $E \cdot C=2, K_{X}^{2} \equiv Z+2 C$.

Отметим, что $\mathbb{Q}$-рациональная эквивалентность $\mu_{X} \sim_{\mathbb{Q}}-K_{X}+r F$ влечет численную эквивалентность одномерных циклов $\mu^{2} \mathscr{D}_{X}^{2} \equiv Z+(2+4 r) C$. Для двух достаточно общих дивизоров $D_{1}$ и $D_{2}$ в линейной системе $\mathscr{D}_{X}$ рассмотрим эффективный цикл

$$
T_{0}=\mu^{2} D_{1} \cdot D_{2}=Z_{X}+\sum_{\lambda \in \mathbb{P}^{1}} C_{\lambda}
$$

где все компоненты эффективного одномерного цикла $Z_{X}$ не содержатся в слоях расслоения $\tau$ на поверхности дель Пещц, а каждая компонента эффективного одномерного цикла $C_{\lambda}$ лежит в слое $F_{\lambda}$ расслоения $\tau$ над точкой $\lambda \in \mathbb{P}^{1}$. 
ЗАмечаниЕ 5.18. Имеет место следующее:

$$
-K_{X} \cdot \sum_{\lambda \in \mathbb{P}^{1}} C_{\lambda}=2+4 r \leqslant 2+4 \sum_{\lambda \in \mathscr{J}} \max \left\{\frac{-a_{i}}{b_{i}} \mid h\left(E_{i}\right) \in F_{\lambda} \text { и } a_{i}<0\right\} .
$$

СлеДСТвИЕ 5.19. Существуют точка $\omega \in \mathbb{P}^{1}$ и h-исключительный дивизор $E_{t}$ такие, что $h\left(E_{t}\right)$ является точкой $O$ в слое $F_{\omega}$ расслоения $\tau$ над точкой $\omega$, выполнены неравенства $-K_{X} \cdot C_{\omega} \leqslant 2-4 a_{t} / b_{t}$ и $a_{t}<0$, где $a_{t} / b_{t}=\min \left\{a_{i} / b_{i} \mid h\left(E_{i}\right) \in F_{\omega} \quad u \quad a_{i}<0\right\}$.

ЗАмечание 5.20. Справедливо следуюшее: $-K_{X} \cdot C_{\omega} \leqslant 2 / p-4 a_{t} / b_{t}$, где $p-$ мощность множества $\mathscr{J}$.

ЗАмечАние 5.21. Лог-пара $\left(X, \mu \mathscr{D}_{X}+\left(a_{t} / b_{t}\right) F_{\lambda}\right)$ канонична в $O$, а в соотношении

$$
K_{U}+\mu \mathscr{D}_{U}+\frac{a_{t}}{b_{t}} h^{-1}\left(F_{\omega}\right) \sim_{\mathbb{Q}} h^{*}\left(\left(r+\frac{a_{t}}{b_{t}}\right) F\right)+\sum_{i=1}^{k} a\left(X, \mu \mathscr{D}_{X}+\frac{a_{t}}{b_{t}} F_{\lambda}, E_{i}\right) E_{i}
$$

лог-дискрепанта $a\left(X, \mu \mathscr{D}_{X}+\left(a_{t} / b_{t}\right) F_{\lambda}, E_{t}\right)$ равна нулю, откуда следует, что точка $O$ содержится в множестве $\mathbb{C}\left(X, \mu \mathscr{D}_{X}+\left(a_{t} / b_{t}\right) F_{\lambda}\right)$ и сушествует только конечное число дивизориальных дискретных нормирований с центром в точке $O$ на $X$, у которых соответствующие дискрепанты обращаются в нуль, причем все такие нормирования индуцированы $h$-исключительными дивизорами $E_{j}$, для которых $a_{j} / b_{j}=a_{t} / b_{t}$, и вместо дивизора $E_{t}$ в следствии 5.19 можно взять любой из них.

Итак, имеются точка $\omega$ на $\mathbb{P}^{1}$, слой $F_{\omega}$ расслоения $\tau: X \rightarrow \mathbb{P}^{1}$ на поверхности дель Пеццо степени два над точкой $\omega$, гладкое многообразие $U$ и бирациональный морфизм $h: U \rightarrow X, h$-исключительньй дивизор $E_{t}$, чей образ $h\left(E_{t}\right)$ на $X$ является точкой $O$ на слое $F_{\omega}$, выполнены неравенства $-K_{X} \cdot C_{\omega} \leqslant 2-4 a_{t} / b_{t}$ и $a_{t}<0$, где одномерный эффективный цикл $C_{\omega}$ является компонентой цикла $T_{0}=\mu^{2} D_{1} \cdot D_{2}$, лежащей в слое $F_{\omega}$, а $D_{1}$ и $D_{2}-$ достаточно общие дивизоры в $\mathscr{D}_{X}$, рациональное число $a_{t}$ определено посредством $\mathbb{Q}$-рациональной эквивалентности

$$
K_{U}+\mu \mathscr{D}_{U} \sim_{\mathbb{Q}} h^{*}(r F)+\sum_{i=1}^{k} a_{i} E_{i},
$$

а натуральное число $b_{t}$ определено посредством рациональной эквивалентности

$$
h^{*}\left(F_{\omega}\right) \sim h^{-1}\left(F_{\omega}\right)+\sum_{i=1}^{k} b_{i} E_{i},
$$

где $\mathscr{D}_{U}=h^{-1}\left(\mathscr{D}_{X}\right)$, дивизор $E_{i}$ является $h$-исключительным, $a_{i} \in \mathbb{Q}$ и $b_{i} \in \mathbb{N}$.

Лемма 5.22. Слой $F_{\omega}$ неособ в точке $O$.

ДокАЗАТЕЛЬСтво. Предположим, что $F_{\omega}$ имеет особенность в $O$. Пусть $L_{O}-$ достаточно обшая кривая на поверхности $F_{\omega}$, чей образ $\gamma \circ f\left(L_{O}\right)$ является прямой в $\mathbb{P}^{3}$, проходящей через точку $\gamma \circ f(O)$. Тогда кривая $L_{O}$ имеет особенность в точке $O$ и

$$
2(n-m)=\mathscr{D}_{X} \cdot L_{O} \geqslant \operatorname{mult}_{O}\left(\mathscr{D}_{X}\right) \operatorname{mult}_{O}\left(L_{O}\right) \geqslant 2 \operatorname{mult}_{O}\left(\mathscr{D}_{X}\right),
$$

но неравенство $a_{t}<0$ влечет $\operatorname{mult}_{O}\left(\mathscr{D}_{X}\right)>\frac{1}{\mu}=n-m$. 
ЛЕмма 5.23. Справедливо следующее: $\operatorname{mult}_{O}\left(\mathscr{D}_{X}\right) \leqslant \frac{2}{\mu}=2(n-m)$.

ДоКАЗАТЕЛЬСтво. Пусть $L_{O}$ - достаточно общая кривая на поверхности $F_{\omega}$, содержащая точку $O$, чей образ $\gamma \circ f\left(L_{O}\right)$ является прямой в $\mathbb{P}^{3}$. Тогда

$$
2(n-m)=\mathscr{D}_{X} \cdot L_{O} \geqslant \operatorname{mult}_{O}\left(\mathscr{D}_{X}\right) \operatorname{mult}_{O}\left(L_{O}\right) \geqslant \operatorname{mult}_{O}\left(\mathscr{D}_{X}\right),
$$

откуда следует требуемое утверждение.

Числа $a_{t}$ и $b_{t}$ зависят только от дивизориального дискретного нормирования, которое индуцировано дивизором $E_{t}$, они являются лог-дискрепантой лог-пары $\left(X, \mu \mathscr{D}_{X}\right)$ и кратностью слоя $F_{\omega}$ соответственно. В частности, $a_{t}$ и $b_{t}$ не зависят от выбора бирационального морфизма $h$. Следовательно, можно считать, что $h$ является композицией $\psi_{1,0} \circ \cdots \circ \psi_{N, N-1}$ морфизмов $\psi_{i, i-1}: X_{i} \rightarrow X_{i-1}$, $i=1, \ldots, N, X=X_{0}$, таких, что выполнены следующие естественные требования: бирациональный морфизм $\psi_{1,0}$ является раздутием точки $P_{0}=O$; бирациональный морфизм $\psi_{i, i-1}$ является раздутием точки $P_{i-1} \in G_{i-1}, i=2, \ldots, K$, где $G_{i}=\psi_{i, i-1}^{-1}\left(P_{i-1}\right)$; бирациональный морфизм $\psi_{K+1, K}$ является раздутием кривой $P_{K} \subset G_{K}$; морфизм $\psi_{i, i-1}$ является раздутием кривой $P_{i-1} \subset G_{i-1}$, $i=K+2, \ldots, N$, которая не содержится в слоях морфизма $\left.\psi_{i-1, i-2}\right|_{G_{i-1}} ;$ дивизориальное дискретное нормирование, ассоциированное с дивизором $G_{N}$, совпадает с дивизориальным дискретным нормированием, которое ассоциировано с дивизором $E_{t}$.

Обозначим $\psi_{j, i}=\psi_{i+1, i} \circ \cdots \circ \psi_{j, j-1}: X_{j} \rightarrow X_{i}, j>i, \psi_{j, j}=\mathrm{id}_{X_{j}}$, а затем положим $G_{i}^{j}=\psi_{j, i}^{-1}\left(G_{i}\right) \subset X_{j}, j \geqslant i$, и $\mathscr{D}_{X_{i}}=\psi_{i, 0}^{-1}\left(\mathscr{D}_{X}\right), F_{\omega}^{i}=\psi_{i, 0}^{-1}\left(F_{\omega}\right) \subset X_{i}$, $i=0, \ldots, N$. В принятых обозначениях вьполнены соотношения

$$
\begin{gathered}
K_{X_{N}}+\mu \mathscr{D}_{X_{N}} \sim_{\mathbb{Q}} \psi_{N, 0}^{*}\left(r F_{\omega}\right)+\sum_{i=1}^{N} a\left(X, \mu \mathscr{D}_{X}, G_{i}\right) G_{i}^{N} \\
\psi_{N, 0}^{*}\left(F_{\omega}\right) \sim F_{\omega}^{N}+\sum_{i=1}^{N} b\left(X, F_{\omega}, G_{i}\right) G_{i}^{N}
\end{gathered}
$$

где $a\left(X, \mu \mathscr{D}_{X}, G_{i}\right) \in \mathbb{Q}$ и $b\left(X, F_{\omega}, G_{i}\right) \in \mathbb{N}$, а по построению $a\left(X, \mu \mathscr{D}_{X}, G_{N}\right)=a_{t}$, $b\left(X, F_{\omega}, G_{N}\right)=b_{t}$,

$$
-K_{X} \cdot C_{\omega} \leqslant 2-\frac{4 a\left(X, \mu \mathscr{D}_{X}, G_{N}\right)}{b\left(X, F_{\omega}, G_{N}\right)}
$$

и $a\left(X, \mu \mathscr{D}_{X}, G_{N}\right)<0$. Более того, как было отмечено в замечании 5.21 , можно считать, что $a\left(X, \mu \mathscr{D}_{X}, G_{N}\right) / b\left(X, F_{\omega}, G_{N}\right)<a\left(X, \mu \mathscr{D}_{X}, G_{i}\right) / b\left(X, F_{\omega}, G_{i}\right), i<N$.

ЗАмечАниЕ 5.24. Априори следует считать многообразие $X_{i}$ либо квазипроективным, либо особым при $i>K$, что нисколько не влияет на ход дальнейших рассуждений, однако следует отметить, что из неравенства $a\left(X, \mu \mathscr{D}_{X}, G_{N}\right)<0$, замечания 5.21 и леммы 5.23 нетрудно вывести, что $P_{K}$ является прямой в $G_{K} \cong \mathbb{P}^{2}$, а $P_{i}$ является сечением линейчатой поверхности $\left.\psi_{i-1, i-2}\right|_{G_{i-1}}: G_{i-1} \rightarrow P_{i-1} \cong \mathbb{P}^{1}$ при $i>K$. 
Основной объект оставшейся части параграфа - эффективный одномерный цикл

$$
T_{0}=\mu^{2} D_{1} \cdot D_{2}=Z_{X}+C_{\omega}+\sum_{\omega \neq \lambda \in \mathbb{P}^{1}} C_{\lambda}
$$

где компоненты цикла $Z_{X}$ не лежат в слоях расслоения $\tau$, а каждая компонента цикла $C_{\lambda}$ содержится в слое $F_{\lambda}$ расслоения $\tau$ над точкой $\lambda \neq \omega$ и $C_{\omega} \subset F_{\omega}$.

Из неравенства $a\left(X, \mu \mathscr{D}_{X}, G_{K}\right)<0$ следует $\operatorname{mult}_{O}\left(T_{0}\right)>4$ по теореме 3.12 . $\mathrm{C}$ другой стороны, выполнено неравенство $\operatorname{mult}_{O}\left(Z_{X}\right) \leqslant F_{\omega} \cdot Z_{X}=2$, откуда mult $_{O}\left(C_{\omega}\right)>2$, что само по себе является достаточно сильным утверждением, но недостаточным для получения желаемого противоречия.

ЗАмЕЧАнИЕ 5.25. Справедливо

$$
2<\operatorname{mult}_{O}\left(C_{\omega}\right) \leqslant-K_{X} \cdot C_{\omega} \leqslant 2-\frac{4 a\left(X, \mu \mathscr{D}_{X}, G_{N}\right)}{b\left(X, F_{\omega}, G_{N}\right)}
$$

Следующая конструкция хорошо известна (см. [15] и [20]). Сопоставим каждому дивизору $G_{i}$ вершину ориентированного графа $\Gamma$. Пусть стрелка $G_{j} \rightarrow G_{i}$ обозначает тот факт, что вершины $G_{j}$ и $G_{i}$ графа $\Gamma$ соединены направленньм ребром, идушим от вершины $G_{j}$ к вершине $G_{i}$. Положим

$$
G_{j} \rightarrow G_{i} \Longleftrightarrow j>i \text { и } P_{j-1} \subset G_{i}^{j-1} \subset X_{j-1}
$$

так что, в частности, всегда $G_{i} \rightarrow G_{i-1}$ для $i=1, \ldots, N$.

ЗАмЕчАниЕ 5.26. В случае, когда дискретное нормирование дивизора $G_{N}$ совпадает с дискретным нормированием исключительного дивизора взвешенного раздутия точки $O$ с весами $(1, K, N)$ для взаимно простых натуральных чисел $K$ и $N$ в подходящих локальных координатах на многообразии $X$, из работы [22] следует, что ориентированньй графф Г является цепью, что просто означает $G_{j} \rightarrow G_{i} \Longleftrightarrow$ $j=i+1$.

Пусть $P(i)$ - число путей в $\Gamma$, идуших из $G_{N}$ в $G_{i}, i \neq N$, и $P(N)=1$. Тогда

$$
a\left(X, \mu \mathscr{D}_{X}, G_{N}\right)=\sum_{i=1}^{K} P(i)\left(2-\nu_{i}\right)+\sum_{i=K+1}^{N} P(i)\left(1-\nu_{i}\right),
$$

где $\nu_{i}=\operatorname{mult}_{P_{i-1}}\left(\mu_{X}^{i-1}\right)$. Положим

$$
T_{0}^{j}=\psi_{j, 0}^{-1}\left(T_{0}\right), \quad Z_{X}^{j}=\psi_{j, 0}^{-1}\left(Z_{X}\right), \quad C_{\omega}^{j}=\psi_{j, 0}^{-1}\left(C_{\omega}\right)
$$

и

$$
m_{T_{0}}^{i}=\operatorname{mult}_{P_{i-1}}\left(T_{0}^{i-1}\right), \quad m_{Z_{X}}^{i}=\operatorname{mult}_{P_{i-1}}\left(Z_{X}^{i-1}\right), \quad m_{C_{\omega}}^{i}=\operatorname{mult}_{P_{i-1}}\left(C_{\omega}^{i-1}\right),
$$

где $i=1, \ldots, K$. Пусть $D_{1}^{i}=\psi_{i, 0}^{-1}\left(D_{1}\right), D_{2}^{i}=\psi_{i, 0}^{-1}\left(D_{2}\right)$ и $T_{i} \subset G_{i} \cong \mathbb{P}^{2}$-эффективный одномерный цикл на многообразии $X_{i}, i=1, \ldots, N$, заданный соотношением

$$
\mu^{2} \mathscr{D}_{X_{i}}^{2} \equiv \mu^{2} D_{1}^{i} \cdot D_{2}^{i}=\psi_{i, i-1}^{-1}\left(\mu^{2} D_{1}^{i-1} \cdot D_{2}^{i-1}\right)+T_{i},
$$


положим $T_{i}^{j}=\psi_{j, i}^{-1}\left(T_{i}\right)$ при $j \geqslant i$. Тогда для $i=1, \ldots, K$ вьполнено соотношение

$$
\mu^{2} D_{1}^{i} \cdot D_{2}^{i}=T_{0}^{i}+T_{1}^{i}+\cdots+T_{i-1}^{i}+T_{i}
$$

Пусть $m_{i, j}=\operatorname{mult}_{P_{j-1}}\left(T_{i}^{j-1}\right)$ и $d_{i}-$ степень цикла $T_{i}$ в $\mathbb{P}^{2} \cong G_{i}, j>i=$ $1, \ldots, K$.

ЛЕмма 5.27. Для $i=1, \ldots, K \quad \sum_{j=0}^{i-1} m_{j, i}=\nu_{i}^{2}+d_{i}$.

ДокАЗАтельство. Ограничим все на общее гиперплоское сечение трехмерного многообразия $X_{i-1}$, проходящего через точку $P_{i-1}$. Тогда необходимое утверждение сводится к известному результату о кратности пересечения на гладкой поверхности.

ЛЕмма 5.28. Справедливо неравенство $d_{K} \geqslant \sum_{i=K+1}^{N} \nu_{i}^{2} \operatorname{deg}\left(\left.\psi_{i-1, L}\right|_{P_{i-1}}\right)$.

ДокАЗАТЕЛьство. При $i>K$ рассмотрим $T_{i}$ как подсхему в $G_{i}$. Пусть $d_{i}-$ пересечение $T_{i}$ в $G_{i}$ с общим слоем морфизма $\left.\psi_{i, i-1}\right|_{G_{i}}$. Тогда $d_{i} \geqslant \operatorname{mult}_{P_{i}}\left(\mathscr{D}_{X_{i}}^{2}\right) \times$ $\operatorname{deg}\left(\left.\psi_{i, i-1}\right|_{P_{i}}\right)$ для $i>K$ и $\operatorname{mult}_{P_{i}}\left(\mathscr{D}_{X_{i}}^{2}\right)=\nu_{i+1}^{2}+d_{i+1}$ для $i \geqslant K$. Значит, выполнены неравенства

$$
\begin{aligned}
d_{K} \geqslant \operatorname{mult}_{P_{K}}\left(\mathscr{D}_{X_{L}}^{2}\right) & \geqslant \sum_{i=K+1}^{N} \nu_{i}^{2} \prod_{j=K}^{i-1} \operatorname{deg}\left(\left.\psi_{j+1, j}\right|_{P_{j+1}}\right) \\
& =\sum_{i=K+1}^{N} \nu_{i}^{2} \operatorname{deg}\left(\left.\psi_{i-1, K}\right|_{P_{i-1}}\right),
\end{aligned}
$$

откуда следует требуемое утверждение.

Можно показать, что $P_{K}-$ прямая в $G_{K} \cong \mathbb{P}^{2}$ и $\operatorname{deg}\left(\left.\psi_{i-1, L}\right|_{P_{i-1}}\right)=1$ при $i>K$.

СлЕДСТВИЕ 5.29. Справедливо неравенство $d_{K} \geqslant \sum_{i=K+1}^{N} \nu_{i}^{2}$.

Рассмотрим ограничение функции $P(i)$ на подмножество $[1, K] \subseteq[1, N]$. А именно, пусть $Q:[1, K] \rightarrow \mathbb{N}$ - функция такая, что $Q(i)$ - количество ориентированных путей в графе $\Gamma$, идущих из вершины $G_{N}$ в вершину $G_{i}, i=1, \ldots, K$.

ЗАмЕчАнИЕ 5.30. Справедливо

$$
Q(i)=\sum_{G_{j} \rightarrow G_{i}} Q(j)+\sum_{G_{j} \rightarrow G_{i}}^{j>K} P(j) \geqslant \sum_{G_{j} \rightarrow G_{i}} Q(j) .
$$

ЛЕмма 5.31. Справедливо неравенство

$$
\sum_{i=1}^{K} Q(i) m_{0, i} \geqslant \sum_{i=1}^{K} Q(i) \nu_{i}^{2}+Q(K) d_{K}
$$


ДокАЗАТЕЛЬСТво. При $j=1, \ldots, K$ умножим равенство

$$
\sum_{i=0}^{j-1} m_{i, j}=\nu_{j}^{2}+d_{j}
$$

на $Q(j)$ и просуммируем

$$
\sum_{j=1}^{K} \sum_{i=0}^{j-1} Q(j) m_{i, j}=\sum_{j=1}^{K} Q(j) \nu_{j}^{2}+\sum_{j=1}^{K} Q(j) d_{j},
$$

а затем изменим порядок суммирования

$$
\sum_{j=1}^{K} \sum_{i=0}^{j-1} Q(j) m_{i, j}=\sum_{i=0}^{K-1} \sum_{j=i+1}^{K} Q(j) m_{i, j},
$$

где $m_{i, j} \leqslant d_{i}$ при $1 \leqslant i<j \leqslant K$. Более того, $m_{i, j}>0 \Longleftrightarrow G_{j} \rightarrow G_{i}$. Значит,

$$
\begin{aligned}
\sum_{i=0}^{K-1} \sum_{j=i+1}^{K} Q(j) m_{i, j} & \leqslant \sum_{j=1}^{K} Q(j) m_{0, j}+\sum_{i=1}^{K-1} \sum_{G_{j} \rightarrow G_{i}} Q(j) d_{i} \\
& \leqslant \sum_{j=1}^{K} Q(j) m_{0, j}+\sum_{i=1}^{K-1} Q(i) d_{i},
\end{aligned}
$$

откуда следует неравенство

$$
\sum_{j=1}^{K} Q(j) m_{0, j}+\sum_{i=1}^{K-1} Q(i) d_{i} \geqslant \sum_{j=1}^{K} Q(j) \nu_{j}^{2}+\sum_{j=1}^{K} Q(j) d_{j},
$$

которое, в свою очередь, дает неравенство

$$
\sum_{j=1}^{K} Q(j) m_{0, j} \geqslant \sum_{j=1}^{K} Q(j) \nu_{j}^{2}+Q(K) d_{K}
$$

что является именно тем, что требовалось доказать.

СлеДСТВИЕ 5.32. Справедливо неравенство $\sum_{i=1}^{K} P(i) m_{0, i} \geqslant \sum_{i=1}^{N} P(i) \nu_{i}^{2}$.

Пусть $\Sigma_{0}=\sum_{i=1}^{K} P(i)$ и $\Sigma_{1}=\sum_{i=K+1}^{N} P(i)$. Тогда из элементарного анализа

$$
\sum_{i=1}^{K} P(i) m_{0, i} \geqslant \sum_{i=1}^{N} P(i) \nu_{i}^{2} \geqslant \frac{\left(2 \Sigma_{0}+\Sigma_{1}-a\left(X, \mu \mathscr{D}_{X}, G_{N}\right)\right)^{2}}{\left(\Sigma_{0}+\Sigma_{1}\right)}
$$

поскольку $\sum_{i=1}^{N} P(i) \nu_{i}=2 \Sigma_{0}+\Sigma_{1}-a\left(X, \mu \mathscr{D}_{X}, G_{N}\right)$.

Напомним, что $m_{0, i}=m_{T_{0}}^{i}=m_{Z_{X}}^{i}+m_{C_{\omega}}^{i}$ для $i=1, \ldots, K$ и $m_{C_{\omega}}^{i}=0$ для $i>M$, где натуральное $M \leqslant K$ является наибольшим, удовлетворяющим 
условию $P_{M-1} \in F_{\omega}^{M-1}$, причем выполнено неравенство $\operatorname{mult}_{O}\left(Z_{X}\right) \geqslant m_{Z_{X}}^{i}$ при $i=1, \ldots, K$, а также выполнено аналогичное неравенство $\operatorname{mult}_{O}\left(C_{\omega}\right) \geqslant m_{C_{\omega}}^{i}$ при $i=1, \ldots, M$. Таким образом, получаем

$$
\operatorname{mult}_{O}\left(Z_{X}\right) \Sigma_{0}+\operatorname{mult}_{O}\left(C_{\omega}\right) \Sigma_{0}^{\prime} \geqslant \frac{\left(2 \Sigma_{0}+\Sigma_{1}-a\left(X, \mu \mathscr{D}_{X}, G_{N}\right)\right)^{2}}{\left(\Sigma_{0}+\Sigma_{1}\right)},
$$

где $\Sigma_{0}^{\prime}=\sum_{i=1}^{M} P(i)$. С другой стороны, $\operatorname{mult}_{O}\left(Z_{X}\right) \leqslant F_{\omega} \cdot Z_{X}=2$ и

$$
\operatorname{mult}_{O}\left(C_{\omega}\right) \leqslant-K_{X} \cdot C_{\omega} \leqslant 2-\frac{4 a\left(X, \mu \mathscr{D}_{X}, G_{N}\right)}{b\left(X, F_{\omega}, G_{N}\right)}
$$

и $b\left(X, F_{\omega}, G_{N}\right) \geqslant \sum_{i=1}^{M} q_{i} P(i) \geqslant \Sigma_{0}^{\prime}$, где $q_{i}=$ mult $_{P_{i-1}}\left(F_{\omega}^{i-1}\right)$ для $i=1, \ldots, M$.

Комбинируя все последние неравенства вместе, получаем

$$
2\left(\Sigma_{0}-\Sigma_{0}^{\prime}\right)\left(\Sigma_{0}+\Sigma_{1}\right)+\left(\Sigma_{1}+a\left(X, \mu \mathscr{D}_{X}, G_{N}\right)\right)^{2} \leqslant 0
$$

откуда $\left.a\left(X, \mu \mathscr{D}_{X}, G_{N}\right)\right)=-\Sigma_{1}$ и $\Sigma_{0}=\Sigma_{0}^{\prime}$. Более того, выполнено равенство

$$
\sum_{i=1}^{N} P(i) \nu_{i}^{2}=\frac{\left(2 \Sigma_{0}+\Sigma_{1}-a\left(X, \mu \mathscr{D}_{X}, G_{N}\right)\right)^{2}}{\left(\Sigma_{0}+\Sigma_{1}\right)}
$$

что возможно, только когда $\nu_{1}=\nu_{2}=\cdots=\nu_{N}=\nu$. Следовательно,

$$
\nu\left(\Sigma_{0}+\Sigma_{1}\right)=\sum_{i=1}^{N} P(i) \nu_{i}=2 \Sigma_{0}+\Sigma_{1}-a\left(X, \mu \mathscr{D}_{X}, G_{N}\right)=2\left(\Sigma_{0}+\Sigma_{1}\right)
$$

откуда $\nu=2$.

Таким образом, каждое использованное ранее неравенство обязано быть равенством. В частности, из замечания 5.20 следует, что множество $\mathscr{J}$ состоит из одной точки, что означает, что слой $F_{\omega}$ является единственным слоем расслоения $\tau$, содержащим точки неканоничности лог-пары $\left(X, \mu \mathscr{D}_{X}\right)$. Более того, из равенства

$$
-K_{X} \cdot C_{\omega}=2+4 r=-K_{X} \cdot \sum_{\lambda \in \mathbb{P}^{1}} C_{\lambda}
$$

следует, что все одномерные циклы $C_{\lambda}$ пусты при $\lambda \neq \omega$. Из соотношения

$$
T_{0}=Z_{X}+C_{\omega} \equiv Z+(2+4 r) C
$$

где $Z$ - слой морфизма $\left.f\right|_{E}: E \rightarrow \widetilde{L}$, а $C$ - кривая в $F_{\omega}$ с $-K_{X} \cdot C=1$, следует, что носитель цикла $Z_{X}$ содержится в слоях морфизма $\left.f\right|_{E}$. В частности, точка $O$ содержится в пересечении $F_{\omega} \cap Z_{\delta}$, где $Z_{\delta}$ - слой морфизма $\left.f\right|_{E}: E \rightarrow \widetilde{L}$ над некоторой точкой $\delta$ кривой $\widetilde{L}$. Однако из равенства $2=F_{\omega} \cdot Z_{X}=\operatorname{mult}_{O}\left(Z_{X}\right)$ легко следует, что носитель цикла $Z_{X}$ содержится в слое $Z_{\delta}$, которьй поэтому обязательно должен быть приводимым слоем морфизма $\left.f\right|_{E}$. В частности, точка $\delta$ 
является одной из определенных в $\S 1$ точек $O_{i}$ и $Z_{\delta}=Z_{i}=Z_{i}^{0} \cup Z_{i}^{1}$. С другой стороны, по построению линейная система $\mathscr{D}_{X}$ не составлена из пучка. Значит, существует точка $P \in X$ такая, что $P \notin F_{\omega} \cup E$, а линейная подсистема $\mathscr{D}_{P} \subset \mathscr{D}_{X}$, состоящая из поверхностей в линейной системе $\mathscr{D}_{X}$, проходящих через точку $P$, не имеет неподвижных компонент и, следовательно, $P \in A \cap B$ для двух достаточно обших дивизоров $A$ и $B$ в линейной системе $\mathscr{D}_{P}$. Однако использованные ранее дивизоры $D_{1}$ и $D_{2}$ в линейной системе $\mathscr{D} X$ могут быть заменены на дивизоры $A$ и $B$ соответственно. Следовательно, в теоретико-множественном смысле $P \in A \cap B \subset$ $F_{\omega} \cup E$, что противоречит первоначальному выбору точки $P$. Следовательно, теорема 1.3 доказана полностью.

\section{§6. Доказательство теоремы 1.4}

В обозначениях и предположениях $\S 1$ допустим существование бирационального отображения $\beta: V \rightarrow Y$ такого, что $Y$ - трехмерное многообразие Фано, имеющее произвольные канонические особенности. Основная цель настоящего параграфа состоит в доказательстве следующего результата.

УТВЕРЖДЕНИЕ 6.1. Существует такой бирациональный автоморфизм $\sigma$ многообразия Фано $V$, который является однозначно определенной композицией инволющий Бертини общего слоя расслоения $\tau$ на поверхности дель Пеццо степени, что либо композиция $\beta \circ \sigma$ бирегулярна, либо $\beta \circ \sigma=\alpha \circ \rho_{i, k}$ для некоторого бирегулярного автоморфизма $\alpha$ многообразия Фано $V_{i, k}$.

СЛЕДСТвИЕ 6.2. Справедливо $Y \cong V$ или $Y \cong V_{i, k}$.

СлеДСТвИЕ 6.3. Последовательность $1 \rightarrow \Gamma \rightarrow \operatorname{Bir}(V) \rightarrow \operatorname{Aut}(V) \rightarrow 1$ mочна, где группа $\Gamma$ - свободное произведение инволюиий Бертини общего слоя расслоения $\tau$ на поверхности дель Пецио степени два, который рассматривается как гладкая поверхность дель Пецио степени два с группой Пикара $\mathbb{Z}$, определенная над $\mathbb{C}(x)$.

Пусть $\mathscr{D}_{Y}=\left|-t K_{Y}\right|$ для $t \gg 0$ и $\mathscr{D}_{V}=\beta^{-1}\left(\mathscr{D}_{Y}\right)$. Тогда $\mathscr{D}_{V} \subset\left|-n K_{V}\right|$ для некоторого $n \in \mathbb{N}$. Пусть $\mathscr{D}_{X}-$ собственный прообраз $\mathscr{D}_{V}$ на $X$, а $F$ - слой расслоения $\tau: X \rightarrow \mathbb{P}^{1}$ на поверхности дель Пецщо. Тогда

$$
\mathscr{D}_{X} \sim f^{*}\left(-n K_{V}\right)-m E \sim-n K_{X}-m E \sim f^{*}\left(K_{V}\right) \sim n(F+E)-m E,
$$

где $n>m=\operatorname{mult}_{\widetilde{L}}\left(\mathscr{D}_{V}\right) \geqslant 0$. Пусть $\mu=1 /(n-m)$ и $r=m /(n-m)$. Тогда $K_{X}+\mu \mathscr{D}_{X} \sim_{\mathbb{Q}} r F$.

ЗАмечАниЕ 6.4. Справедливо следующее:

$$
m=0 \quad \Longleftrightarrow \quad r=0 \quad \Longleftrightarrow \quad \mu=\frac{1}{n} \quad \Longleftrightarrow \quad \widetilde{L} \notin \overline{\mathbb{C S}}\left(V, \frac{1}{n} \mathscr{D}_{V}\right)
$$

Лемма 6.5. Рвавенство $\mathbb{C S}\left(X, \mu \mathscr{D}_{X}\right)=\varnothing$ влечет бирегулярность отображения $\beta$. 
ДокаЗАТЕЛЬСТво. Пусть $r>0$. Тогда $\left(X, \varepsilon \mathscr{D}_{X}\right)$ - каноническая модель для некоторого $\varepsilon>\mu$. Значит, $\varkappa\left(Y, \varepsilon \mathscr{D}_{Y}\right)=\varkappa\left(X, \varepsilon \mathscr{D}_{X}\right)=3$ и $\left(Y, \varepsilon \mathscr{D}_{Y}\right)$ также является канонической моделью. Следовательно, отображение $\beta \circ f$ бирегулярно по теореме 2.15, но дивизор $-K_{X}$ не обилен. Значит, $r=0, \mu=\frac{1}{n}$, лог-пара $\left(X, \frac{1}{n} \mathscr{D}_{X}\right)$ является лог-поднятием лог-пары $\left(V, \frac{1}{n} \mathscr{D}_{V}\right)$ и $\widetilde{L} \notin \overline{\mathbb{C}}\left(V, \frac{1}{n} \mathscr{D}_{V}\right)$. В частности, лог-пара $\left(V, \frac{1}{n} \mathscr{D}_{V}\right)$ полутерминальна и $\left(V, \varepsilon \mathscr{D}_{V}\right)$ - каноническая модель для некоторого $\varepsilon \in \mathbb{Q}_{>1 / n}$. В частности, выполнены равенства $\varkappa\left(Y, \varepsilon \mathscr{D}_{Y}\right)=\varkappa\left(V, \varepsilon \mathscr{D}_{V}\right)=3$ и $\left(Y, \varepsilon \mathscr{D}_{Y}\right)$ - каноническая модель. Следовательно, отображение $\beta$ бирегулярно по теореме 2.15 .

Лемма 6.6. Пусть $C \subset X-$ кривая, содержащаяся в слоях расслоения $\tau$, такая, что $C \in \mathbb{C} S\left(X, \mathscr{D}_{X}\right)$. Тогда лог-пара $\left(X, \mu_{X}\right)$ канонична в общей точке кривой $C$.

ДокАЗАТЕЛЬСтво. См. доказательство леммы 5.11.

ЛЕмма 6.7. Существует единственным образом определенная композииия $\sigma$ инволющий Бертини общего слоя расслоения $\tau$ такая, что лог-пара $\left(X, \mu_{\sigma} \sigma\left(\mathscr{D}_{X}\right)\right)$ имеет канонические особенности в общих точках кривьх многообразия $X$, где $\mu_{\sigma} \in \mathbb{Q}>0$ задано соотношением $K_{X}+\mu_{\sigma} \sigma\left(\mathscr{D}_{X}\right) \sim_{\mathbb{Q}} r_{\sigma} F$ для некоторого неотрицательного рационального числа $r_{\sigma}$.

ДокАЗАТЕЛЬСтво. См. доказательство леммы 5.12.

ЗАмЕчАниЕ 6.8. Для доказательства утверждения 6.1 бирациональное отображение $\beta$ можно заменить на композицию $\beta \circ \sigma^{-1}$ и считать, что лог-пара $\left(X, \mathscr{D}_{X}\right)$ имеет канонические особенности в общих точках кривых на многообразии $X$.

Лемма 6.9. Предположим, что лог-пара $\left(X, \mu_{X}\right)$ канонична. Тогда $r=0$.

ДоказАТЕЛьство. Пусть $r>0$. Тогда $\varkappa\left(X, \mu \mathscr{D}_{X}\right)=1$, но $\varkappa\left(Y, \mu \mathscr{D}_{Y}\right) \in$ $\{-\infty, 0,3\}$ по построению линейной системы $\mathscr{D}_{Y}$, что противоречит бирациональной инвариантности размерности Кодаиры подвижной лог-пары.

Пусть $h: U \rightarrow X-$ бирациональный морфизм, $U$ неособо, $\beta \circ f \circ h$ регулярно,

$$
K_{U}+\mu \mathscr{D}_{U} \sim_{\mathbb{Q}} h^{*}(r F)+\sum_{i=1}^{k} a_{i} E_{i}
$$

где $\mathscr{D}_{U}=h^{-1}\left(\mathscr{D}_{X}\right), E_{i}-h$-исключительный дивизор и $a_{i} \in \mathbb{Q}$. Определим подмножество $\mathscr{J} \subset \mathbb{P}^{1}$ как образ исключительных дивизоров $E_{i}$ с $a_{i}<0$. Для $\lambda \in \mathscr{J}$ положим

$$
h^{*}\left(F_{\lambda}\right) \sim h^{-1}\left(F_{\lambda}\right)+\sum_{j=1}^{k_{\lambda}} b_{j} E_{j},
$$

где $F_{\lambda}$ - слой расслоения $\tau$ над точкой $\lambda$ и $b_{i} \in \mathbb{N}$. Наконец, положим $\mathscr{I}=$ $\bigcup_{\lambda \in \mathscr{J}} \mathscr{I}_{\lambda}$, а для $\lambda \in \mathscr{J}$ определим множество $\mathscr{I}_{\lambda} \subset\{1, \ldots, k\}$ следующим образом: $i \in \mathscr{I}_{\lambda}$ в том и только том случае, если $h\left(E_{i}\right)$ является точкой в слое $F_{\lambda}$ и $a_{i}<0$. 
УТВЕРЖДЕНИЕ 6.10. Справедливо неравенство

$$
r+\sum_{\lambda \in \mathscr{J}} \min \left\{\frac{a_{i}}{b_{i}} \mid h\left(E_{i}\right) \in F_{\lambda} \quad u \quad a_{i}<0\right\} \leqslant 0 .
$$

ДокАЗАТЕЛЬСтво. Предположим, что утверждение, которое требуется доказать, неверно. В этом случае сушествуют положительные рациональные числа $\varepsilon$ и $c_{\lambda}$ такие, что $r=\varepsilon+\sum_{\lambda \in \mathscr{J}} c_{\lambda}$ и $c_{\lambda}+\min \left\{a_{i} / b_{i} \mid h\left(E_{i}\right) \in F_{\lambda}\right.$ и $\left.a_{i}<0\right\}>0$. Тогда

$$
K_{U}+\mu \mathscr{D}_{U} \sim_{\mathbb{Q}} h^{*}(\varepsilon F)+\sum_{\lambda \in \mathscr{J}}\left(h^{*}\left(c_{\lambda} F_{\lambda}\right)+\sum_{i \in \mathscr{I}_{\lambda}} a_{i} E_{i}\right)+\sum_{i \notin \mathscr{I}} a_{i} E_{i},
$$

но для всех $\lambda \in \mathscr{J}$ дивизор $h^{*}\left(c_{\lambda} F_{\lambda}\right)+\sum_{i \in \mathscr{I}_{\lambda}} a_{i} E_{i}$ эффективен в силу выбора числа $c_{\lambda}$, а дивизор $\sum_{i \notin \mathscr{I}} a_{i} E_{i}$ эффективен, потому что лог-пара $\left(X, \mu \mathscr{D}_{X}\right)$ канонична в обших точках кривых на $X$. Значит, $\varkappa\left(U, \mu \mathscr{D}_{U}\right)=1$, но $\varkappa\left(Y, \mu \mathscr{D}_{Y}\right) \in\{-\infty, 0,3\}$ по построению, что противоречит бирациональной инвариантности размерности Кодаиры.

Лемма 6.11. Справедливо равенство $r=0$.

ДоказАТЕльСтво. Пусть $Z$ - слой морфизма $\left.f\right|_{E}: E \rightarrow \widetilde{L}, C$-кривая в слоях расслоения $\tau \mathrm{c}-K_{X} \cdot C=1$, а $D_{1}$ и $D_{2}$ - достаточно общие дивизоры в линейной системе $\mathscr{D}_{X}$. Тогда $\overline{\mathbb{N}}(X)=\mathbb{R}_{\geqslant 0} Z \oplus \mathbb{R}_{\geqslant 0} C$ и

$$
\mu^{2} D_{1} \cdot D_{2}=Z_{X}+\sum_{\lambda \in \mathbb{P}^{1}} C_{\lambda} \equiv Z+(2+4 r) C,
$$

где все компоненты эффективного одномерного цикла $Z_{X}$ не содержатся в слоях расслоения $\tau$, а каждая компонента эффективного одномерного цикла $C_{\lambda}$ содержится в слое $F_{\lambda}$ расслоения $\tau$ над точкой $\lambda \in \mathbb{P}^{1}$. Таким образом,

$$
-K_{X} \cdot \sum_{\lambda \in \mathbb{P}^{1}} C_{\lambda}=2+4 r \leqslant 2-4 \sum_{\lambda \in \mathscr{J}} \min \left\{\frac{a_{i}}{b_{i}} \mid h\left(E_{i}\right) \in F_{\lambda} \text { и } a_{i}<0\right\}
$$

по утверждению 6.10 . Пусть $r>0$. Тогда существует точка $\omega \in \mathbb{P}^{1}$ такая, что

$$
-K_{X} \cdot C_{\omega} \leqslant 2-4 \frac{a_{t}}{b_{t}}
$$

где $a_{t} / b_{t}=\min \left\{a_{i} / b_{i} \mid h\left(E_{i}\right) \in F_{\omega}\right.$ и $\left.a_{i}<0\right\}$. Теперь можно слово в слово повторить доказательство теоремы 1.3 , начиная с леммы 5.22 , и получить противоречие.

СледСтвиЕ 6.12. Справедливо следующее: $\widetilde{L} \notin \overline{\mathbb{C S}}\left(V, \frac{1}{n} \mathscr{D}_{V}\right)$.

Лемма 6.13. Равенство $\overline{\mathbb{C S}}\left(V, \frac{1}{n} \mathscr{D}_{V}\right)=\varnothing$ влечет бирегулярность отображения $\beta$. 
ДокАЗАТЕльство. Пусть $\overline{\mathbb{C S}}\left(V, \frac{1}{n} \mathscr{D}_{V}\right)=\varnothing$. Тогда $\left(V, \varepsilon \mathscr{D}_{V}\right)-$ каноническая модель для некоторого $\varepsilon \in \mathbb{Q}_{>1 / n}$ и $\varkappa\left(Y, \varepsilon \mathscr{D}_{Y}\right)=\varkappa\left(V, \varepsilon \mathscr{D}_{V}\right)=3$, откуда $\left(Y, \varepsilon \mathscr{D}_{Y}\right)-$ каноническая модель. Отображение $\beta$ бирегулярно по теореме 2.15 .

ЗАмечание 6.14. Линейная система $\mathscr{D}_{V}$ не содержится в слоях доминантного отображения $\chi: V \rightarrow Z$ такого, что $Z-$ кривая или поверхность.

Лемма 6.15. Множество $\overline{\mathbb{C S}}\left(V, \frac{1}{n} \mathscr{D}_{V}\right)$ не содержит кривых и гладких точек на $V$.

ДокАЗАТЕЛЬСТво. См. доказательство лемм 5.3-5.6.

Лемма 6.16. Множество $\overline{\mathbb{C S}}\left(V, \frac{1}{n} \mathscr{D}_{V}\right)$ не содержит точек, отличных om $O_{i}$.

ДокАЗАТЕЛЬСТво. Допустим, что множество $\overline{\mathbb{C S}}\left(V, \frac{1}{n} \mathscr{D}_{V}\right)$ содержит точку $O$ многообразия $V$ такую, что $O \neq O_{i}$. Тогда $O$ содержится в кривой $\widetilde{L}$ по лемме 6.15 , но из равенства $r=0$ следует, что $\widetilde{L} \notin \overline{\mathbb{C S}}\left(V, \frac{1}{n} \mathscr{D}_{V}\right)$. Значит, $\mathbb{C S}\left(X, \frac{1}{n} \mathscr{D}_{X}\right)$ содержит либо неприводимый слой $Z$ морфизма $\left.f\right|_{E}: E \rightarrow \widetilde{L}$ над $O$, либо некоторую точку $P \in Z$.

Предположим, что $\mathbb{C S}\left(X, \frac{1}{n} \mathscr{D}_{X}\right)$ содержит кривую $Z$. Пусть $g: W \rightarrow X-$ раздутие кривой $Z, \mathscr{D}_{W}$ - собственный прообраз $\mathscr{D}_{X}$ на $W$, а $G$ - исключительный дивизор раздутия $g$. Тогда линейная система $\left|-K_{W}\right|$ свободна, морфизм $\varphi_{\left|-K_{W}\right|}$ является расслоением на эллиптические кривые и $\mathscr{D}_{W} \cdot C=2 n-\operatorname{mult}_{Z}\left(\mathscr{D}_{X}\right)$ для достаточно общего слоя $C$ расслоения $\varphi_{\left|-K_{W}\right|}$. Следовательно, линейная система $\mathscr{D}_{W}$ содержится в слоях расслоения $\varphi_{\left|-K_{W}\right|}$, что невозможно по построению.

Предположим теперь, что $\mathbb{C S}\left(X, \frac{1}{n} \mathscr{D}_{X}\right)$ содержит некоторую точку $P \in Z$. Тогда

$$
\mathscr{D}_{X}^{2}=\operatorname{mult}_{Z}\left(\mathscr{D}_{X}^{2}\right) Z+C_{X}
$$

где носитель одномерного цикла $C_{X}$ не содержит кривой $Z$, и

$$
\operatorname{mult}_{P}\left(\mathscr{D}_{X}^{2}\right)=\operatorname{mult}_{Z}\left(\mathscr{D}_{X}^{2}\right)+\operatorname{mult}_{P}\left(C_{X}\right) \geqslant 4 n^{2}
$$

по теореме 3.12. С другой стороны, $Z \cdot F=2$ и $\mathscr{D}_{X}^{2} \cdot F=2 n^{2}$, где $F$ - слой расслоения $\tau$ на поверхности дель Пещю степени два. В частности, $\operatorname{mult}_{Z}\left(\mathscr{D}_{X}^{2}\right) \leqslant n^{2}$ и выполнено неравенство $\operatorname{mult}_{P}\left(C_{X}\right) \geqslant 3 n^{2}$. Пусть $H$ - общий дивизор в линейной системе $\left|-K_{X}\right|$, содержаший кривую $Z$. Тогда $H$ не содержит неприводимых компонент цикла $C_{X}$ и

$$
n^{2}=H \cdot C_{X} \geqslant \operatorname{mult}_{P}\left(C_{X}\right) \geqslant 3 n^{2},
$$

что доказывает требуемое утверждение.

ЛЕмма 6.17. Пусть $O_{i} \in \mathbb{C S}\left(V, \frac{1}{n} \mathscr{D}_{V}\right)$. Тогда $\beta=\alpha \circ \rho_{i, 0}$ или $\beta=\alpha \circ \rho_{i, 1}$ для некоторого бирегулярного автоморфизма $\alpha$ многообразия $V_{i, 0}$ или $V_{i, 1}$ соответственно. 
ДоказАТЕЛЬСтво. Можно считать, что $i=1$. Множество $\mathbb{C} S\left(X, \frac{1}{n} \mathscr{D}_{X}\right)$ содержит либо некоторую точку $P \in Z_{1}$, либо неприводимую компоненту слоя $Z_{1}$, где по определению $Z_{1}=Z_{1}^{0} \cup Z_{1}^{1}=f^{-1}\left(O_{1}\right)$. Более того, практически дословное повторение части доказательства леммы 5.13 показывает, что первый случай невозможен, а также, что множество $\mathbb{C} S\left(X, \frac{1}{n} \mathscr{D}_{X}\right)$ состоит из одной неприводимой компоненты слоя $Z_{1}$. Можно считать, что $\mathbb{C} S\left(X, \frac{1}{n} \mathscr{D}_{X}\right)=\left\{Z_{1}^{0}\right\}$. Пусть $g: W \rightarrow X$ - раздутие гладкой кривой $Z_{1}^{0}$, а $\mathscr{D}_{W}-$ собственный прообраз $\mathscr{D}_{X}$ на $W$. Теперь практически дословное повторение еше одной части доказательства леммы 5.13 показывает, что лог-пара $\left(W, \frac{1}{n} \mathscr{D}_{W}\right)$ имеет терминальные особенности. Докажем, что $\beta=\alpha \circ \rho_{1,0}$ для некоторого бирегулярного автоморфизма $\alpha$ особого многообразия Фано $V_{1,0}$.

В обозначениях $\S 4$ рассмотрим антифлип $\check{h} \circ \check{p} \circ \widehat{p}^{-1} \circ h^{-1}: W \rightarrow \breve{W}$ в $Z_{1}^{1}$ и собственный прообраз $\mathscr{D}_{\breve{W}}$ линейной системы $\mathscr{D}_{W}$ на $\breve{W}$. Особенности $\left(\check{W}, \frac{1}{n} \mathscr{D}_{\breve{W}}\right)$ терминальны, поскольку $\left(K_{W}+\frac{1}{n} \mathscr{D}_{W}\right) \cdot Z_{1}^{1}=0$ и $\check{h} \circ \check{p} \circ \widehat{p}^{-1} \circ h^{-1}$ является лог-ф̆лопом для $\left(W, \frac{1}{n} \mathscr{D}_{W}\right)$, для некоторого $\varepsilon \in \mathbb{Q}_{>1 / n}$ лог-пара $\left(\breve{W}, \varepsilon \mathscr{D}_{\breve{W}}\right)$ также терминальна и

$$
K_{\breve{W}}+\varepsilon \mathscr{D}_{\breve{W}} \sim_{\mathbb{Q}}\left(\frac{1}{n}-\varepsilon\right) K_{\breve{W}},
$$

где дивизор $-K_{\breve{W}}$ численно эффективен и объемен по лемме 4.6 , а для $n \gg 0$ линейная система $\left|-n K_{\breve{W}}\right|$ задает бирациональный морфизм $\varphi_{\mid-n K_{\breve{W}}}: \check{W} \rightarrow V_{1,0}$, который стягивает кривые, имеюшие тривиальноепересечение с $-K_{\breve{W}}$. В частности, отсюда следует, что лог-пара $\left(V_{1,0}, \varepsilon \mathscr{D}_{V_{1,0}}\right)$ является канонической моделью и выполнены равенства $\varkappa\left(V_{1,0}, \varepsilon \mathscr{D}_{V_{1,0}}\right)=\varkappa\left(Y, \varepsilon \mathscr{D}_{Y}\right)=3$. Значит, лог-пара $\left(Y, \varepsilon \mathscr{D}_{Y}\right)$ также является канонической моделью. С другой стороны, $\mathscr{D}_{V_{1,0}}=\rho_{1,0}\left(\mathscr{D}_{V}\right)$. Следовательно, бирациональное отображение $\rho_{1,0} \circ \beta^{-1}$ бирегулярно по теореме 2.15 .

Таким образом, теорема 1.4 доказана полностью.

\section{§7. Доказательство теоремы 1.15}

В обозначениях и предположениях $\S 1$ допустим, что поверхность $E \subset X$ неособа, прямая $L$ является единственной прямой на секстике $S$, которая проходит через одну из точек $\gamma\left(O_{i}\right) \in \mathbb{P}^{3}$, существуют бирациональное отображение $\beta: V \rightarrow Y$ и расслоение $\pi: Y \rightarrow \mathbb{P}^{1}$ такие, что общий слой расслоения $\pi$ является гладкой связной поверхностью с численно тривиальным каноническим дивизором. В настоящем параграфе будет построен бирациональный автоморфизм $\sigma$ многообразия $V$ и найден пучок $\mathscr{P}$ в системе $\left|-K_{V}\right|$ такие, что $\rho \circ \sigma=\varphi \mathscr{P}$, где $\rho=\pi \circ \beta$.

ЗАмЕчАнИЕ 7.1. Некоторые аргументы, которые эффективно использовались в $\S \S 5,6$, к сожалению уже не будут верны в предположениях настояшего параграpa.

Пусть $\mathscr{D}_{V}=\beta^{-1}\left(\left|\tau^{*}\left(\mathscr{O}_{\mathbb{P}^{1}}(1)\right)\right|\right)$. Тогда $\mathscr{D}_{V} \subset\left|-n K_{V}\right|$ для некоторого $n \in \mathbb{N}$.

ЛЕмма 7.2. Справедливо следующее: $\overline{\mathbb{C S}}\left(V, \frac{1}{n} \mathscr{D}_{V}\right) \neq \varnothing$.

ДоказАТЕЛЬСТво. Пусть $\overline{\mathbb{C S}}\left(V, \frac{1}{n} \mathscr{D}_{V}\right)=\varnothing$. Тогда $\varkappa\left(V, \varepsilon \mathscr{D}_{V}\right)=3, \varepsilon \in \mathbb{Q}_{>1 / n}$, но по построению линейной системы $\mathscr{D}_{V}$ вьполнено неравенство $\varkappa\left(V, \varepsilon \mathscr{D}_{V}\right) \leqslant 1$. 
Лемма 7.3. Множество $\mathbb{C S}\left(V, \frac{1}{n} \mathscr{D}_{V}\right)$ не содержит гладких точек многообразия $V$.

ДокАЗАТЕльСтво. Пусть $O \in \mathbb{C} S\left(V, \frac{1}{n} \mathscr{D}_{V}\right)$ и $O \notin \widetilde{L}$ - точка, а $H_{O} \in\left|-K_{V}\right|$ общая поверхность, содержашая точку $O$. Тогда $2 n^{2}=H_{O} \cdot \mathscr{D}_{V}^{2} \geqslant 4 n^{2}$ по теореме 3.12 .

Лемма 7.4. Пусть $\mathbb{C S}\left(V, \frac{1}{n} \mathscr{D}_{V}\right)$ содержит кривую $C \neq \widetilde{L} . \quad$ Тогда $\gamma(C)-$ прямая.

ДоказАТЕЛЬСтво. Неравенство $\operatorname{mult}_{C}\left(\mathscr{D}_{V}\right) \geqslant n$ равносильно утверждению, что кривая $C$ содержится в множестве $\mathbb{C}\left(V, \frac{1}{n} \mathscr{D}_{V}\right)$. Следовательно, для достаточно обшего дивизора $H$ в линейной системе $\left|-K_{V}\right|$ из неравенств

$$
2 n^{2}=H \cdot \mathscr{D}_{V}^{2} \geqslant \operatorname{mult}_{C}\left(\mathscr{D}_{V}^{2}\right) H \cdot C \geqslant \operatorname{mult}_{C}^{2}\left(\mathscr{D}_{V}\right) H \cdot C \geqslant n^{2} H \cdot C
$$

следует неравенство $-K_{V} \cdot C \leqslant 2$. В случае, когда выполнено $-K_{V} \cdot C=1$, кривая $\gamma(C)$ является прямой в $\mathbb{P}^{3}$. Таким образом, можно считать, что выполнены равенства $-K_{V} \cdot C=2, \operatorname{mult}_{C}\left(\mathscr{D}_{V}\right)=n$ и $_{\text {mult }}\left(\mathscr{D}_{V}^{2}\right)=n^{2}$, кривая $\gamma(C)$ является коникой в $\mathbb{P}^{3}$, отображение $\left.\gamma\right|_{C}: C \rightarrow \gamma(C)$ является изоморфизмом, а носитель эффективного одномерного цикла $\mathscr{D}_{V}^{2}$ целиком состоит из кривой $C$.

Предположим сначала, что $C \cap \widetilde{L}=\varnothing$. Пусть в этом случае $g: W \rightarrow V-$ раздутие кривой $C, G$ - исключительньй дивизор раздутия $g$, а $\mathscr{D}_{W}-$ собственный прообраз линейной системы $\mathscr{D}_{V}$ на трехмерном многообразии $W$. Покажем, что эффективный дивизор $g^{*}\left(-3 K_{V}\right)-G$ численно эффективен. Допустим, что $\gamma(C) \not \subset S$. Рассмотрим кривую $\widetilde{C} \subset W$ такую, что $\gamma \circ g(\widetilde{C})=\gamma(C)$ и $g(\widetilde{C}) \neq C$. Кривая $\widetilde{C}$ является единственной кривой в базисном множестве линейной системы $\left|g^{*}\left(-2 K_{V}\right)-G\right|$ и выполнено равенство $\left(g^{*}\left(-2 K_{V}\right)-G\right) \cdot \widetilde{C}=-2$, откуда следует, что дивизор $g^{*}\left(-3 K_{V}\right)-E$ численно эффективен. Теперь предположим, что $\gamma(C) \subset S$. В этом случае базисное множество линейной системы $\left|g^{*}\left(-2 K_{V}\right)-G\right|$ содержится в $G$. Пусть $s_{\infty}-$ исключительное сечение линейчатой поверхности $\left.g\right|_{G}: G \rightarrow C$. Легко видеть, что численная эффективность дивизора $g^{*}\left(-3 K_{V}\right)-G$ следует в этом случае из выполнения неравенства $\left.\left(g^{*}\left(-3 K_{V}\right)-G\right)\right|_{G} \cdot s_{\infty} \geqslant 0$, но элементарные вычисления показывают, что

$$
\left.\left(g^{*}\left(-3 K_{V}\right)-G\right)\right|_{G} \cdot s_{\infty}=6+\frac{s_{\infty}^{2}}{2}
$$

и $G^{3}=0$. Покажем, что $s_{\infty}^{2} \geqslant-12$. Пусть $\mathscr{N}_{C / V} \cong \mathscr{O}_{C}(m) \oplus \mathscr{O}_{C}(n)$ для $m \geqslant n$. Тогда

$$
m+n=\operatorname{deg}\left(\mathscr{N}_{C / V}\right)=-K_{V} \cdot C+2 g(C)-2=0
$$

и точная последовательность

$$
\left.0 \rightarrow \mathscr{N}_{C / \widetilde{S}} \rightarrow \mathscr{N}_{C / V} \rightarrow \mathscr{N}_{\widetilde{S} / V}\right|_{C} \rightarrow 0
$$


влечет $n \geqslant \operatorname{deg}\left(\mathscr{N}_{C / \widetilde{S}}\right)=-6$, где $\widetilde{S}=\gamma^{-1}(S)$. Следовательно, $s_{\infty}^{2}=n-m=$ $2 n \geqslant-12$ и дивизор $g^{*}\left(-3 K_{V}\right)-G$ численно эффективен. В частности, выполнено неравенство

$$
6 n^{2}-\operatorname{mult}_{C}\left(\mathscr{D}_{V}\right)\left(6 \operatorname{mult}_{C}\left(\mathscr{D}_{V}\right)+4 n\right)=\left(g^{*}\left(-3 K_{V}\right)-G\right) \cdot \mathscr{D}_{W}^{2} \geqslant 0,
$$

откуда следует противоречие. Значит, $C \cap \widetilde{L} \neq \varnothing$.

Пусть $\widehat{C}=f^{-1}(C)$ и $h: U \rightarrow X-$ раздутие кривой $C$, пусть $H$ - исключительный дивизор раздутия $h, \mathscr{D}_{U}-$ собственный прообраз $\mathscr{D}_{V}$ на $U, D_{1}$ и $D_{2}-$ два достаточно общих дивизора в $\mathscr{D}_{U}, t=E \cdot \widehat{C}=2-F \cdot \widehat{C}$, где $F$ - слой расслоения $\tau$. Тогда

$$
T_{U}=D_{1} \cdot D_{2}=\sum_{O_{i} \neq \delta \in \tilde{L} \cap C} p_{\delta} Z_{\delta}+\sum_{O_{i} \in \tilde{L} \cap C}\left(p_{i, 0} \bar{Z}_{i}^{0}+p_{i, 1} \bar{Z}_{i}^{1}\right)+C_{H},
$$

где $Z_{\delta}$ - собственньй прообраз на многообразии $U$ неприводимого слоя бирационального морфизма $f$ над $\delta \in \widetilde{L}, \bar{Z}_{i}^{k}$ - собственный прообраз на $U$ компоненты $Z_{i}^{k}$ приводимого слоя $Z_{i}=Z_{i}^{0} \cup Z_{i}^{1}$ бирационального морфизма $f$ над $O_{i}$, целые числа $p_{\delta}$ и $p_{i, k}$ неотрицательны, носитель цикла $C_{H}$ содержится в $H$. Более того,

$$
(2-t) n^{2}=h^{*}(F) \cdot \mathscr{D}_{U}^{2}=\sum 2 p_{\delta}+\sum p_{i, 0}+\sum p_{i, 1},
$$

поскольку одномерный цикл $C_{H}$ либо пуст, либо стягивается морфизмом $h$ ввиду равенства $\operatorname{mult}_{C}^{2}\left(\mathscr{D}_{V}\right)=\operatorname{mult}_{C}\left(\mathscr{D}_{V}^{2}\right)$. Легко видеть, что дивизор $h^{*}\left(-3 K_{X}\right)-H$ не является численно эффективным. Тем не менее всегда выполнены неравенства

$\left(h^{*}\left(-3 K_{X}\right)-H\right) \cdot T_{U} \geqslant-H \cdot C_{H}-\sum p_{\delta}-\sum p_{i, 0}-\sum p_{i, 1} \geqslant(t-2) n^{2} \geqslant-n^{2}$

и, следовательно, выполнено

$$
6 n^{2}-\operatorname{mult}_{C}\left(\mathscr{D}_{V}\right)\left(6 \operatorname{mult}_{C}\left(\mathscr{D}_{V}\right)+4 n\right)=\left(h^{*}\left(-3 K_{X}\right)-H\right) \cdot T_{U} \geqslant-n^{2},
$$

откуда следует противоречие, которое доказьвает необходимое утверждение.

Лемма 7.5. Пусть $\mathbb{C S}\left(V, \frac{1}{n} \mathscr{D}_{V}\right)$ содержит кривую $C u-K_{V} \cdot C=2$. Тогда существует пучок $\mathscr{P}$ поверхностей $\mathrm{K} 3$ в линейной системе $\left|-K_{V}\right|$ такой, что $\rho=\varphi \mathscr{P}$.

ДокаЗАТЕЛЬСтво. Образ кривой $C$ на $\mathbb{P}^{3}$ является прямой по лемме 7.4. Следовательно, сушествует пучок $\mathscr{P} \subset\left|-K_{V}\right|$, состоящий из поверхностей, которые содержат кривую $C$. Разрешим неопределенности рационального отображения $\varphi_{\mathscr{P}}$ посредством бирационального морфизма $g: W \rightarrow V$ такого, что $W$ - гладкое и существует единственный $g$-исключительный дивизор $G$ на многообразии $W$, который доминирует кривую $C$. Пусть $\mathscr{D}_{W}-$ собственный прообраз $\mathscr{D}_{V}$ на $W$, а $D_{W}$ - общий дивизор в слоях расслоения $\varphi \mathscr{P} \circ g: W \rightarrow \mathbb{P}^{1}$. Тогда равенство $\operatorname{mult}_{C}\left(\mathscr{D}_{V}\right)=n$ влечет

$$
\left.\mathscr{D}_{W}\right|_{D_{W}} \sim \sum_{i=1}^{k} a_{i} G_{i},
$$

где $G_{i}$ - исключительные дивизоры морфизма $g$, которые отображаются в точки на многообразии $V$, а $a_{i}$ - целые числа. С другой стороны, линейная система $\mathscr{D}_{W}$ не имеет неподвижных компонент. Значит, $\varphi_{\mathscr{D}_{W}}=\varphi \mathscr{P} \circ \mathrm{g}$ и $\rho=\varphi \mathscr{P}$. 
Лемма 7.6. Пусть $\mathbb{C S}\left(V, \frac{1}{n} \mathscr{D}_{V}\right)$ содержит кривую $C$ и $C \cap \widetilde{L}=\varnothing$. Тогда существует пучок $\mathscr{P}$ поверхностей $\mathrm{K} 3$ в линейной системе $\left|-K_{V}\right|$ такой, что $\rho=\varphi \mathscr{P}$.

ДокАЗАТЕЛЬСТво. См. доказательство леммы 5.5.

ЛЕмма 7.7. Предположим, ито $\overline{\mathbb{C S}}\left(V, \frac{1}{n} \mathscr{D}_{V}\right)$ не содержит кривую $\widetilde{L}$, но содержит некоторую кривую $C$ на $V$. Тогда существует пучок $\mathscr{P} \subset\left|-K_{V}\right|$ такой, что $\rho=\varphi \mathscr{P}$.

ДокАЗАТЕЛЬСТво. См. доказательство леммы 5.6.

Лемма 7.8. Множество $\overline{\mathbb{C S}}\left(V, \frac{1}{n} \mathscr{D}_{V}\right)$ содержит либо кривую на $V$, либо точку $O_{i}$.

ДокАЗАТЕЛЬСТво. Предположим, что утверждение, которое требуется доказать, неверно. В этом случае из лемм 7.3-7.7 следует сушествование точки $O \neq O_{i}$ такой, что $O \in \widetilde{L}$ и $O \in \overline{\mathbb{C S}}\left(V, \frac{1}{n} \mathscr{D}_{V}\right)$. Собственный прообраз $\mathscr{D}_{X}$ линейной системы $\mathscr{D}_{V}$ на многообразии $X$ является линейной подсистемой линейной системы $\left|-n K_{X}\right|$, а множество $\mathbb{C} S\left(X, \frac{1}{n} \mathscr{D}_{X}\right)$ содержит либо гладкий неприводимый слой $Z$ бирационального морфизма $f$ над точкой $O$, либо некоторую точку $P \in Z$.

Пусть $\mathbb{C S}\left(X, \frac{1}{n} \mathscr{D}_{X}\right)$ содержит точку $P$ на $Z$. Тогда $\mathscr{D}_{X}^{2}=\operatorname{mult}_{Z}\left(\mathscr{D}_{X}^{2}\right) Z+C_{X}$, где носитель одномерного эффективного цикла $C_{X}$ не содержит кривую $Z$ и

$$
\operatorname{mult}_{P}\left(\mathscr{D}_{X}^{2}\right)=\operatorname{mult}_{Z}\left(\mathscr{D}_{X}^{2}\right)+\operatorname{mult}_{P}\left(C_{X}\right) \geqslant 4 n^{2}
$$

по теореме 3.12. С другой стороны, $Z \cdot F=2$ и $\mathscr{D}_{X}^{2} \cdot F=2 n^{2}$, где $F$ - слой расслоения $\tau$ на поверхности дель Пеццо. В частности, mult ${ }_{Z}\left(\mathscr{D}_{X}^{2}\right) \leqslant n^{2}, \operatorname{mult}_{P}\left(C_{X}\right) \geqslant$ $3 n^{2}$ и

$$
2 n^{2}=H \cdot C_{X} \geqslant \operatorname{mult}_{P}\left(C_{X}\right) \geqslant 3 n^{2},
$$

где $H$ - обшая поверхность в $\left|-K_{X}\right|$, проходящая через кривую $Z$.

Пусть $Z \in \mathbb{C S}\left(X, \frac{1}{n} \mathscr{D}_{X}\right), g: W \rightarrow X$ - раздутие кривой $Z, \mathscr{D}_{W}$ - собственный прообраз линейной системы $\mathscr{D}_{X}$ на многообразии $W$, а $G$ - исключительный дивизор раздутия $g$. Тогда линейная система $\left|-K_{W}\right|$ свободна, а морфизм $\varphi_{\mid-K_{W}} \mid$ является эллиптическим расслоением. Для обшего слоя $C$ расслоения $\varphi\left|-K_{W}\right|$ вьполнено численное равенство $\mathscr{D}_{W} \cdot C=2 n-\operatorname{mult}_{Z}\left(\mathscr{D}_{X}\right)$, откуда следует, что $\mathscr{D}_{W}$ содержится в слоях расслоения $\varphi_{\left|-K_{W}\right|}$. Более того, множество $\mathbb{C} \mathbb{S}\left(W, \frac{1}{n} \mathscr{D}_{W}\right)$ не содержит кривых, которые не стягиваются морфизмом $\tau \circ g$, поскольку в противном случае

$$
2 n^{2}=\mathscr{D}_{X}^{2} \cdot F \geqslant 3 n^{2},
$$

где $F$ - слой расслоения $\tau$. Ранее было показано, что $\mathbb{C} S\left(X, \frac{1}{n} \mathscr{D}_{X}\right)$ не содержит точек в неприводимых слоях морфизма $f$. Пусть $\mathbb{C S}\left(X, \frac{1}{n} \mathscr{D}_{X}\right)$ содержит точку $P_{i}$, содержашуюся в приводимом слое $Z_{i}=Z_{i}^{0} \cup Z_{i}^{1}$ морфизма $f, H_{Z_{i}}$-обший дивизор в линейной системе $\left|-K_{X}\right|$, которьй содержит слой $Z_{i}$, и

$$
n^{2}\left(Z_{i}+2 C\right) \equiv D_{1} \cdot D_{2}=\operatorname{mult}_{Z_{i}^{0}}\left(\mathscr{D}_{X}^{2}\right) Z_{i}^{0}+\operatorname{mult}_{Z_{i}^{1}}\left(\mathscr{D}_{X}^{2}\right) Z_{i}^{1}+C_{X}+R_{X}
$$


где $D_{1}$ и $D_{2}$ - общие дивизоры в линейной системе $\mathscr{D}_{X}, C_{X}$ - эффективный одномерньй цикл на $X$, чьи компоненты содержатся в слоях расслоения $\tau, R_{X}$ - эффективный одномерный цикл на многообразии $X$, чьи компоненты не содержатся в слоях расслоения $\tau, C$ - такая кривая в слоях расслоения $\tau$, что $-K_{X} \cdot C=1$. Тогда

$$
2 n^{2}=H_{Z_{i}} \cdot\left(C_{X}+R_{X}\right) \geqslant \operatorname{mult}_{P}\left(C_{X}\right)+\operatorname{mult}_{P}\left(R_{X}\right)>2 n^{2},
$$

так как mult $P_{i}\left(D_{1} \cdot D_{2}\right) \geqslant 4 n^{2}$ по теореме 3.12 , выполнено равенство

$$
\text { mult }_{Z_{i}^{0}}\left(\mathscr{D}_{X}^{2}\right)+\text { mult }_{Z_{i}^{1}}\left(\mathscr{D}_{X}^{2}\right)+R_{X} \cdot F=2 n^{2}
$$

и $R_{X} \neq \varnothing$. Таким образом, $\mathbb{C} \mathbb{S}\left(W, \frac{1}{n} \mathscr{D}_{W}\right)=\varnothing$ и $\varkappa\left(W, \varepsilon \mathscr{D}_{W}\right)=2$ для некоторого рационального $\varepsilon$, большего $\frac{1}{n}$, но $\varkappa\left(W, \varepsilon \mathscr{D}_{W}\right) \leqslant 1$ по построению линейной системы $\mathscr{D}_{V}$.

Пусть $\mathscr{D}_{X}-$ собственньй прообраз $\mathscr{D}_{V}$ на $X$, а $F$ - слой расслоения $\tau$. Тогда

$$
\mathscr{D}_{X} \sim f^{*}\left(-n K_{V}\right)-m E \sim-n K_{X}-m E \sim f^{*}\left(K_{V}\right) \sim n(F+E)-m E,
$$

где $n>m=\operatorname{mult}_{\widetilde{L}}\left(\mathscr{D}_{V}\right)$. Пусть $\mu=1 /(n-m)$ и $r=m /(n-m)$. Тогда $K_{X}+$ $\mu \mathscr{D}_{X} \sim_{\mathbb{Q}} r F$.

ЗАмЕчАНиЕ 7.9. Справедливо следующее:

$$
r>0 \quad \Longleftrightarrow m>0 \quad \Longleftrightarrow \quad \widetilde{L} \in \overline{\mathbb{C S}}\left(V, \frac{1}{n} \mathscr{D}_{V}\right)
$$

Лемма 7.10. Справедливо $\mathbb{C} S\left(X, \mu \mathscr{D}_{X}\right) \neq \varnothing$.

ДокаЗАТЕльство. Пусть $\mathbb{C} S\left(X, \mu_{X}\right)=\varnothing$. В случае $r>0$ лог-пара $\left(X, \varepsilon \mathscr{D}_{X}\right)$ - каноническая модель для некоторого $\varepsilon \in \mathbb{Q}_{>\mu}$, но $\varkappa(X, \varepsilon \mathscr{D} X) \leqslant 1$ по построению линейной системы $\mathscr{D}_{V}$. В случае $r=0$ лог-пара $\left(V, \frac{1}{n} \mathscr{D}_{V}\right)$ полутерминальна, что противоречит утверждению леммы 7.1 .

Лемма 7.11. Пусть $\mathbb{C} S\left(X, \mathscr{D}_{X}\right)$ содержит кривую $C$ на многообразии $X$, которая содержится в слоях расслоения $\tau$. Тогда лог-пара $\left(X, \mu_{X}\right)$ имеет канонические особенности в общей точке кривой $C$.

ДокАЗАТЕльство. См. доказательство леммы 5.11.

Лемма 7.12. Существует такая композиция б инволючий Бертини общего слоя расслоения $\tau$, что лог-пара $\left(X, \mu_{\sigma} \sigma\left(\mathscr{D}_{X}\right)\right)$ имеет канонические особенности в общих точках кривых на $X$, где $\mu_{\sigma} \in \mathbb{Q}_{>0}$ задано соотношением $K_{X}+\mu_{\sigma} \sigma\left(\mathscr{D}_{X}\right) \sim_{\mathbb{Q}} r_{\sigma} F$ для некоторого неотрицательного рационального числа $r_{\sigma}$.

ДокАЗАТЕЛЬСтво. См. доказательство леммы 5.12.

Заменяя $\beta$ на $\beta \circ \sigma^{-1}$, можно считать, что лог-пара $\left(X, \mu \mathscr{D}_{X}\right)$ имеет канонические особенности в общих точках кривых на трехмерном многообразии $X$. 
Лемма 7.13. Множество $\overline{\mathbb{C S}}\left(V, \frac{1}{n} \mathscr{D}_{V}\right)$ содержит некоторую кривую на многообразии $V$.

ДоКАЗАТЕЛЬСТво. Предположим, что множество $\overline{\mathbb{C S}}\left(V, \frac{1}{n} \mathscr{D}_{V}\right)$ не содержит кривых на $V$. В частности, оно не содержит $\widetilde{L}$. По лемме 7.8 множество $\overline{\mathbb{C S}}\left(V, \frac{1}{n} \mathscr{D} V\right)$ содержит некоторую точку $O_{i}$. Можно считать, что $i=1$. Из леммы 7.10 следует, что множество $\mathbb{C} S\left(X, \frac{1}{n} \mathscr{D}_{X}\right)$ содержит либо некоторую точку $P \in Z_{1}$, либо компоненту приводимой кривой $Z_{1}=Z_{1}^{0} \cup Z_{1}^{1}$, где $Z_{1}$ - приводимый слой бирационального морфизма $f$ над точкой $O_{1}$. Пусть $D_{1}$ и $D_{2}$ - общие поверхности в $\mathscr{D}_{X}$. Тогда

$n^{2}\left(Z_{1}+2 C\right) \equiv n^{2} K_{X}^{2} \equiv D_{1} \cdot D_{2}=\operatorname{mult}_{Z_{1}^{0}}\left(\mathscr{D}_{X}^{2}\right) Z_{1}^{0}+$ mult $_{Z_{1}^{1}}\left(\mathscr{D}_{X}^{2}\right) Z_{1}^{1}+C_{X}+R_{X}$,

где $C$ - кривая в слоях расслоения $\tau$ с $-K_{X} \cdot C=1, C_{X}$-эффективный одномерный цикл на $X$, чьи компоненты содержатся в слоях расслоения $\tau$, а $R_{X}$ - эффективный одномерный цикл на $X$, чьи компоненты не содержатся в слоях расслоения $\tau$.

Предположим, что $\mathbb{C S}\left(X, \frac{1}{n} \mathscr{D}_{X}\right)$ содержит точку $P \in Z_{1}$. Из теоремы 3.12 следует неравенство mult $_{P}\left(D_{1} \cdot D_{2}\right) \geqslant 4 n^{2}$. С другой стороны, $Z_{1}^{0} \cdot F=Z_{1}^{1} \cdot F=2$ и

$$
2 n^{2}=D_{1} \cdot D_{2} \cdot F=\operatorname{mult}_{Z_{1}^{0}}\left(\mathscr{D}_{X}^{2}\right)+\operatorname{mult}_{Z_{1}^{1}}\left(\mathscr{D}_{X}^{2}\right)+R_{X} \cdot F,
$$

где $F$ - слой расслоения $\tau$. В частности, $\operatorname{mult}_{Z_{1}^{0}}\left(\mathscr{D}_{X}^{2}\right)+\operatorname{mult}_{Z_{1}^{1}}\left(\mathscr{D}_{X}^{2}\right) \leqslant 2 n^{2}$ и равенство влечет $R_{X}=\varnothing$. Пусть $H_{Z_{1}}$ - общая поверхность в линейной системе $\left|-K_{X}\right|$, которая содержит приводимую кривую $Z_{1}$. Тогда поверхность $H_{Z_{1}}$ не содержит неприводимых компонент одномерных циклов $C_{X}$ и $R_{X}$. Следовательно, вьполнены неравенства

$$
\begin{aligned}
H_{Z_{1}} \cdot\left(C_{X}+R_{X}\right) & \geqslant \operatorname{mult}_{P}\left(C_{X}\right)+\operatorname{mult}_{P}\left(R_{X}\right) \\
& \geqslant 4 n^{2}-\operatorname{mult}_{Z_{i}^{0}}\left(\mathscr{D}_{X}^{2}\right) \delta_{P}^{0}+\operatorname{mult}_{Z_{1}^{1}}\left(\mathscr{D}_{X}^{2}\right) \delta_{P}^{1},
\end{aligned}
$$

где $\delta_{P}^{i}=\operatorname{mult}_{P}\left(Z_{1}^{i}\right)$. Однако $H_{Z_{1}} \cdot\left(C_{X}+R_{X}\right)=2 n^{2}$. Значит, цикл $R_{X}$ пуст, вьполнено mult $_{P}\left(C_{X}\right)=H_{Z_{1}} \cdot C_{X}=2 n^{2}$ и либо $P=Z_{1}^{0} \cap Z_{1}^{1}$ и выполнено равенство

$$
\text { mult }_{Z_{1}^{0}}\left(\mathscr{D}_{X}^{2}\right)+\text { mult }_{Z_{1}^{1}}\left(\mathscr{D}_{X}^{2}\right)=2 n^{2}
$$

либо $P \in Z_{1}^{k}$, mult $Z_{1}^{k}\left(\mathscr{D}_{X}^{2}\right)=2 n^{2}$ и mult $Z_{1}^{1-k}\left(\mathscr{D}_{X}^{2}\right)=0$. С другой стороны, в последнем случае из $D_{1} \cdot Z_{1-k}^{0}=0$ следует mult $Z_{1}^{1-k}\left(\mathscr{D}_{X}^{2}\right)>0$. Таким образом, $P=Z_{1}^{0} \cap Z_{1}^{1}$ и

$$
\text { mult }_{Z_{1}^{0}}\left(\mathscr{D}_{X}^{2}\right)+\text { mult }_{Z_{1}^{1}}\left(\mathscr{D}_{X}^{2}\right)=2 n^{2}
$$

но из равенства mult ${ }_{P}\left(C_{X}\right)=H_{Z_{1}} \cdot C_{X}$ следует, что все неприводимые компоненты одномерного цикла $C_{X}$ содержатся в слое $F_{P}$ расслоения $\tau$, который проходит через точку $P$. Более того, $\operatorname{mult}_{P}\left(\mathscr{D}_{X}\right)=2 n$ по теореме 3.12 . Зададим $V$ уравнением

$$
u^{2}=x^{2} \sum_{i=0}^{4} \bar{p}_{i}(x, y, z) t^{4-i}+y^{2} \sum_{i=0}^{4} \bar{q}_{i}(x, y, z) t^{4-i}
$$


во взвешенном проективном пространстве $\mathbb{P}(1,1,1,3)$, где $\bar{p}_{i}$ и $\bar{q}_{i}$ - однородные многочлены степени $i, x, y, z$ и $t$ - однородные координаты веса один, а $u$ - однородная координата веса три. Можно считать, что кривая $\widetilde{L}$ задана уравнениями $x=y=0$, а точка $O_{1}$ задана уравнениями $x=y=z=0$. В этом случае либо $\bar{q}_{0}=0$, либо $\bar{p}_{0}=0$ по определению точки $O_{1}$ и по предположению, что существует ровно восемь различных точек $O_{i}$. Без ограничения общности можно считать, что $\bar{q}_{0}=0$. Тогда линейная форма $\bar{q}_{1}(x, y, z)$ не равна тождественно нулю из-за гладкости многообразия $X$. Более того, даже линейная форма $\bar{q}_{1}(0,0, z)$ не равна тождественно нулю в силу первоначального предположения о гладкости исключительного дивизора $E$. Таким образом, пучок $\mathscr{P}$ в линейной системе $\left|-K_{V}\right|$, заданный уравнением $A x+B \bar{q}_{1}(x, y, z)=0$, не имеет базисных компонент, где $A$ и $B$ - комплексные чис-

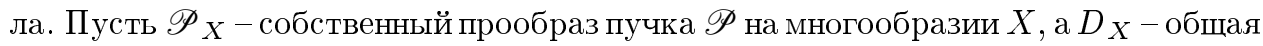
поверхность в пучке $\mathscr{P}_{X}$. Тогда

$$
\operatorname{mult}_{P}\left(D_{X}\right)=2, \quad D_{X} \sim-K_{X}, \quad \operatorname{mult}_{P}\left(D_{1} \cdot D_{X}\right)=4 n
$$

и все вычисления, примененные к одномерному эффективному циклу $D_{1} \cdot D_{2}$, применимы к циклу $D_{1} \cdot D_{X}$. В частности, $D_{1} \cdot D_{X} \subset E \cup F_{P}$. Пусть $O \notin E \cup F_{P}-$ достаточно общая точка на поверхности $D_{1}$, а $D_{O}$ - поверхность в пучке $\mathscr{P}_{X}$, проходящая через точку $O$. Тогда предыдущие аргументы влекут $D_{1}=D_{O}$, поскольку обе поверхности неприводимы. Следовательно, $\mathscr{D}_{V}=\mathscr{P}$, но множество $\overline{\mathbb{C}}(V, \mathscr{P})$ содержит кривые на многообразии $V$, что противоречит сделанному предположению.

Таким образом, множество $\mathbb{C} S\left(X, \frac{1}{n} \mathscr{D}_{X}\right)$ не содержит точек на $X$, но содержит одну или обе из кривых $Z_{1}^{0}$ и $Z_{1}^{1}$. В каждом случае дословное повторение соответствующей части доказательства леммы 7.8 или 5.13 приводит к противоречию.

ЗАМЕчАНИЕ 7.14. Можно считать, что $r>0$.

Пусть $h: U \rightarrow X-$ бирациональный морфизм, $U$ неособо, $\beta \circ f \circ h$ регулярно,

$$
K_{U}+\mu \mathscr{D}_{U} \sim_{\mathbb{Q}} h^{*}(r F)+\sum_{i=1}^{k} a_{i} E_{i},
$$

где $\mathscr{D}_{U}=h^{-1}\left(\mathscr{D}_{X}\right), \quad E_{i}-h$-исключительный дивизор и $a_{i} \in \mathbb{Q}$. Определим подмножество $\mathscr{J} \subset \mathbb{P}^{1}$ как образ исключительных дивизоров $E_{i}$ с $a_{i}<0$. Для $\lambda \in \mathscr{J}$ положим

$$
h^{*}\left(F_{\lambda}\right) \sim h^{-1}\left(F_{\lambda}\right)+\sum_{j=1}^{k_{\lambda}} b_{j} E_{j},
$$

где $F_{\lambda}$ - слой расслоения $\tau$ над точкой $\lambda$ и $b_{i} \in \mathbb{N}$. Наконец, положим $\mathscr{I}=$ $\bigcup_{\lambda \in \mathscr{J}} \mathscr{I}_{\lambda}$, а для $\lambda \in \mathscr{J}$ определим множество $\mathscr{I}_{\lambda} \subset\{1, \ldots, k\}$ следующим образом: $i \in \mathscr{I}_{\lambda}$ в том и только том случае, если $h\left(E_{i}\right)$ является точкой в слое $F_{\lambda}$ и $a_{i}<0$.

УТВЕРЖДЕНИЕ 7.15. Справедливо неравенство

$$
r+\sum_{\lambda \in \mathscr{J}} \min \left\{\frac{a_{i}}{b_{i}} \mid h\left(E_{i}\right) \in F_{\lambda} \quad u \quad a_{i}<0\right\} \leqslant 0 .
$$


ДокаЗАТЕЛЬСтво. Пусть утверждение неверно. Тогда сушествуют $\varepsilon$ и $c_{\lambda}$ в $\mathbb{Q}_{>0}$ такие, что $r=\varepsilon+\sum_{\lambda \in \mathscr{J}} c_{\lambda}$ и $c_{\lambda}+\min \left\{a_{i} / b_{i} \mid h\left(E_{i}\right) \in F_{\lambda}\right.$ и $\left.a_{i}<0\right\}>0$. В частности,

$$
K_{U}+\mu \mathscr{D}_{U} \sim_{\mathbb{Q}} h^{*}(\varepsilon F)+\sum_{\lambda \in \mathscr{J}}\left(h^{*}\left(c_{\lambda} F_{\lambda}\right)+\sum_{i \in \mathscr{I}_{\lambda}} a_{i} E_{i}\right)+\sum_{i \notin \mathscr{I}} a_{i} E_{i},
$$

но для всех $\lambda \in \mathscr{J}$ дивизор $h^{*}\left(c_{\lambda} F_{\lambda}\right)+\sum_{i \in \mathscr{I}_{\lambda}} a_{i} E_{i}$ эффективен в силу выбора положительного рационального числа $c_{\lambda}$, а дивизор $\sum_{i \notin \mathscr{I}} a_{i} E_{i}$ эффективен, поскольку по предположению лог-пара $\left(X, \mu \mathscr{D}_{X}\right)$ имеет канонические особенности в общих точках кривых на многообразии $X$. Пусть $O$ - достаточно общая точка на достаточно обшем слое $D_{O}$ морфизма $\rho \circ f \circ h$, а $C$ - собственный прообраз на $U$ достаточно обшей неприводимой кривой, содержашейся в достаточно обшем слое расслоения $\pi$, такой, что кривая $C$ содержит $O$. Тогда $K_{U} \cdot C=0$ и $\mathscr{D}_{U} \cdot C=0$. Таким образом,

$\left(K_{U}+\mu \mathscr{D}_{U}\right) \cdot C=h^{*}(\varepsilon F) \cdot C+\sum_{\lambda \in \mathscr{J}}\left(h^{*}\left(c_{\lambda} F_{\lambda}\right)+\sum_{i \in \mathscr{I}_{\lambda}} a_{i} E_{i}\right) \cdot C+\sum_{i \notin \mathscr{I}} a_{i} E_{i} \cdot C=0$

и, в частности, $h^{*}(\varepsilon F) \cdot C=0$ ввиду обшности $C$. Пусть $F_{C}$ - слой морфизма $\tau \circ h$, содержащий $O$. Тогда из $h^{*}(F) \cdot C=0$ следует $C \subset F_{C}$, но $D_{O}$ и $F_{C}$ неприводимы ввиду обшности точки $O$. Из обшности $C$ следует $F_{C}=D_{O}$, что невозможно.

Пусть $Z$ - слой морфизма $\left.f\right|_{E}: E \rightarrow \widetilde{L}, C$ - кривая в слоях $\tau$ такая, что $-K_{X} \cdot C=1$, возьмем две обшие поверхности $D_{1}$ и $D_{2}$ в $\mathscr{D}_{X}$. Тогда $\overline{\mathbb{N E}}(X)=$ $\mathbb{R}_{\geqslant 0} Z \oplus \mathbb{R}_{\geqslant 0} C$ и

$$
T_{0}=\mu^{2} D_{1} \cdot D_{2}=Z_{X}+\sum_{\lambda \in \mathbb{P}^{1}} C_{\lambda} \equiv Z+(2+4 r) C
$$

где все неприводимые компоненты одномерного эффективного цикла $Z_{X}$ не содержатся в слоях расслоения $\tau$, а все неприводимые компоненты одномерного эффективного цикла $C_{\lambda}$ содержатся в слое $F_{\lambda}$ расслоения $\tau$ над точкой $\lambda \in \mathbb{P}^{1}$. Таким образом,

$$
-K_{X} \cdot \sum_{\lambda \in \mathbb{P}^{1}} C_{\lambda}=2+4 r \leqslant 2-4 \sum_{\lambda \in \mathscr{J}} \min \left\{\frac{a_{i}}{b_{i}} \mid h\left(E_{i}\right) \in F_{\lambda} \text { и } a_{i}<0\right\}
$$

и, следовательно, существуют точка $\omega \in \mathbb{P}^{1}$ и $h$-исключительный дивизор $E_{t}$ такие, что $h\left(E_{t}\right)$ является точкой $O$ в слое $F_{\omega}$ расслоения $\tau$ над $\omega$, выполнено неравенство

$$
-K_{X} \cdot C_{\omega} \leqslant 2-4 \frac{a_{t}}{b_{t}}
$$

где $a_{t} / b_{t}=\min \left\{a_{i} / b_{i} \mid h\left(E_{i}\right) \in F_{\omega}\right.$ и $\left.a_{i}<0\right\}$. В частности, можно дословно повторить все логические импликации, содержашиеся в $\S 5$, начиная с леммы 5.22 , кроме 
последней, поскольку в данном случае $\mathscr{D}_{X}$ является пучком. Значит, в обозначениях $\S 5$

$$
\begin{gathered}
T_{0}=Z_{X}+C_{\omega}, \quad a\left(X, \mu \mathscr{D}_{X}, G_{N}\right)=-\Sigma_{1}, \quad \Sigma_{0}=\Sigma_{0}^{\prime} \\
b\left(X, F_{\omega}, G_{N}\right)=\Sigma_{0}, \quad r=\frac{\Sigma_{1}}{4 \Sigma_{0}}, \quad \nu_{j}=2
\end{gathered}
$$

носитель цикла $Z_{X}$ содержится в приводимом слое $Z_{i}=Z_{i}^{0} \cup Z_{i}^{1}$ морфизма $f$ и

$$
\begin{gathered}
\operatorname{mult}_{O}\left(Z_{X}\right)=2 n^{2}, \quad \operatorname{mult}_{O}\left(C_{\omega}\right)=2+4 r \\
-K_{X} \cdot C_{\omega}=\operatorname{mult}_{O}\left(C_{\omega}\right), \quad m_{T_{0}}^{j}=m_{T_{0}}^{0}
\end{gathered}
$$

ЗАмечАниЕ 7.16. Слой $F_{\omega}$ является двойным накрытием $\gamma \circ f\left(F_{\omega}\right) \cong \mathbb{P}^{2}$, разветвленным в кривой (возможно особой) степени четыре. Из неособости $F_{\omega}$ в $O$ и равенства $-K_{X} \cdot C_{\omega}=\operatorname{mult}_{O}\left(C_{\omega}\right)$ следует, что носитель цикла $\gamma \circ f\left(C_{\omega}\right)$ состоит из одной прямой в $\mathbb{P}^{3}$, которая проходит через точку $\gamma\left(O_{i}\right) \in L$.

ЛемМа 7.17. Справедливо равенство $O=Z_{i}^{0} \cap Z_{i}^{1}$.

ДокАЗАТЕльство. Пусть $O \neq Z_{i}^{0} \cap Z_{i}^{1}$ и $O \in Z_{i}^{k}$. Тогда носитель цикла $C_{\omega}$ состоит из гладкой рациональной кривой $C \subset X$ такой, что $-K_{X} \cdot C=1$ и $O \in C$. Однако

$$
\left.\left.\mathscr{D}_{X}\right|_{F_{\omega}} \sim(m-n) K_{F_{\omega}} \sim(m-n) K_{X}\right|_{F_{\omega}}
$$

и $\operatorname{mult}_{O}\left(\left.\mathscr{D}_{X}\right|_{F_{\omega}}\right) \geqslant 2(n-m)$. Рассмотрим $F_{\omega}$ как двойное накрытие $\gamma_{F_{\omega}}: F_{\omega} \rightarrow \mathbb{P}^{2}$ с ветвлением в неособой квартике $S_{F_{\omega}} \subset \mathbb{P}^{2}$. В этом случае

$$
\left.\mathscr{D}_{X}\right|_{F_{\omega}}=2(n-m) C
$$

откуда следует, что $\gamma_{F_{\omega}}(C) \subset S_{F_{\omega}}$. Значит, $\gamma_{F_{\omega}}(O) \in S_{F_{\omega}}$ и $O=Z_{i}^{0} \cap Z_{i}^{1}$.

Лемма 7.18. Справедливо равенство $K=1$.

ДоказАтельство. Пусть $K \neq 1$. Тогда $P_{1}$ - точка. Из равенства $m_{T_{0}}^{0}=m_{T_{0}}^{1}$ следует, что точка $P_{1}$ содержится в собственном прообразе кривой $Z_{i}^{k}$ на $X_{1}$, а носитель цикла $Z_{X}$ состоит из $Z_{i}^{k}$. С другой стороны, кривая $Z_{i}^{k}$ пересекает любую кривую в носителе цикла $C_{\omega}$ трансверсально, поскольку поверхность $E$ неособа в $O$ и

$$
E \cdot C_{\omega}=-K_{X} \cdot C_{\omega}=\operatorname{mult}_{O}\left(C_{\omega}\right)
$$

откуда следует, что собственньй прообраз цикла $C_{\omega}$ на многообразии $X_{1}$ не может проходить через точку $P_{1}$. Таким образом, $m_{T_{0}}^{0}>m_{T_{0}}^{1}$. 
ЗАмечание 7.19. Из $\nu_{j}=2$ следует, что граф Г является цепю, $\Sigma_{0}=1$ и $\Sigma_{1}=N-1$.

Ограничение $\mathscr{D}_{X_{1}}$ на $G_{1} \cong \mathbb{P}^{2}$ рационально эквивалентно $\mathscr{O}_{\mathbb{P}^{2}}(2(n-m))$, но выполнено равенство mult $P_{1}\left(\mathscr{D}_{X_{1}}\right)=2(n-m)$. Значит, $\left.\mathscr{D}_{X_{1}}\right|_{G_{1}}=2(n-m) P_{1}$ и $P_{1}$ является прямой на поверхности $G_{1} \cong \mathbb{P}^{2}$. Из равенства $b\left(X, F_{\omega}, G_{N}\right)=\Sigma_{0}$ следует, что $P_{1}$ не лежит на поверхности $F_{\omega}^{1}$. Значит, кратность в точке $P_{1} \cap F_{\omega}^{1}$ общей поверхности в ограничении линейной системы $\mathscr{D}_{X_{1}}$ на поверхность $F_{\omega}^{1}$ не меньше чем $2(n-m)$, откуда следует, что $\left.\mathscr{D}_{X}\right|_{F_{\omega}}=2(n-m) C_{0}$ для гладкой рациональной кривой $C_{0} \subset F_{\omega}$, проходящей через точку $O$, такой, что $-K_{X} \cdot C_{0}=1$. В частности, $\gamma \circ f\left(C_{0}\right)$ является прямой на $S$, которая проходит через $\gamma\left(O_{i}\right)$. Значит, $\gamma \circ f\left(C_{0}\right)=L$ и $C_{0}=F_{\omega} \cap E$, поскольку предполагалось, что $L$ является единственной прямой на секстике $S$, которая проходит через одну из точек $\gamma\left(O_{i}\right) \in \mathbb{P}^{3}$. Однако условие $C_{0}=F_{\omega} \cap E$ противоречит гладкости поверхности $E$. Таким образом, теорема 1.15 доказана полностью.

\section{Список литературы}

1. Kollár J. Rational curves on algebraic varieties. Berlin: Springer-Verlag, 1995.

2. Манин Ю. И. Кубические формы. М.: Наука, 1972.

3. Cheltsov I. A. Log models on birationally rigid varieties // J. Math. Sci. (New York). 2000. V. 102. № 2. P. 3843-3875.

4. Manin Yu. I. Rational surfaces over perfect fields // Inst. Hautes Études Sci. Publ. Math. 1966. V. 30. P. 55-114.

5. Манин Ю.И. Рационалные поверхности над совершенными полями. II // Матем. сб. 1967. T. $72(114)$. C. 161-192.

6. Bogomolov F., Tschinkel Yu. On the density of rational points on elliptic fibrations // J. Reine Angew. Math. 1999. V. 511. P. 87-93.

7. Harris J., Tschinkel Yu. Rational points on quartics // Duke Math. J. 2000. V. 104. P. $477-500$.

8. Ryder D. Elliptic and K3 fibrations birational to Fano 3-fold weighted hypersurfaces // Thesis. Warwick: Univ. Warwick, 2002.

9. Чельцов И. А. Лог-пары на гиперповерхностях степени $N$ в $\mathbb{P}^{N} / /$ Матем. заметки. 2000. Т. 68. №1. С. 131-138.

10. Чельцов И. А. О гладкой четырехмерной квинтике // Матем. сб. 2000. Т. 191.№9. C. $139-162$.

11. Чельцов И. А. Многообразие Фано с единственной эллиптической структурой // Матем. сб. 2001. Т. 192. № 5. С. 145-156.

12. Гриненко М. М. Бирациональные свойства пучков поверхностей дель Пеццо степени 1 и $2 / /$ Матем. сб. 2000. Т. 191. № 5. С. 17-38.

13. Grinenko M. On the birational rigidity of some pencils of del Pezzo surfaces // J. Math. Sci. (New York). 2000. V. 102. № 2. P. 3933-3937.

14. Гриненко М. М. О послойных перестройках расслоений на поверхности дель Пеццо степени 2 // УМН. 2001. Т. 56. № 4. С. 145-146.

15. Пухликов $A$. В. Бирациональные автоморфизмы трехмерных алгебраических многообразий с пучком поверхностей дель Пещц // Изв. РАН. Сер. матем. 1998. Т. 62. №1. C. $123-164$.

16. Alexeev V. General elephants of $\mathbb{Q}$-Fano 3-folds // Compositio Math. 1994. V. 91. P. 91-116.

17. Kawamata Y., Matsuda K., Matsuki K. Introduction to the minimal model problem // Adv. Stud. Pure Math. 1987. V. 10. P. 283-360. 
18. Kollár J. (ed.) Flips and abundance for algebraic threefolds. A summer seminar at the University of Utah, Salt Lake City, 1991. Paris: Soc. Math. France, 1992. (Astérisque. V. 211.)

19. Corti A. Singularities of linear systems and 3-fold birational geometry // Explicit birational geometry of 3-folds / ed. A. Corti et al. Cambridge: Cambridge Univ. Press, 2000. P. 259-312. (London Math. Soc. Lecture Note Ser. V. 281.)

20. Pukhlikov A. V. Essentials of the method of maximal singularities // Explicit birational geometry of 3-folds / ed. A. Corti et al. Cambridge: Cambridge Univ. Press, 2000. P. 73-100. (London Math. Soc. Lecture Note Ser. V. 281.)

21. Corti A. Factorizing birational maps of threefolds after Sarkisov // J. Algebraic Geom. 1995. V. 4. P. 223-254.

22. Kawakita $M$. Divisorial contractions in dimension 3 which contracts divisors to smooth points // Invent. Math. 2001. V. 145. P. 105-119.

Математический институт Макса Планка,

Поступила в редакцию

г. Бонн, Германия

07.10 .2003

E-mail: cheltsov@yahoo.com 